\title{
ON CLASSIFICATION OF LORENTZIAN KAC-MOODY ALGEBRAS
}

\author{
VAlery A. Gritsenko and ViacheslaV V. Nikulin ${ }^{1}$
}

\begin{abstract}
We discuss a general theory of Lorentzian Kac-Moody algebras which should be a hyperbolic analogy of the classical theories of finite-dimensional semisimple and affine Kac-Moody algebras. First examples of Lorentzian Kac-Moody algebras were found by Borcherds. We consider general finiteness results about the set of Lorentzian Kac-Moody algebras of the rank $\geq 3$, and the problem of their classification. As an example, we give classification of Lorentzian Kac-Moody algebras of the rank three with the hyperbolic root lattice $S_{t}^{*}$, symmetry lattice $L_{t}^{*}$, and the symmetry group $\widehat{O}^{+}\left(L_{t}\right), t \in \mathbb{N}$, where
\end{abstract}

$$
H=\left(\begin{array}{cc}
0 & -1 \\
-1 & 0
\end{array}\right), S_{t}=H \oplus\langle 2 t\rangle=\left(\begin{array}{ccc}
0 & 0 & -1 \\
0 & 2 t & 0 \\
-1 & 0 & 0
\end{array}\right), L_{t}=H \oplus S_{t}=\left(\begin{array}{ccccc}
0 & 0 & 0 & 0 & -1 \\
0 & 0 & 0 & -1 & 0 \\
0 & 0 & 2 t & 0 & 0 \\
0 & -1 & 0 & 0 & 0 \\
-1 & 0 & 0 & 0 & 0
\end{array}\right),
$$

and $\widehat{O}^{+}\left(L_{t}\right)=\left\{g \in O^{+}\left(L_{t}\right) \mid g\right.$ is trivial on $\left.L_{t}^{*} / L_{t}\right\}$ is an extended paramodular group. Perhaps, this is the first example when a large class of Lorentzian Kac-Moody algebras was classified.

\section{INTRODUCTION}

There are two well-known and very important in Mathematics and Physics types of Lie algebras. The first is the class of finite-dimensional semi-simple (or just finite) Lie algebras. The second is the class of affine Kac-Moody algebras. Both these classes belong to the general class of Kac-Moody Lie algebras. See [47] about their theory.

After fundamental results by $\mathrm{R}$. Borcherds [2] — [7], it seems, we now know a right definition of the next type of Lie algebras which should serve as a hyperbolic analogy of finite (or elliptic type) and affine (or parabolic type) Lie algebras. Here we call this type of Lie algebras as Lorentzian Kac-Moody algebras, but one may prefer a different name, e. g. hyperbolic of Borcherds type. Algebras of this type were very important for Borcherds solution [5] of the famous Moonshine Conjecture of Conway and Norton [20] about modular properties of representations of the sporadic finite simple group with the name Monster.

Main features of the theory of Lorentzian Kac-Moody algebras $\mathfrak{g}$ are as follows (see details of this definition in Sect. 1.4):

1) A Lorentzian Kac-Moody algebra $\mathfrak{g}$ is graded

$$
\mathfrak{g}=\bigoplus_{\alpha \in R} \mathfrak{g}_{\alpha}=\mathfrak{g}_{0} \bigoplus\left(\bigoplus_{\alpha \in \Delta_{+}} \mathfrak{g}_{\alpha}\right) \bigoplus\left(\bigoplus_{\alpha \in \Delta_{+}} \mathfrak{g}_{-\alpha}\right)
$$

\footnotetext{
${ }^{1}$ Supported by Russian Fund of Fundamental Research (grant 00-01-00170).
} 
by a hyperbolic root lattice $R$ which is a free $\mathbb{Z}$-module of a finite rank equipped with an integral symmetric bilinear form which is non-degenerate and has exactly one negative square. For finite and affine Kac-Moody algebras the root lattice $R$ is positive definite and semi-positive definite respectively.

2) Weyl group $W \subset O(R)$ is a reflection group in the hyperbolic space (or a Lobachevsky space) $\mathcal{L}(R)$ related with $R$. For finite and affine Kac-Moody algebras the Weyl group is a finite reflection group and a discrete reflection group in Euclidean space respectively.

3) The real part of the root system of the algebra $\mathfrak{g}$ which is some subset of roots in the root lattice $R$, is some hyperbolic analogy of finite and affine root systems. Like for finite and affine root systems, it is defined by a fundamental chamber $\mathcal{M} \subset \mathcal{L}(R)$ of $W$ and the set $P(\mathcal{M})$ of roots of $R$ which are orthogonal to faces of $\mathcal{M}$ of highest dimension $(P(\mathcal{M})$ is the set of simple real roots of the algebra $\mathfrak{g})$.

4) Denominator identity of $\mathfrak{g}$ (due to Weyl, Kac and Borcherds) defines an automorphic form $\Phi$ on a IV type (in classification of É. Cartan) Hermitian symmetric domain related with $R$ which should agree with the Weyl group $W$. The denominator identity has the form

$$
\begin{aligned}
& \Phi=\exp (-2 \pi i(\rho, z)) \prod_{\alpha \in \Delta_{+}}(1-\exp (-2 \pi i(\alpha, z)))^{\operatorname{mult}(\alpha)}= \\
= & \sum_{w \in W} \varepsilon(w)\left(\exp (-2 \pi i(w(\rho), z))-\sum_{a \in S \cap \mathbb{R}_{++} \mathcal{M}} m(a) \exp (-2 \pi i(w(\rho+a), z))\right) .
\end{aligned}
$$

Its infinite product part is the infinite product expansion of the automorphic form $\Phi$ where multiplicities mult $(\alpha)$ give dimensions of the root spaces $\mathfrak{g}_{\alpha}$ of $\mathfrak{g}$. Its infinite sum part is the Fourier expansion of the automorphic form $\Phi$ which defines the algebra $\mathfrak{g}$ by generators and defining relations similar to of Killing, Cartan, Chevalley and Serre. The element $\rho \in R \otimes \mathbb{Q}$ is the Weyl vector defined by the equality

$$
(\rho, \alpha)=-\alpha^{2} / 2 \text { for any } \alpha \in P(\mathcal{M}) \text {. }
$$

For finite and affine Kac-Moody algebras the denominator identity gives a polynomial and a Jacobi modular form respectively. We want to keep this modularity property for Lorentzian Kac-Moody algebras.

5) The automorphic form $\Phi$ should be reflective which means that the divisor of $\Phi$ should be union of rational quadratic divisors orthogonal to roots. One can consider this condition as the globalization on the whole Hermitian symmetric domain of zeros of the infinite product expansion of $\Phi$ in the neighborhood of the cusp at infinity of the Hermitian symmetric domain where the infinite product converges. This reflectivity property is valid in all known interesting cases, and it seems, it distinguishes the most interesting Lie algebras. Moreover, this additional condition is very important for classification.

For hyperbolic case, one cannot satisfy conditions 1) - 4) inside usual class of Kac-Moody algebras. Fundamental discovery of Borcherds is that one can satisfy conditions 1) — 5) after an appropriate generalization of Kac-Moody algebras to so called generalized Kac-Moody algebras. He constructed many examples of generalized Kac-Moody algebras satisfying the conditions 1) - 5) above. See [2] - [7]. We give the most multi-dimensional and interesting Borcherds example in 
will be generalized Kac-Moody algebras or superalgebras satisfying conditions 1) 5). See details of the definition in Sect. 1.4.

Lorentzian Kac-Moody algebras used by Borcherds for solution of Moonshine Conjecture are graded by the unimodular hyperbolic plane (of the rank two) $H=$ $\left(\begin{array}{cc}0 & -1 \\ -1 & 0\end{array}\right)$, and their denominator identity is an automorphic form $j_{g}\left(\tau_{1}\right)-j_{g}\left(\tau_{2}\right)$, $\tau_{1}, \tau_{2} \in \mathbb{H}$, on the product $\mathbb{H} \times \mathbb{H}$ of two upper-half planes $\mathbb{H}$ where $g$ is a conjugacy class of the Monster and $j_{g}(\tau)$ is an appropriate normalized Hauptmodul. There are many papers and reviews written on the subject of Borcherds solution [5] of Moonshine Conjecture. E. g. see [6], [10], [27], [70]. We don't consider that in the paper. We also don't consider relation of Lorentzian Kac-Moody algebras with Vertex Algebras which is very important for solution of Moonshine Conjecture. E.g. see [2] — [6], [11], [25], [27], [28], [49] about this subject.

In this paper we consider classification problem of Lorentzian Kac-Moody algebras of the rank rk $R \geq 3$. One of the main properties of finite and affine Kac-Moody algebras is that they are classified (by Killing). Finite Kac-Moody algebras are classified by Dynkin diagrams, and affine Kac-Moody algebras are classified by extended Dynkin diagrams. In this paper we consider similar property of Lorentzian Kac-Moody algebras of the rank rk $R \geq 3$. We consider results and conjectures which show that number of Lorentzian Kac-Moody algebras of the rank $\geq 3$ is in essential finite. Thus, Lorentzian Kac-Moody algebras of the rank $\geq 3$ are all exceptional (like exceptional Lie algebras of the type $\mathbb{E}_{n}, n=6,7,8, \mathbb{F}_{4}$, $\mathbb{G}_{2}$ ) and can be classified in principle. We mention that for the ranks one and two analogous classification problems are much simpler, but we expect that number of cases is in essential infinite for this case.

In $\S 1$ we consider general definitions and theory of Lorentzian Kac-Moody algebras (Sects. 1.1 - 1.4), and general finiteness results and conjectures about Lorentzian Kac-Moody algebras of the rank $\geq 3$ (Sects. 1.5 - 1.7). Main result here is that number of data 1) - 3) in data 1) - 4) is finite (or is in essential finite) for $\mathrm{rk} R \geq 3$. We also give some classification results about data 1) - 3) for the rank three case. All these results are closely related with the old results of the second author and Vinberg about finiteness of the set of arithmetic reflection groups in hyperbolic spaces. See [58] - [61], [71] - [73].

The main property of the data 1) - 3) in data 1) - 4) which permits to get these results, is that the fundamental chamber $\mathcal{M}$ of the Weyl group $W$ has finite or almost finite volume. Exactly here one uses that the denominator identity in 4) gives an automorphic form. More exactly, let $\operatorname{Sym}(\mathcal{M}) \subset O^{+}(R)$ be the symmetry group of $\mathcal{M}$. Then the corresponding semi-direct product $W \rtimes \operatorname{Sym}(\mathcal{M})$ has finite index in $O^{+}(R)$, and there exists a non-zero $\rho \in R \otimes \mathbb{Q}$ (it is called a generalized Weyl vector) such that the orbit $\operatorname{Sym}(\mathcal{M})(\rho)$ is finite. Hyperbolic lattices $R$ having a reflection subgroup $W \subset O(R)$ with this property are called reflective. Since $O^{+}(R)$ is arithmetic and has a fundamental domain of finite volume, it follows that $\mathcal{M}$ is finite of finite volume if $\rho^{2}<0$ (elliptic type); $\mathcal{M}$ is finite of finite volume in any angle with the vertex at infinity $\mathbb{R}_{++} \rho$ if $\rho^{2}=0$ (parabolic type); $\mathcal{M}$ is finite of finite volume in any orthogonal cylinder over compact set in the hyperplane orthogonal to $\rho$ if $\rho^{2}>0$ (hyperbolic type). Using this property of the fundamental chamber $\mathcal{M}$, one can prove that the number of reflective hyperbolic lattices $R$ is finite for rk $R \geq 3$. See [57] — [61], [63], [64], [66], [68], [71]—[73]. Existence of the Weyl 
(i.e. finiteness up to a very simple equivalence relation) of data 1) - 3) in data 1) $-4)$.

In Sect. 1.6 we consider finiteness conjectures and results about data 4) and $5)$. Some observations from [65] and [40] give a hope that number of these data is also finite for $\mathrm{rk} R \geq 3$. It seems, some general results about infinite automorphic products and their divisors by Borcherds [7], [9] and by Bruinier [14] - [16] are also related with this subject. We don't discuss these results of Borcherds and Bruinier in this paper.

In Sect. 2 we consider a concrete example of classification of Lorentzian KacMoody algebras of the rank three where we use general ideas and results from $\S 1$. We give classification of Lorentzian Kac-Moody algebras with the root lattice $S_{t}^{*}$ where

$$
S_{t}=H \oplus\langle 2 t\rangle=\left(\begin{array}{ccc}
0 & 0 & -1 \\
0 & 2 t & 0 \\
-1 & 0 & 0
\end{array}\right),
$$

the symmetry lattice $L_{t}^{*}$ where

$$
L_{t}=H \oplus S_{t}=\left(\begin{array}{ccccc}
0 & 0 & 0 & 0 & -1 \\
0 & 0 & 0 & -1 & 0 \\
0 & 0 & 2 t & 0 & 0 \\
0 & -1 & 0 & 0 & 0 \\
-1 & 0 & 0 & 0 & 0
\end{array}\right)
$$

and the symmetry group (of the automorphic form $\Phi$ )

$$
\widehat{O}^{+}\left(L_{t}\right)=\left\{g \in O^{+}\left(L_{t}\right) \mid g \text { is trivial on } L_{t}^{*} / L_{t}\right\} .
$$

Here $t \in \mathbb{N}$, and $\oplus$ denotes the orthogonal sum of lattices, and $*$ denotes the dual lattice. We prove (Theorem 2.1.1) that there are exactly 29 Lorentzian KacMoody algebras $\mathfrak{g}$ (or data 1$)-5$ )) with the root lattice $S_{t}^{*}$, symmetry lattice $L_{t}^{*}$ and the symmetry group $\widehat{O}^{+}\left(L_{t}\right)$. It seems, this result is the first where a large class of Lorentzian Kac-Moody algebras was classified. $\S 2$ is devoted to the outline of the proof of this result. Actually, we prove much more general classification result about reflective automorphic forms with the root lattice $S_{t}^{*}$, the symmetry lattice $L_{t}^{*}$, the symmetry group $\widehat{O}^{+}\left(L_{t}\right)$ and an infinite product expansion similar to (0.1) (Theorems 2.2.3 and 2.4.1). This result gives information about Lorentzian Kac-Moody algebras with symmetry lattices $L$ which are equivariant sublattices $L \subset L_{t}$ of the same rank $\operatorname{rk} L=\operatorname{rk} L_{t}=5$. Here equivariant means that $O(L) \subset$ $O\left(L_{t}\right)$. Moreover, one can expect that the corresponding infinite product $=$ infinite sum identities (similar to (0.1)) for these reflective automorphic forms could be related with some interesting algebras similar to generalized Kac-Moody algebras and superalgebras.

In this paper we outline and present all ideas of the proof of the classification Theorem 2.1.1. We hope to present details of the proof and calculations in forthcoming more longer publication.

All 29 Lorentzian Kac-Moody algebras of Theorem 2.1.1 had been constructed in [41]. To construct these algebras, one need to construct the corresponding 29 automorphic forms (0.1). They were constructed in [41] together with their infi- 
of the algebra. We construct all 29 automorphic forms of Theorem 2.1.1 using some variant for Jacobi modular forms of Borcherds exponential lifting. See [41] about this variant. This construction requires delicate calculations with appropriate Jacobi modular forms of two variables with integral Fourier coefficients. We use classification of these forms obtained in [33], [34]. We present some of these results in $\S 3$ : Appendix.

To proof completeness of the list of these 29 automorphic forms, we find (Theorem 2.3.2) all reflective hyperbolic lattices $S_{t}$ of the rank three. Only for them an automorphic form may exist. In particular, $t \leq 105$ for them. When the lattice $S_{t}$ is reflective, we find all possible data 1) - 3) for it and predict the divisor of the reflective automorphic form $\Phi$. Then we can see that one of 29 automorphic forms of Theorem 2.1.1 has the same divisor and coincides with $\Phi$ by Koecher principle.

In general, in [68] all reflective hyperbolic lattices of the rank three were classified: there are 122 main elliptic and 66 main hyperbolic types (there are no main parabolic types). These results and reasonable number of cases give a hope that all Lorentzian Kac-Moody algebras of the rank three will be classified in a future. Finiteness results for rk $R \geq 3$ give a hope that the same can be done for all ranks rk $R \geq 3$. We expect that number of cases drops when the rank is increasing: There are no algebras when rk $R$ is sufficiently high. Borcherds example which we present in Sect. 1.3 is the most highest dimensional known example of Lorentzian Kac-Moody algebras. For this example rk $R=26$.

In this paper, we don't tuch possible Physical applications of Lorentzian KacMoody algebras. One can find some of them in physical papers [17], [18], [21] [23], [28], [38], [42], [45], [51] - [53], [56].

This paper is written during our stay in the University of Lille 1, the University of Liverpool, Steklov Mathematical Institute, Moscow and St. Petersburg, MaxPlanck-Institut für Mathematik, Bonn, and Newton Institute for Mathematical Sciences, Cambridge. We are grateful to the Institutes for hospitality.

\section{A Theory of Lorentzian KaC-Moody Algebras AND GENERAL FINITENESS RESULTS AND CONJECTURES}

We start with a variant of Theory of Lorentzian Kac-Moody algebras which one can consider as a hyperbolic analogy of classical theories of finite and affine Kac-Moody algebras. Here we follow Borcherds and [36], [40], [41], [64], [67].

1.1. Some general results on Kac-Moody algebras. One can find all definitions and details of this section in the classical book by Kac [47].

A generalized Cartan matrix $A$ is an integral square matrix of a finite rank which has only 2 on the diagonal and non-positive integers out of the diagonal. We shall consider only symmetrizable generalized Cartan matrices $A$. It means that there exists a diagonal matrix $D$ with positive rational diagonal coefficients such that $B=D A$ is integral and symmetric. Then $B$ is called the symmetrization of $A$. By definition, $\operatorname{sign}(A)=\operatorname{sign}(B)$. We shall suppose that $A$ is indecomposable which means that there does not exist a decomposition $I=I_{1} \cup I_{2}$ of the set $I$ of indices of $A$ such that $a_{i j}=0$ if $i \in I_{1}$ and $j \in I_{2}$.

Each generalized Cartan matrix $A$ defines a Kac-Moody Lie algebra $\mathfrak{g}(A)$ over $\mathbb{C}$. The Kac-Moody algebra $\mathfrak{g}(A)$ is defined by the set of generators and defining relations prescribed by the generalized Cartan matrix $A$. They are due to V. Kac 
Killing, Cartan, Weyl, Chevalley and Serre about finite-dimensional semi-simple Lie algebras. One should introduce the set of generators $h_{i}, e_{i}, f_{i}, i \in I$, with defining relations

$$
\left\{\begin{array}{l}
{\left[h_{i}, h_{j}\right]=0, \quad\left[e_{i}, f_{i}\right]=h_{i}, \quad\left[e_{i}, f_{j}\right]=0, \text { if } i \neq j,} \\
{\left[h_{i}, e_{j}\right]=a_{i j} e_{j}, \quad\left[h_{i}, f_{j}\right]=-a_{i j} f_{j},} \\
\left(a d e_{i}\right)^{1-a_{i j}} e_{j}=\left(a d f_{i}\right)^{1-a_{i j}} f_{j}=0, \text { if } i \neq j .
\end{array}\right.
$$

The algebra $\mathfrak{g}(A)$ is simple or almost simple: it is simple after factorization by some known central ideal.

We mention some general features of the theory of Kac-Moody algebras $\mathfrak{g}(A)$.

1. The symmetrization $B$ defines a free $\mathbb{Z}$-module $Q=\sum_{i \in I} \mathbb{Z} \alpha_{i}$ with generators $\alpha_{i}, i \in I$, equipped with symmetric bilinear form $\left(\left(\alpha_{i}, \alpha_{j}\right)\right)=B$ defined by the symmetrization $B$. The $Q$ is called root lattice. The algebra $\mathfrak{g}(A)$ is graded by the root lattice $Q$ (by definition, generators $h_{i}, e_{i}, f_{i}$ have weights $0, \alpha_{i},-\alpha_{i}$ respectively):

$$
\mathfrak{g}(A)=\bigoplus_{\alpha \in Q} \mathfrak{g}_{\alpha}=\mathfrak{g}_{0} \bigoplus\left(\bigoplus_{\alpha \in \Delta_{+}} \mathfrak{g}_{\alpha}\right) \bigoplus\left(\bigoplus_{\alpha \in-\Delta_{+}} \mathfrak{g}_{\alpha}\right)
$$

where $\mathfrak{g}_{\alpha}$ are finite dimensional linear spaces, $\left[\mathfrak{g}_{\alpha}, \mathfrak{g}_{\beta}\right] \subset \mathfrak{g}_{\alpha+\beta}, \mathfrak{g}_{0} \equiv Q \otimes \mathbb{C}$ is commutative, and is called Cartan subalgebra. An element $0 \neq \alpha \in Q$ is called root if $\mathfrak{g}_{\alpha} \neq 0$. The $\mathfrak{g}_{\alpha}$ is called root space corresponding to $\alpha$. The dimension $\operatorname{mult}(\alpha)=\operatorname{dim} \mathfrak{g}_{\alpha}$ is called multiplicity of the root $\alpha$. In (1.1.2), $\Delta \subset Q$ is the set of all roots. It is divided in the set of positive $\Delta_{+} \subset \sum_{i \in I} \mathbb{Z}_{+} \alpha_{i}$ and negative $-\Delta_{+}$ roots. A root $\alpha \in \Delta$ is called real if $(\alpha, \alpha)>0$. Otherwise (if $(\alpha, \alpha) \leq 0$ ), it is called imaginary. Every real root $\alpha$ defines a reflection $s_{\alpha}: x \mapsto x-(2(x, \alpha) /(\alpha, \alpha)) \alpha$, $x \in Q$. All reflections $s_{\alpha}$ in real roots generate Weyl group $W \subset O(S)$. The set of roots $\Delta$ and multiplicities of roots are $W$-invariant.

2. One has Weyl-Kac denominator identity which permits to calculate multiplicities of roots:

$$
e(-\rho) \prod_{\alpha \in \Delta_{+}}(1-e(-\alpha))^{\operatorname{mult}(\alpha)}=\sum_{w \in W} \operatorname{det}(w) e(-w(\rho)) .
$$

Here $e(\cdot) \in \mathbb{Z}[Q]$ are formal exponents where $\mathbb{Z}[Q]$ is the group ring of the root lattice $Q$. The $\rho$ is called Weyl vector and is defined by the condition $\left(\rho, \alpha_{i}\right)=$ $-\left(\alpha_{i}, \alpha_{i}\right) / 2$ for any $i \in I$.

The identity (1.1.3) is combinatorial, and direct formulae for multiplicities $\operatorname{mult}(\alpha)$ are unknown in general. One approach to solve this problem is to replace the formal function (1.1.3) by non-formal one (e. g. replacing formal exponents by non-formal ones) to get a function with "good" properties. These good properties may help to find the formulae for multiplicities.

1.2. Finite and affine cases. There are two cases when we have very clear picture (or Theory) of Kac-Moody algebras.

Finite case: The generalized Cartan matrix $A$ is positive definite, $A>0$. Then $\mathfrak{g}(A)$ is finite-dimensional, and we get the classical theory of finite-dimensional 
Affine case: The generalized Cartan matrix $A$ is semi-positive definite, $A \geq 0$. Then $\mathfrak{g}(A)$ is called affine.

For both these cases we have three very nice properties:

(I) There exists classification of all possible generalized Cartan matrices $A$ and the corresponding algebras $\mathfrak{g}(A)$ : They are classified by Dynkin (for finite case) and by extended Dynkin (for affine case) diagrams.

(II) In the denominator identity (1.1.3), formal exponents may be replaces by non-formal ones to give a function with nice properties: For finite case this gives a polynomial. For affine case this gives a Jacobi modular form. Using these properties (or directly), one can find all multiplicities.

(III) Both these cases have extraordinary importance in Mathematics and Physics.

We want to construct similar Theory for Lorentzian (or hyperbolic) case when the generalized Cartan matrix $A$ is hyperbolic: it has exactly one negative square, all its other squares are either positive or zero. There are plenty of hyperbolic generalized Cartan matrices, it is impossible to find all of them and classify. On the other hand, probably not all of them give interesting Kac-Moody algebras, and one has to impose natural conditions on these matrices.

1.3. Lorentzian case. Borcherds example. We have the following key example due to R. Borcherds [3]- $[6]$.

For Borcherds example, the root lattice $Q=S$ where $S$ is a hyperbolic even unimodular lattice $S$ of signature $(25,1)$. Here "even" means that $(x, x)$ is even for any $x \in S$. "Unimodular" means that the dual lattice $S^{*}$ coincides with $S$, equivalently, for a basis $e_{1}, \ldots, e_{26}$ of $S$ the determinant of the Gram matrix $\left(\left(e_{i}, e_{j}\right)\right)$ is equal to \pm 1 . A lattice $S$ with these properties is unique up to isomorphism. For Borcherds example, the Weyl group $W$ is generated by reflections $s_{\alpha}: x \mapsto x-(x, \alpha) \alpha, x \in S$, in all elements $\alpha \in S$ with $\alpha^{2}=2$. The group $W$ is discrete in the hyperbolic space $\mathcal{L}(S)=V^{+}(S) / \mathbb{R}_{++}$where $\mathbb{R}_{+}$and $\mathbb{R}_{++}$denote the sets of non-negative and positive real numbers respectively. Here $V^{+}(S)$ is the positive cone, i. e. a half of the cone $V(S)=\left\{x \in S \otimes \mathbb{R} \mid x^{2}<0\right\}$ of the hyperbolic lattice $S$. The $\mathcal{L}(S)$ is the set of rays in $V^{+}(S)$.

A fundamental chamber $\mathcal{M} \subset \mathcal{L}(S)$ for $W$ is defined by the set $P$ of elements $\alpha \in S$ with $\alpha^{2}=2$ which are orthogonal to $\mathcal{M}$. It has the following description due to Conway [19]. There exists an orthogonal decomposition $S=[\rho, e] \oplus L$ where the Gram matrix of elements $\rho, e$ is equal to $H=\left(\begin{array}{cc}0 & -1 \\ -1 & 0\end{array}\right)$ (in particular, $(\rho, \rho)=0)$, and $L$ is the Leech lattice, i. e. positive definite even unimodular lattice of the rank 24 without elements with square 2 . The set $P$ of roots which are orthogonal to the fundamental chamber $\mathcal{M}$ (or the set of simple real roots) of $W$ is equal to

$$
P=\{\alpha \in S \mid(\alpha, \alpha)=2 \quad \& \quad(\rho, \alpha)=-1\} .
$$

It means that the fundamental chamber $\mathcal{M} \subset \mathcal{L}(S)$ is equal to

$$
\mathcal{M}=\left\{\mathbb{R}_{++} x \in \mathcal{L}(S) \mid(x, P) \leq 0\right\}
$$

and $P$ is a minimal set with this property. We mention that the fundamental chamber $\mathcal{M}$ has "almost finite" volume. It means that $\mathcal{M}$ is finite in any angle 
The matrix

$$
A=\left(\left(\alpha, \alpha^{\prime}\right)\right), \quad \alpha, \alpha^{\prime} \in P
$$

is a generalized Cartan matrix and $\rho$ is the Weyl vector:

$$
(\rho, \alpha)=-(\alpha, \alpha) / 2, \forall \alpha \in P \text {. }
$$

Thus, $A$ defines the Kac-Moody algebra $\mathfrak{g}(A)$ graded by the hyperbolic lattice $S$. But the algebra $\mathfrak{g}(A)$ is not the algebra which is considered for Borcherds example. One has to "correct" the algebra $\mathfrak{g}(A)$.

We have the classical $S L_{2}(\mathbb{Z})$-modular cusp form $\Delta$ of the weight 12 on the upper-half plane $\operatorname{Im} \tau>0$ :

$$
\Delta=q \prod_{n=1}^{\infty}\left(1-q^{n}\right)^{24}=\sum_{m \geq 0} \tau(m) q^{m}
$$

where $q=\exp (2 \pi i \tau)$. We have

$$
\Delta^{-1}=\sum_{n \geq 0} p_{24}(n) q^{n-1}
$$

where $p_{24}(n)$ are positive integers. Borcherds [4] proved the identity

$$
\begin{gathered}
\Phi(z)=\exp (-2 \pi i(\rho, z)) \prod_{\alpha \in \Delta_{+}}(1-\exp (-2 \pi i(\alpha, z)))^{p_{24}(1-(\alpha, \alpha) / 2)}= \\
\sum_{w \in W} \operatorname{det}(w) \sum_{m>0} \tau(m) \exp (-2 \pi i(w(m \rho), z)) .
\end{gathered}
$$

Here $\Delta_{+}=\left\{\alpha \in S \mid \alpha^{2}=2 \quad \& \quad(\alpha, \rho)<0\right\} \cup\left(S \cap \overline{V^{+}(S)}-\{0\}\right)$. The variable $z$ runs through the complexified positive cone $\Omega\left(V^{+}(S)\right)=S \otimes \mathbb{R}+i V^{+}(S)$. Moreover, Borcherds [6], [7] proved that the function $\Phi(z)$ is an automorphic form of weight 12 with respect to the group $O^{+}(T)$ where $T=H \oplus S$ is the extended lattice of the signature $(26,2)$ (we denote as $\oplus$ the orthogonal sum). The group $O^{+}(T)$ naturally acts in the Hermitian symmetric domain of type IV

$$
\Omega(T)=\{\mathbb{C} \omega \subset T \otimes \mathbb{C} \mid(\omega, \omega)=0 \quad \& \quad(\omega, \bar{\omega})<0\}_{0},
$$

which has canonical identification with $\Omega\left(V^{+}(S)\right)$ as follows: $z \in \Omega\left(V^{+}(S)\right)$ defines the element $\mathbb{C} \omega_{z} \in \Omega(T)$ where $\omega_{z}=((z, z) / 2) e_{1}+e_{2} \oplus z \in T \otimes \mathbb{C}$ and $e_{1}, e_{2}$ is the basis of the lattice $H$ with the Gram matrix $H$ above. Here "automorphic of the weight 12 " means that the function $\widetilde{\Phi}\left(\lambda \omega_{z}\right)=\lambda^{-12} \Phi(z), \lambda \in \mathbb{C}^{*}$, is homogeneous of the degree -12 (it is obvious) in the homogeneous cone $\widetilde{\Omega(T)}$ over $\Omega(T)$, and $\widetilde{\Phi}(g \omega)=\operatorname{det}(g) \widetilde{\Phi}(\omega)$ for any $\omega \in \widetilde{\Omega(T)}$ and any $g \in O^{+}(T)$ where $O^{+}(T)$ is the subgroup of index 2 of the group $O(T)$ which keeps the connected component (1.3.8) (marked by 0$)$.

The identity (1.3.7) looks very familiar to the form (1.1.3) of the denominator 
To interpret (1.3.7) as a denominator identity of a Lie algebra, Borcherds introduced [3] generalized Kac-Moody algebras $\mathfrak{g}\left(A^{\prime}\right)$ which correspond to more general matrices $A^{\prime}$ than generalized Cartan matrices. Here we shall call them as generalized Cartan-Borcherds matrices. Difference is that a generalized Cartan-Borcherds matrix $A^{\prime}$ may also have non-positive real elements $a_{i j} \leq 0$ on the diagonal and out of the diagonal, but all $a_{i j} \in \mathbb{Z}$ if $a_{i i}=2$. A definition of the generalized KacMoody algebra $\mathfrak{g}\left(A^{\prime}\right)$ corresponding to a generalized Cartan-Borcherds matrix $A^{\prime}$ is similar to (1.1.1). One should replace the last line of (1.1.1) by

$$
\left(\operatorname{ad} e_{i}\right)^{1-a_{i j}} e_{j}=\left(\operatorname{ad} f_{i}\right)^{1-a_{i j}} f_{j}=0 \text { if } i \neq j \text { and } a_{i i}=2 \text {, }
$$

and add the relation

$$
\left[e_{i}, e_{j}\right]=\left[f_{i}, f_{j}\right]=0 \text { if } a_{i j}=0 .
$$

Borcherds showed that generalized Kac-Moody algebras have similar properties to ordinary Kac-Moody algebras. They also have a denominator identity which has more general form than (1.1.3) and includes (1.3.7) as a particular case.

The identity (1.3.7) is the denominator identity for the generalized Kac-Moody algebra $\mathfrak{g}\left(A^{\prime}\right)$ where $A^{\prime}$ is the generalized Cartan-Borcherds matrix equals to the Gram matrix $A^{\prime}=\left(\left(\alpha, \alpha^{\prime}\right)\right), \alpha, \alpha^{\prime} \in P^{\prime}$ where

$$
P^{\prime}=P \cup 24 \rho \cup 24(2 \rho) \cup \cdots \cup 24(n \rho) \cup \cdots
$$

is the sequence of elements of the lattice $S$. Here $24(n \rho)$ means that we take the element $n \rho$ twenty four times to get the Gram matrix $A^{\prime}$. See details in [3], [4].

In (1.3.11), the set $P^{\prime}$ defining $A^{\prime}$ is called the set of simple roots. It is divided in the set $P^{\prime r e}=P$, described in (1.3.1), of simple real roots (they are orthogonal to the fundamental chamber $\mathcal{M}$ of the Weyl group $W$ and have positive square) and is the same as for the ordinary Kac-Moody algebra $\mathfrak{g}(A)$ defined by the generalized Cartan matrix $A$ in (1.3.3). The additional sequence

$$
P^{\prime \text { im }}=24 \rho \cup 24(2 \rho) \cup \cdots \cup 24(n \rho) \cup \cdots
$$

of $P^{\prime}$ (elements of $P^{\prime i m}$ have zero square) is defined by the Fourier coefficients in the sum part of the identity (1.3.7). For example, 24 in (1.3.12) is defined by the 24 in (1.3.5). Together $P^{\prime r e}$ and $P^{\prime \text { im }}$ define the generalized Cartan-Borcherds matrix $A^{\prime}$ and the generalized Kac-Moody algebra $\mathfrak{g}\left(A^{\prime}\right)$.

Borcherds example is very fundamental and beautiful. It has important applications in Mathematics, e. g. for moduli spaces of K3 and Enriques surface (see [8], [13] and [54]), and in Physics (e. g. in String Theory): it gives the Lie algebra of physical states of the Vertex Algebra of dimension 26 defined by the hyperbolic lattice $S$. See [2], [6], [11].

Many other similar examples of generalized Kac-Moody algebras and superalgebras graded by hyperbolic lattices and related with automorphic forms were found by Borcherds in [3] — [9], [12]. One of them when Lie algebras are graded by the unimodular even hyperbolic plane $H$ is very important for Borcherds proof of Moonshine Conjecture about modular properties of representations of the Monster 
1.4. A Theory of Lorentzian Kac-Moody algebras. Analyzing Borcherds example, one can suggest a general class of Lorentzian Kac-Moody algebras (or automorphic hyperbolic Kac-Moody algebras), see [36], [40], [41], [62], [64], [67]. They are defined by data (1) - (5) below:

(1) A hyperbolic lattice $S$ (i. e. a non-degenerate integral symmetric bilinear form of signature $(n, 1))$. It is the root lattice for the Lie algebra we want to construct. We follow [57] in notations and terminology related with lattices.

(2) A reflection group $W \subset O(S)$. It is generated by reflections in some set of roots of $S$. We remind that $\alpha \in S$ is called root if $\alpha^{2}>0$ and $\alpha^{2} \mid 2(\alpha, S)$. Any root defines a reflection $s_{\alpha}: x \mapsto x-\left(2(x, \alpha) / \alpha^{2}\right) \alpha, x \in S$, which gives an automorphism of the lattice $S$. The group $W$ is the Weyl group for the Lie algebra. We assume that the Weyl group $W$ is non-trivial. (For trivial $W$ the definition has to be changed, see [40].)

(3) A set $P=P(\mathcal{M})$ of orthogonal roots to the fundamental chamber $\mathcal{M} \subset$ $\mathcal{L}(S)=V^{+}(S) / \mathbb{R}_{++}$of $W$ and its subdivision $P=P_{\overline{0}} \cup P_{\overline{1}}$ by subsets of even and odd roots respectively. The set $P$ of roots of $S$ should have the property

$$
\mathcal{M}=\left\{\mathbb{R}_{++} x \in \mathcal{L}(S) \mid(x, P) \leq 0\right\}
$$

(the same as (1.3.2)) and should be minimal having this property (i. e. each face of $\mathcal{M}$ of highest dimension is orthogonal to exactly one element from $P$, and each element of $P$ is orthogonal to a face of $\mathcal{M}$ of highest dimension). We additionally require that $2 \alpha$ is a root of $S$ if $\alpha \in P_{\overline{1}}$ (thus $\alpha^{2} \mid(\alpha, S)$ ). Moreover, the set $P$ should have a Weyl vector $\rho \in S \otimes \mathbb{Q}$ which means that it satisfies the condition

$$
(\rho, \alpha)=-\alpha^{2} / 2, \quad \forall \alpha \in P
$$

(it is the same as (1.3.4)). ${ }^{2}$ The sets $P, P_{\overline{0}}$ and $P_{\overline{1}}$ are the sets of simple real roots, even simple real roots and odd simple real roots respectively for the Lie algebra. The set $P$ is not empty since $W$ is non-trivial. From (1.4.2), the Weyl vector $\rho$ is not zero too.

The main invariant of the data (1)-(3) is the generalized Cartan matrix

$$
A=\left(\frac{2\left(\alpha, \alpha^{\prime}\right)}{(\alpha, \alpha)}\right), \quad \alpha, \alpha^{\prime} \in P
$$

It defines data (1) - (3) up to some very clear equivalence.

(4) A holomorphic automorphic form $\Phi(z)$ of some weight $k,(k \in \mathbb{Z} / 2)$ on a IV type Hermitian symmetric domain, $z \in \Omega\left(V^{+}(S)\right)=\Omega(T)$, with respect to a subgroup $G \subset O^{+}(T)$ of finite index (the symmetry group of the Lie algebra) of an extended lattice $T=H(m) \oplus S$ (the symmetry lattice of the Lie algebra) where

\footnotetext{
${ }^{2}$ Existence of the Weyl vector $\rho$ from $S \otimes \mathbb{Q}$ is a very strong condition on the data (1)-(3). It would be better to call it as a lattice Weyl vector. We don't do it to simplify terminology. It is possible, that there exists a more general theory of Lorentzian Kac-Moody algebras when a Lie algebra can be graded by a degenerate hyperbolic lattice. Then the Weyl vector should be a linear function on the degenerate hyperbolic lattice, satisfying (1.4.2), and it always exists for an appropriate grading. It is easy to see that one should add a one-dimensional kernel only. On the other hand, if this theory exists, it seems, it is so general that the classification problem has no
} 
$H(m)=\left(\begin{array}{cc}0 & -m \\ -m & 0\end{array}\right), m \in \mathbb{N}$. (See [40] for more general definition.) Definition of the automorphic form $\Phi$ is the same as for the Borcherds example in Sect. 1.3. The only changes are: we identify $\Omega\left(V^{+}(S)\right)=\Omega(T)$ by the formula

$$
z \mapsto \mathbb{C} \omega_{z}, \quad z \in \Omega\left(V^{+}(S)\right), \omega_{z} \in \widetilde{\Omega}(T)
$$

where $\omega_{z}=(z, z) e_{1} / 2+e_{2} / m \oplus z$ and $e_{1}, e_{2}$ is the basis of the lattice $H(m)$ with the matrix above, and we assume that $\widetilde{\Phi}\left(\lambda \omega_{z}\right)=\lambda^{-k} \Phi(z), \lambda \in \mathbb{C}^{*}$, on the cone $\widetilde{\Omega}(T)$ has the property: $\widetilde{\Phi}(g \omega)=\chi(g) \widetilde{\Phi}(\omega)$ for any $g \in G$ where $\chi: G \rightarrow \mathbb{C}^{*}$ is a character or a multiplier system with the kernel of finite index in $G$.

The automorphic form $\Phi$ should have Fourier expansion of the form of denominator identity for a generalized Kac-Moody algebra with hyperbolic generalized Cartan-Borcherds matrix and which is agree with previous data (1) - (3). This form is

$$
\Phi(z)=\sum_{w \in W} \varepsilon(w)\left(\exp (-2 \pi i(w(\rho), z))-\sum_{a \in S \cap \mathbb{R}_{++} \mathcal{M}} m(a) \exp (-2 \pi i(w(\rho+a), z))\right)
$$

where $\varepsilon: W \rightarrow\{ \pm 1\}$ is a quadratic character (i. e. a homomorphism) such that $\varepsilon\left(s_{\alpha}\right)=(-1)^{1+\bar{i}}$ if $\alpha \in P_{\bar{i}}$ and $\bar{i}=\overline{0}, \overline{1}$. All Fourier coefficients $m(a)$ should be integral.

Let

$$
H=\left\{g \in O^{+}(S) \mid \Phi(g(z))= \pm \Phi(z)\right\}
$$

Since $\Phi$ is an automorphic form, this subgroup has finite index in $O^{+}(S)$. We have $W \subset H$. Let

$$
\operatorname{Sym}(\mathcal{M})=\left\{g \in O^{+}(S) \mid g(\mathcal{M})=\mathcal{M}\right\}
$$

be the symmetry group of the fundamental chamber $\mathcal{M}$ and

$$
\operatorname{Sym}\left(P_{\overline{1}} \subset P\right)=\left\{g \in O^{+}(S) \mid g(P)=P \& g\left(P_{\overline{1}}\right)=P_{\overline{1}}\right\}
$$

its subgroup which keeps invariant the set $P$ of orthogonal roots to $\mathcal{M}$, and its subset $P_{\overline{1}}$ as well.

(*) We assume that there exists a subgroup $A \subset \operatorname{Sym}\left(P_{\overline{1}} \subset P\right)$ such that $A \subset H$ and the semi-direct product $W \rtimes A$ has finite index in $H$. It follows that $A$ is a subgroup of finite index in $\operatorname{Sym}\left(P_{\overline{1}} \subset P\right)$ and in $\operatorname{Sym}(\mathcal{M})$, and $W \rtimes A$ has finite index in $O^{+}(S)$. In particular, subgroups $W \rtimes S y m\left(P_{\overline{1}} \subset P\right)$ and $W \rtimes S y m(\mathcal{M})$ have finite index in $O(S)$. The automorphic form $\Phi$ defines the set of simple imaginary roots and gives the denominator identity of the Lie algebra. Using automorphic properties of $\Phi(z)$, it is good to calculate the infinite product part of the denominator identity

$$
\Phi(z)=\exp (-2 \pi i(\rho, z)) \prod_{\alpha \in \Delta_{+}}(1-\exp (-2 \pi i(\alpha, z)))^{\operatorname{mult}(\alpha)}
$$

which gives multiplicities mult $(\alpha)$ of roots $\alpha$ of the Lie algebra. See below. There 
Like for Borcherds example, already data (1) - (4) define a generalized KacMoody algebra or superalgebra. See the definition below. But it was understood that it is good to suppose (at least, to have finiteness results) the additional condition:

(5) The automorphic form $\Phi$ on the domain $\Omega\left(V^{+}(S)\right)=\Omega(T)$ should be reflective. It means that the divisor of $\Phi$ is union of rational quadratic divisors which are orthogonal to some roots of the extended lattice $T$. Here for a root $\alpha \in T$ (the definition of a root of $T$ is the same as for the lattice $S$ ) the quadratic divisor orthogonal to $\alpha$ is equal to

$$
D_{\alpha}=\{\mathbb{C} \omega \in \Omega(T) \mid(\omega, \alpha)=0\}
$$

The property (5) is valid for Borcherds example above and for the most part of known cases. Moreover, it is true in the neighbourhood of the cusp where the infinite product (1.4.9) converges. Thus, we want it to be true globally.

Below we give the definition of a generalized Kac-Moody superalgebra $\mathfrak{g}$ corresponding to data (1)-(4). It is given by the sequence $P^{\prime} \rightarrow S$ of simple roots. This sequence is divided in a sequence $P^{\prime \text { re }}$ of simple real roots and a sequence $P^{\prime \text { im }}$ of simple imaginary roots. Both these sequences are also divided in the sequences of even and odd roots marked by $\overline{0}$ and $\overline{1}$ respectively. We set $P^{\prime \text { re }}=P, P^{\prime \text { re }}=P_{\bar{i}}$ where $P, P_{\bar{i}}, \bar{i}=\overline{0}, \overline{1}$, are defined in the datum (3).

For any primitive $0 \neq a \in S \cap \mathbb{R}_{++} \mathcal{M}$ with $(a, a)=0$, one should find $\tau(n a) \in \mathbb{Z}$, $n \in \mathbb{N}$, from the identity with a formal variable $t$ :

$$
1-\sum_{k \in \mathbb{N}} m(k a) t^{k}=\prod_{n \in \mathbb{N}}\left(1-t^{n}\right)^{\tau(n a)} .
$$

We set

$$
\begin{aligned}
& P^{\prime} \overline{0}=\left\{m(a) a \mid a \in S \cap \mathbb{R}_{++} \mathcal{M},(a, a)<0 \text { and } m(a)>0\right\} \cup \\
& \cup\left\{\tau(a) a \mid a \in S \cap \mathbb{R}_{++} \mathcal{M},(a, a)=0 \text { and } \tau(a)>0\right\} ; \\
& P^{\prime \frac{i m}{1}}=\left\{-m(a) a \mid a \in S \cap \mathbb{R}_{++} \mathcal{M},(a, a)<0 \text { and } m(a)<0\right\} \cup \\
& \cup\left\{-\tau(a) a \mid a \in S \cap \mathbb{R}_{++} \mathcal{M},(a, a)=0 \text { and } \tau(a)<0\right\} .
\end{aligned}
$$

Here $k a$ means that we repeat the element $a$ exactly $k$ times for the sequence. The generalized Kac-Moody superalgebra $\mathfrak{g}$ is a Lie superalgebra generated by $h_{r}, e_{r}$, $f_{r}$ where $r \in P^{\prime}$. All generators $h_{r}$ are even, generators $e_{r}, f_{r}$ are even (respectively odd) if $r$ is even (respectively odd). They have the defining relations 1) - 5) below:

1) The map $r \mapsto h_{r}$ for $r \in P^{\prime}$ gives an embedding of $S \otimes \mathbb{C}$ to $\mathfrak{g}$ as an Abelian subalgebra (it is even);

2) $\left[h_{r}, e_{r^{\prime}}\right]=\left(r, r^{\prime}\right) e_{r^{\prime}}$ and $\left[h_{r}, f_{r^{\prime}}\right]=-\left(r, r^{\prime}\right) f_{r^{\prime}}$;

3) $\left[e_{r}, f_{r^{\prime}}\right]=h_{r}$ if $r=r^{\prime}$, and is 0 if $r \neq r^{\prime}$;

4) $\left(a d e_{r}\right)^{1-2\left(r, r^{\prime}\right) /(r, r)} e_{r^{\prime}}=\left(a d f_{r}\right)^{1-2\left(r, r^{\prime}\right) /(r, r)} f_{r^{\prime}}=0$ if $r \neq r^{\prime}$ and $(r, r)>0$ (equivalently, $r \in P^{\prime r e}$ );

5) If $\left(r, r^{\prime}\right)=0$, then $\left[e_{r}, e_{r^{\prime}}\right]=\left[f_{r}, f_{r^{\prime}}\right]=0$.

See [3], [5], [36], [40], [69] for details. We remark that for Lie algebras this definition is equivalent to the definition above using the generalized Cartan-Borcherds 
The algebra $\mathfrak{g}$ is graded by the root lattice $S$ where the generators $h_{r}, e_{r}$ and $f_{r}$ have the weights $0, r \in S$ and $-r \in S$ respectively. We have

$$
\mathfrak{g}=\bigoplus_{\alpha \in S} \mathfrak{g}_{\alpha}=\mathfrak{g}_{0} \bigoplus\left(\bigoplus_{\alpha \in \Delta_{+}} \mathfrak{g}_{\alpha}\right) \bigoplus\left(\bigoplus_{\alpha \in \Delta_{+}} \mathfrak{g}_{-\alpha}\right)
$$

where $\mathfrak{g}_{0}=S \otimes \mathbb{C}$, and $\Delta$ is the set of roots (i. e. $\alpha \in S$ with $\mathfrak{g}_{\alpha} \neq 0$ ). A root $\alpha$ is called positive $\left(\alpha \in \Delta_{+}\right)$if $(\alpha, \mathcal{M}) \leq 0$.

For a root $\alpha \in \Delta$ the multiplicity of $\alpha$ is equal to $\operatorname{mult}(\alpha)=\operatorname{dim} \mathfrak{g}_{\alpha, \overline{0}}-\operatorname{dim} \mathfrak{g}_{\alpha, \overline{1}}$. Multiplicities mult $(\alpha)$ of roots and the numbers $m(a)$ defining generators of $\mathfrak{g}$ are related by the denominator identity (due to Weyl, Kac, Borcherds)

$$
\begin{aligned}
& \exp (-2 \pi i(\rho, z)) \prod_{\alpha \in \Delta_{+}}(1-\exp (-2 \pi i(\alpha, z)))^{\operatorname{mult}(\alpha)} \\
& =\sum_{w \in W} \varepsilon(w)\left(\exp (-2 \pi i(w(\rho), z))-\sum_{a \in S \cap \mathbb{R}_{++} \mathcal{M}} m(a) \exp (-2 \pi i(w(\rho+a), z))\right),
\end{aligned}
$$

which identifies multiplicities of factors in (1.4.9) and multiplicities of roots of the algebra $\mathfrak{g}$. See [3], [5], [47], [48], [36], [69].

The generalized Kac-Moody superalgebras $\mathfrak{g}$ above given by the data (1)-(5) constitute the Theory of Lorentzian Kac-Moody algebras (or automorphic Lorentzian Kac-Moody algebras) which we consider.

By (4), they have similar property to the property (II) from Sect. 1.2 for finite and affine algebras: their denominator identities give automorphic forms. For Lorentzian case, they are automorphic forms on IV type Hermitian symmetric domains.

What is about a similar property to the property (I) from Sect. 1.2 for finite and affine Kac-Moody algebras? How many data (1) - (5) one may have? This is the main subject of the paper. We consider that in the next sections.

1.5. Finiteness results about hyperbolic root systems (data (1) - (3)) of Lorentzian Kac-Moody algebras. To classify finite and affine Kac-Moody algebras, one need to classify appropriate finite and affine root systems (authors don't know any other method). They are classified, and their classification gives classification of finite and affine Lie algebras.

Data (1) - (5) define Lorentzian Kac-Moody algebras. One can consider the set of possible data (1) - (3) from data (1) - (5) as hyperbolic root systems which are appropriate for Lorentzian Kac-Moody algebras. Main result is that they satisfy very restricted conditions, and it is possible to classify them, in principle; this makes the theory of Lorentzian Kac-Moody algebras similar to theories of finite and affine Kac-Moody algebras. Moreover, their number is in essential finite when rk $S \geq 3$. It means that Lorentzian Kac-Moody algebras for $\mathrm{rk} S \geq 3$ are exceptional, like exceptional simple Lie algebras $\mathbb{E}_{6}, \mathbb{E}_{7}, \mathbb{E}_{8}, \mathbb{F}_{4}, \mathbb{G}_{2}$. More generally, for these results, we can drop the condition (5) considering the set of possible data (1) - (3) in data (1) - (4).

Since we assume that Weyl group is non-trivial, $\mathrm{rk} S \geq 2$. If $\mathrm{rk} S=2$, then $P$ has one or two elements, and classification of data (1) - (3) in data (1) - (4) is 
Further we assume that rk $S \geq 3$.

From the condition $(*)$ in $(4)$, we have that

$$
W \rtimes \operatorname{Sym}(\mathcal{M}) \text { has finite index in } O^{+}(S) \text {. }
$$

Non-trivial reflection subgroups $W \subset O(S)$ having this property are called of restricted arithmetic type (we follow the terminology in [64, Sect. 1.4]). It follows [64, Theorem 1.4.3] that $W$ has arithmetic type which means that

$$
\{x \in S \otimes \mathbb{R} \mid(x, P) \leq 0\} \subset \mathbb{R}_{++} \mathcal{M} \subset \overline{V^{+}(S)} .
$$

In particular, the Weyl vector $\rho \in \mathbb{R}_{++} \mathcal{M} \subset \overline{V^{+}(S)}$ and $\rho^{2} \leq 0$. By (1.4.2), the $\rho$ is non-zero.

From (1.5.2) one can deduce that the set $P$ generates $S \otimes \mathbb{Q}$. From (1.4.2), it is easy to see that the Weyl vector $\rho$ is unique and it is invariant with respect to $\operatorname{Sym}\left(P_{\overline{1}} \subset P\right)$ which has finite index in $\operatorname{Sym}(\mathcal{M})$. One can get the same using Fourier expansion (1.4.5). Really, $\exp (-2 \pi i(\rho, z))$ is one of Fourier harmonics of the holomorphic automorphic form $\Phi(z)$. It follows that $\rho^{2} \leq 0$. Otherwise, the automorphic form $\Phi(z)$ has poles. The subgroup $H \subset O^{+}(S)$ of finite index, in (1.4.6), preserves $\pm \Phi(z)$ and the set of Fourier harmonics $\varepsilon(w) m(a) \exp (-2 \pi i(w(\rho+a), z))$, $a \in S \cap \mathbb{R}_{++} \mathcal{M}, m(a) \neq 0, w \in W$. For Fourier harmonics $c(x) \exp (-2 \pi i(x, z))$ and $c(y) \exp (-2 \pi i(y, z))$, we say that $x \geq y$, if $x-y \in \overline{V^{+}(S)}$. The subgroup $H$ preserves this ordering and permutes its minimal elements $\varepsilon(w) \exp (-2 \pi i(w(\rho), z))$, $w \in W$. Let $H_{\rho} \subset H$ be the stabilizer subgroup of $\rho$. Then $W \rtimes H_{\rho}=H$ has finite index in $O^{+}(S)$. From definition of $\rho$, it follows that $H_{\rho} \subset \operatorname{Sym}(\mathcal{M})$. It follows that $\rho$ is invariant for the subgroup $H_{\rho} \subset \operatorname{Sym}(\mathcal{M})$ of finite index. Remark that we did not use the additional condition $(*)$ in (4) under this consideration.

Thus, the orbit

$$
\operatorname{Sym}(\mathcal{M})(\rho) \text { is finite. }
$$

Here $\rho \in S \otimes \mathbb{Q}$ and $\rho \neq 0$. For some $m \in \mathbb{N}$, the element $r=m \rho \in S, r \neq 0$ and $\operatorname{Sym}(\mathcal{M})(r)$ is also finite.

Hyperbolic lattices $S$ having the properties similar to (1.5.1) and (1.5.3) for some of their reflection subgroup $W$ are called reflective. Here is the exact definition.

Definition 1.5.1. A hyperbolic lattice $M$ is called reflective if there exists a reflection subgroup $W \subset O(M)$ with a fundamental chamber $\mathcal{M} \subset V^{+}(M) / \mathbb{R}_{++}$ and the symmetry group $\operatorname{Sym}(\mathcal{M})$ of the fundamental chamber $\mathcal{M}$ such that the corresponding semi-direct product $W \rtimes S y m(\mathcal{M})$ has finite index in $O(M)$ (i. e. $W$ has restricted arithmetic type), and there exists a non-zero $r \in M$ such that the orbit $\operatorname{Sym}(\mathcal{M})(r)$ is finite. The element $r$ is called a generalized Weyl vector for the reflection group $W$ and its fundamental chamber $\mathcal{M}$.

Thus, a hyperbolic lattice $M$ is reflective if it has a reflection group $W \subset O(M)$ having restricted arithmetic type and a generalized Weyl vector for its fundamental chamber. The group $W$ has elliptic, parabolic and hyperbolic type, if it has a generalized Weyl vector $r$ respectively with $r^{2}<0$, with $r^{2}=0$ and no generalized Weyl vectors with $r^{2}<0$, and with $r^{2}>0$ and no generalized Weyl vectors with $r^{2} \leq 0$.

It is easy to see that a hyperbolic lattice $M$ is reflective if and only if its full 
fundamental chamber have a generalized Weyl vector. The group $W(M)$ obviously has restricted arithmetic type since it is normal in $O(M)$.

Thus, we have

Proposition 1.5.2. Assume that $r k S \geq 3$. Then any data (1) - (3) from data (1) - (4) satisfy the conditions: the Weyl vector $\rho \in S \otimes \mathbb{Q}$ is not zero and $\rho^{2} \leq 0$;

$W \rtimes \operatorname{Sym}(\mathcal{M})$ has finite index in $O(S)$, and $\operatorname{Sym}(\mathcal{M})(\rho)$ is finite.

In particular, the hyperbolic root lattice $S$ is reflective, the Weyl group $W$ has restricted arithmetic type, the Weyl vector $\rho$ is a generalized Weyl vector for $W$ and $\mathcal{M} ;^{3}$

$$
\operatorname{Sym}\left(P_{\overline{1}} \subset P\right) \text { has finite index in } \operatorname{Sym}(\mathcal{M}) \text {, }
$$

and

$$
\operatorname{Sym}\left(P_{\overline{1}} \subset P\right)(\rho)=\rho .
$$

We have the following general crucial result which, in particular, gives finiteness for the set of root lattices in (1).

Theorem 1.5.3. The set or reflective hyperbolic lattices $M$ of $r k M \geq 3$ is finite (up to isomorphism) if we consider lattices up to multiplication of their forms by positive rational numbers.

In particular, the set of hyperbolic root lattices $S$ of rk $S \geq 3$ for data (1) in data (1) - (4) is finite if we consider lattices up to multiplication of their forms by positive rational numbers.

If rank $\geq 3$ is fixed, see the proof for elliptic case in [59], [60]; for parabolic case in [64], [68]; for hyperbolic case in [66], [68]. Boundendess of the rank follows from results in [60], [63], [71].

For fixed rank the proof of Theorem 1.5.3 follows from

Lemma 1.5.4 (about a narrow part of the polyhedron $\mathcal{M}$ ). Let $M$ be a reflective hyperbolic lattice of rank $n=r k M \geq 3$ with a reflection group $W$ of restricted arithmetic type and with a fundamental chamber $\mathcal{M}$ having a generalized Weyl vector. Let $P(\mathcal{M})$ be the set of orthogonal vectors to $\mathcal{M}$ directed outwards of $\mathcal{M}$.

Then there exist $\alpha_{1}, \alpha_{2}, \ldots, \alpha_{n}$ from $P(\mathcal{M})$ with the properties:

(a) $\alpha_{1}, \alpha_{2}, \ldots, \alpha_{n}$ generate $M \otimes \mathbb{Q}$;

(b) $\frac{4\left(\alpha_{i}, \alpha_{j}\right)^{2}}{\alpha_{i}^{2} \alpha_{j}^{2}}<100^{2}$ for any $1 \leq i, j \leq n$;

(c) the Gram graph of $\alpha_{1}, \alpha_{2}, \ldots, \alpha_{n}$ is connected, $i$. e. one cannot divide the set $\alpha_{1}, \alpha_{2}, \ldots, \alpha_{n}$ in two orthogonal to each other non-empty subsets.

We remark that for roots $\alpha_{1}, \alpha_{2}, \ldots, \alpha_{n}$ the numbers $\frac{4\left(\alpha_{i}, \alpha_{j}\right)^{2}}{\alpha_{i}^{2} \alpha_{j}^{2}}$ are integral. The proof of Lemma 1.5.4 is based on study of geometry of the convex polyhedra $\mathcal{M}$. Actually, Lemma 1.5.4 is valid for all convex polyhedra having properties similar to $\mathcal{M}$. The main property is that the polyhedron $\mathcal{M}$ is convex locally finite and

\footnotetext{
${ }^{3}$ Remark that all the statements above are valid without the additional condition $(*)$ in (4), and, if necessary, one can drop this additional condition, considering more general class
} 
has almost finite volume. Here, almost finite volume means the following. For elliptic case $\mathcal{M}$ is a finite polyhedron of finite volume. For parabolic case, $\mathcal{M}$ is finite and has finite volume in any angle with the vertex $\mathbb{R}_{++} r$ and over a compact set on the horosphere (one has the same property for the Borcherds example from Sect. 1.3). For hyperbolic case, $\mathcal{M}$ is finite and has finite volume in any orthogonal cylinder over compact set in the hyperplane which is orthogonal to $r$. Here $r$ is a generalized Weyl vector for $\mathcal{M}$. For example, Lemma 1.5.4 is valid for any finite convex polyhedron of finite volume in a hyperbolic space of dimension $n-1 \geq 2$. For parabolic and hyperbolic cases, one has to add also some conditions of periodicity of $\mathcal{M}$. The proof of Lemma 1.5.4 is not trivial, and it is the most hard for the hyperbolic case.

Theorem 1.5.3 requires existence of a generalized Weyl vector $r$ only. If there exists a Weyl vector $\rho$ (satisfying (1.4.2)), we can prove finiteness results also for data (1) - (3) from (1) — (4). Two data (1) - (3) are called isomorphic if they can be identified by an isomorphism of hyperbolic lattices in (1). We also accept multiplication of the forms of lattices $S$ in (1) by positive rational numbers. We know that data (1) — (3) from data (1) — (4) satisfy conditions (1.5.4) — (1.5.6) of Proposition 1.5.2. One can consider data (1) - (3) satisfying additional conditions (1.5.4) - (1.5.6) as hyperbolic root systems which are appropriate for Lorentzian Kac-Moody algebras theory. From Theorem 1.5.3 and Lemma 1.5.4, one can deduce

Theorem 1.5.5. Assume that $r k S \geq 3$.

Then the set of data (1) - (3) satisfying conditions (1.5.4) - (1.5.6) is empty if $\rho^{2}>0$ (i.e. for hyperbolic case); finite if $\rho^{2}<0$ (i.e. for elliptic case). It is also finite if $\rho^{2}=0$ (i.e. for parabolic case), if one fixes a constant $C>0$ and additionally requires $\left[O^{+}(S)_{\rho}: \operatorname{Sym}\left(P_{\overline{1}} \subset P\right)\right]<C$ where $O^{+}(S)_{\rho}$ is the stabilizer subgroup of $\rho$ in $\mathrm{O}^{+}(S)$.

See the proof in [64, Theorem 1.3.3]. We mention that for $\rho^{2}=0$ the group $\mathrm{O}^{+}(S)_{\rho}$ is a (rk $\left.S-2\right)$-dimensional crystallographic group acting in Euclidean affine space related with the semi-positive lattice $(\rho)_{S}^{\perp}$. In particular, it contains $\mathbb{Z}^{\text {rk } S-2}$ as a subgroup of translations of finite index. If the constant $C \rightarrow+\infty$, number of cases for the parabolic case tends to infinity, see [64, Example 1.3.4]. Existence of Weyl vector $\rho$ means geometrically that faces of $\mathcal{M}$ of highest dimension orthogonal to $\alpha \in P$ are touching a sphere with the center $\mathbb{R}_{++} \rho$ and radius depending on $\alpha^{2}$ where $\alpha^{2}$ is bounded by a constant depending on $S$. It makes polyhedra $\mathcal{M}$ very special, and implies finiteness of their set.

As an example, let us prove Theorem 1.5.5 for elliptic case $\left(\rho^{2}<0\right)$ and fixed $n=\operatorname{rk} S \geq 3$.

By Theorem 1.5.3, the set of root lattices $S$ is finite. We fix one of them.

Let $\alpha$ be a root of $S$. Let $\alpha=m \alpha_{0}$ where $\alpha_{0}$ is a primitive $\operatorname{root}$ of $S$ and $m \in \mathbb{N}$. Since $\alpha$ is a root, $\left(\frac{2 \alpha}{\alpha^{2}}, S\right) \in \mathbb{Z}$. Thus, $\frac{\alpha_{0}}{m \alpha_{0}^{2}} \in 2^{-1} S^{*}$ where $S^{*}=\operatorname{Hom}(S, \mathbb{Z})$ is the dual lattice. It follows that $m \alpha_{0}^{2} \mid 2 \lambda$ where $\lambda$ is the exponent (i.e. the maximal order of elements) of the finite Abelian group $S^{*} / S$. Thus, $m, \alpha_{0}^{2}$ and $\alpha^{2}=m^{2} \alpha_{0}^{2}$ are bounded by a constant depending on $S$.

By Lemma 1.5.4, there are roots $\alpha_{1}, \ldots, \alpha_{n}$ from $P$ which satisfy conditions (a) and (b) of the lemma. Numbers $\frac{4\left(\alpha_{i}, \alpha_{j}\right)^{2}}{\alpha_{i}^{2} \alpha_{j}^{2}}$ are integral and are less then $100^{2}$. Since $\alpha_{i}^{2}$ are bounded, it follows that the set of possible Gram matrices $\left(\left(\alpha_{i}, \alpha_{j}\right)\right)$, 
Since $\alpha_{1}, \ldots, \alpha_{n}$ generate $S \otimes \mathbb{Q}$ and the lattice $S$ is non-degenerate, there exists a unique $\rho \in S \otimes \mathbb{Q}$ such that $\left(\rho, \alpha_{i}\right)=-\alpha_{i}^{2} / 2, i=1, \ldots, n$. It is the Weyl vector $\rho$ for $P$.

All elements $\alpha \in P$ satisfy the condition $(\rho, \alpha)=-\alpha^{2} / 2$ where $0<\alpha^{2}<K$ for some constant $K$ depending on $S$. Thus, $P$ is a subset of the set of elements $a \in S$ satisfying conditions $-K / 2<(\rho, a)<0$ and $0<a^{2}<K$. If $\rho^{2}<0$ the set is finite because the lattice $S$ is hyperbolic (has exactly one negative square). It follows, that $P$ is finite, and there exists only a finite number of possibilities for $P$ and its subset $P_{\overline{1}}$. It follows Theorem 1.5.5 for elliptic case.

The parabolic case (when $\rho^{2}=0$ and $\rho \neq 0$ ) requires additional simple considerations with the crystallographic group $O(S)_{\rho}$ and its action on the set $P$ which is infinite for this case. The group $\operatorname{Sym}\left(P_{\overline{1}} \subset P\right)$ has finite index in $O(S)_{\rho}$ and has finite number of orbits in $P$.

In this proof, formally we did not use the condition (c) of Lemma 1.5.4, but it is very important for the proof of Theorem 1.5.3 which also has been used. As we see, for the proof not only Theorem 1.5.3 is important, but also the method of its proof (Lemma 1.5.4) is very important.

Let us give an example of classification of data (1) - (3) satisfying conditions $(1.5 .4)-(1.5 .6)$.

Theorem 1.5.6. Let us consider all data (1) -(3) satisfying conditions (1.5.4) (1.5.6) and such that additionally $r k S=3$, all roots $\alpha \in P$ have equal squares $\alpha^{2}$ (equivalently, the generalized Cartan matrix (1.4.3) of $P$ is symmetric), and the Weyl vector $\rho$ has $\rho^{2}<0$ (elliptic type).

Then the generalized Cartan matrix of $P$ is one of the following 16 symmetric matrices $A_{i, j}$ and $B_{j}$ below:

$$
\begin{aligned}
& A_{1,0}=\left(\begin{array}{rrr}
2 & 0 & -1 \\
0 & 2 & -2 \\
-1 & -2 & 2
\end{array}\right), \quad A_{1, I}=\left(\begin{array}{rrr}
2 & -2 & -1 \\
-2 & 2 & -1 \\
-1 & -1 & 2
\end{array}\right), \quad A_{1, I I}=\left(\begin{array}{rrr}
2 & -2 & -2 \\
-2 & 2 & -2 \\
-2 & -2 & 2
\end{array}\right) \\
& A_{1, I I I}=\left(\begin{array}{rrrrc}
2 & -2 & -6 & -6 & -2 \\
-2 & 2 & 0 & -6 & -7 \\
-6 & 0 & 2 & -2 & -6 \\
-6 & -6 & -2 & 2 & 0 \\
-2 & -7 & -6 & 0 & 2
\end{array}\right) ; \quad A_{2,0}=\left(\begin{array}{rrr}
2 & -2 & -2 \\
-2 & 2 & 0 \\
-2 & 0 & 2
\end{array}\right), \quad A_{2, I}=\left(\begin{array}{rrrr}
2 & -2 & -4 & 0 \\
-2 & 2 & 0 & -4 \\
-4 & 0 & 2 & -2 \\
0 & -4 & -2 & 2
\end{array}\right), \\
& A_{2, I I}=\left(\begin{array}{rrrr}
2 & -2 & -6 & -2 \\
-2 & 2 & -2 & -6 \\
-6 & -2 & 2 & -2 \\
-2 & -6 & -2 & 2
\end{array}\right), \quad A_{2, I I I}=\left(\begin{array}{rrrrrrrr}
2 & -2 & -8 & -16 & -18 & -14 & -8 & 0 \\
-2 & 2 & 0 & -8 & -14 & -18 & -16 & -8 \\
-8 & 0 & 2 & -2 & -8 & -16 & -18 & -14 \\
-16 & -8 & -2 & 2 & 0 & -8 & -14 & -18 \\
-18 & -14 & -8 & 0 & 2 & -2 & -8 & -16 \\
-14 & -18 & -16 & -8 & -2 & 2 & 0 & -8 \\
-8 & -16 & -18 & -14 & -8 & 0 & 2 & -2 \\
0 & -8 & -14 & -18 & -16 & -8 & -2 & 2
\end{array}\right) ; \\
& A_{3,0}=\left(\begin{array}{rrr}
2 & -2 & -2 \\
-2 & 2 & -1 \\
-2 & -1 & 2
\end{array}\right), \quad A_{3, I}=\left(\begin{array}{rrrr}
2 & -2 & -5 & -1 \\
-2 & 2 & -1 & -5 \\
-5 & -1 & 2 & -2 \\
-1 & -5 & -2 & 2
\end{array}\right) \\
& A_{3, I I}=\left(\begin{array}{rrrrrr}
2 & -2 & -10 & -14 & -10 & -2 \\
-2 & 2 & -2 & -10 & -14 & -10 \\
-10 & -2 & 2 & -2 & -10 & -14 \\
-14 & -10 & -2 & 2 & -2 & -10 \\
-10 & -14 & -10 & -2 & 2 & -2
\end{array}\right)
\end{aligned}
$$




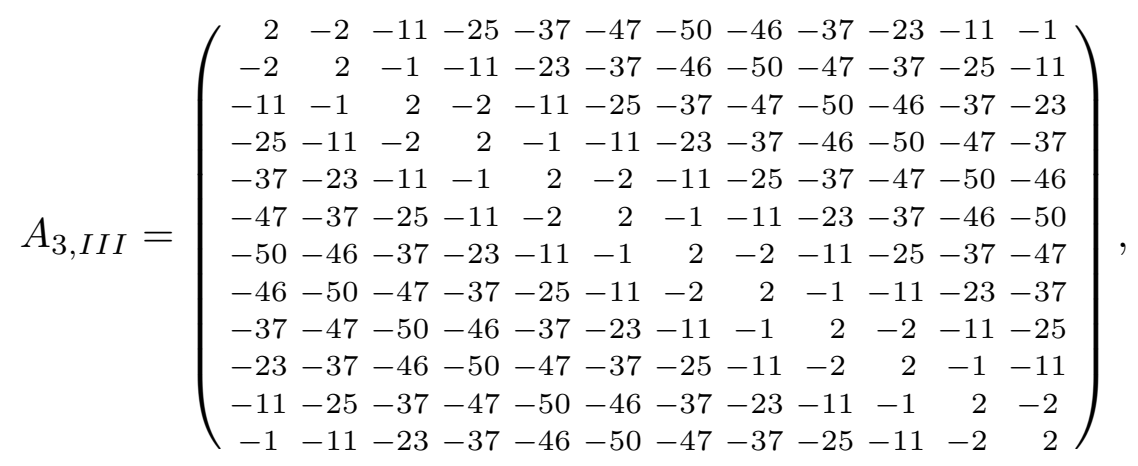

$$
\begin{aligned}
& B_{1}=\left(\begin{array}{rrrr}
2 & 0 & -3 & -1 \\
0 & 2 & -1 & -3 \\
-3 & -1 & 2 & 0 \\
-1 & -3 & 0 & 2
\end{array}\right), \quad B_{2}=\left(\begin{array}{rrrr}
2 & -1 & -4 & -1 \\
-1 & 2 & -1 & -4 \\
-4 & -1 & 2 & -1 \\
-1 & -4 & -1 & 2
\end{array}\right) \\
& B_{3}=\left(\begin{array}{rrrrrr}
2 & 0 & -4 & -6 & -4 & 0 \\
0 & 2 & 0 & -4 & -6 & -4 \\
-4 & 0 & 2 & 0 & -4 & -6 \\
-6 & -4 & 0 & 2 & 0 & -4 \\
-4 & -6 & -4 & 0 & 2 & 0 \\
0 & -4 & -6 & -4 & 0 & 2
\end{array}\right), \quad B_{4}=\left(\begin{array}{rrrrrr}
2 & -1 & -7 & -10 & -7 & -1 \\
-1 & 2 & -1 & -7 & -10 & -7 \\
-7 & -1 & 2 & -1 & -7 & -10 \\
-10 & -7 & -1 & 2 & -1 & -7 \\
-7 & -10 & -7 & -1 & 2 & -1 \\
-1 & -7 & -10 & -7 & -1 & 2
\end{array}\right) .
\end{aligned}
$$

For all these cases the fundamental chamber $\mathcal{M}$ is a closed polygon on the hyperbolic plane with angles respectively:

$A_{1,0}: \pi / 2,0, \pi / 3 ; A_{1, I}: 0, \pi / 3, \pi / 3 ; A_{1, I I}: 0,0,0 ; A_{1, I I I}: 0, \pi / 2,0, \pi / 2,0$ $A_{2,0}: 0, \pi / 2,0 ; A_{2, I}: 0, \pi / 2,0, \pi / 2 ; A_{2, I I}: 0,0,0,0$

$A_{2, I I I}: 0, \pi / 2,0, \pi / 2,0, \pi / 2,0, \pi / 2$

$A_{3,0}: 0, \pi / 3,0 ; A_{3, I}: 0, \pi / 3,0, \pi / 3 ; A_{3, I I}: 0,0,0,0,0,0$

$A_{3, I I I}: 0, \pi / 3,0, \pi / 3,0, \pi / 3,0, \pi / 3,0, \pi / 3,0, \pi / 3$.

$B_{1}: \pi / 2, \pi / 3, \pi / 2, \pi / 3 ; \quad B_{2}: \pi / 3, \pi / 3, \pi / 3, \pi / 3$

$B_{3}: \pi / 2, \pi / 2, \pi / 2, \pi / 2, \pi / 2, \pi / 2 ; B_{4}: \pi / 3, \pi / 3, \pi / 3, \pi / 3, \pi / 3, \pi / 3$.

All these polygons are touching a circle with the center $\mathbb{R}_{++} \rho$ where $\rho$ is the Weyl vector. The first 12 matrices $A_{i, j}$ give non-compact polygons (they have at least one zero angle). The last four matrices $B_{i}$ give compact polygons.

See the proof in [40, Theorems 1.2.1 and 1.3.1]. The proof is based on Lemma 1.5.4 and uses computer calculations. They follow the proof of Theorem 1.5.5 which we outlined above.

Theorem 1.5.6 gives classification of generalized Cartan matrices of the possible sets $P$, but one can get from here description of the corresponding data $(1)-(3)$ as follows. Let $A=\left(a_{i j}\right), 1 \leq i, j \leq m$, be one of generalized Cartan matrices of Theorem 1.5.6. Let us consider a free $\mathbb{Z}$-module $\widetilde{M}=\oplus_{i=1}^{m} \mathbb{Z} \widetilde{\alpha}_{i}$ with the symmetric bilinear form $\left(\left(\widetilde{\alpha}_{i}, \widetilde{\alpha}_{j}\right)\right)=A$. The matrix $A$ has rank three, and $\widetilde{M}$ modulo the kernel of this form defines a hyperbolic lattice $M$ of the rank three. It is generated by images $\alpha_{i}$ of $\tilde{\alpha}_{i}$. Any its integral overlattice $M \subset S$ of finite index can be taken for the datum (1). Their number is finite since $S \subset M^{*}$. The set $P$ is given by all elements $\alpha_{i}$. Since $\alpha_{i}^{2}=2$, all elements $\alpha_{i}$ are roots of $S$. Reflections in roots of $P$ generate the reflection group $W \subset O(S)$. Its fundamental chamber is equal to $\mathcal{M}=\{0 \neq x \in S \otimes \mathbb{R} \mid(x, P) \leq 0\} / \mathbb{R}_{++} \subset \overline{\mathcal{L}(S)}=\overline{V^{+}(S) / \mathbb{R}_{++}}$. As a subset $P_{\overline{1}} \subset P$ one can take any number of roots $\alpha_{i} \in P$ which satisfy the condition $\alpha_{i}^{2}=2 \mid\left(\alpha_{i}, S\right)$. One can check (it depends on the generalized Cartan matrix $A$ only) that all these data satisfy conditions (1) - (3) and conditions (1.5.4) - 
Almost for all matrices $A_{i, j}$ of Theorem 1.5.6 and corresponding data (1) - (3) one can construct additional data (4) satisfying (5). See [36], [37], [39], [41]. We give almost all these examples in Sect. 2.6 below.

Remark 1.5.7. Formally, for the considered theory of Lorentzian Kac-Moody algebras which are given by data (1)-(5), it is sufficient to consider only reflective hyperbolic lattices $S$ with a Weyl vector $\rho$. In particular, $\rho^{2} \leq 0$, if $\operatorname{rk} S \geq 3$, and all these lattices $S$ are reflective of elliptic or parabolic type.

There are several reasons why it is important and necessary to consider arbitrary reflective hyperbolic lattices with arbitrary generalized Weyl vector (having a square of any sign, in particular).

First, finiteness results are valid for general reflective hyperbolic lattices. For the classification of reflective hyperbolic lattices with a Weyl vector in general, it is necessary first to find maximal reflective hyperbolic lattices with a generalized Weyl vector, and then to find all their sublattices of finite index having a Weyl vector. In practice, it is impossible to consider reflective hyperbolic lattices with a Weyl vector and with a generalized Weyl vector separately.

Second, it seems, there is a more general class of Lie algebras (analogous to Lorentzian Kac-Moody algebras which we consider here) such that for this class it is necessary to consider reflective hyperbolic lattices and identities similar to (1.4.14) with a generalized Weyl vector $\rho$ having square with any sign. In $\S 2$ we shall give and classify many such identities. Very few of them are related with a Weyl vector $\rho$. It may be similar to McDonald identities which were first discovered and later found to be related with affine Kac-Moody algebras. Results of [26] and [50] give this hope.

From our point of view, all reflective hyperbolic lattices and identities analogous to (1.4.14) with a generalized Weyl vector $\rho$, should be interesting for one or another theory of Lie algebras which is analogous to the theory of Lorentzian Kac-Moody algebras which we consider here. It is a very interesting problem to understand their importance from Lie algebras point of view.

\subsection{Finiteness conjectures and results about data (4), (5) for Lorentzian} Kac-Moody algebras. Here we follow [40] and [65].

We expect that data (4), (5) for Lorentzian Kac-Moody algebras are also very restricted, but we don't have here so strong results as for hyperbolic root systems (data $(1)-(3))$.

Definition 1.6.1. A lattice $Q$ with two negative squares is called reflective, if its Hermitian symmetric domain $\Omega(Q)$ has a meromorphic (not necessarily holomorphic) automorphic form $\Phi$ of non-zero weight with respect to $G \subset O^{+}(Q)$ of finite index such that its divisor is union of rational quadratic divisors which are orthogonal to some roots of $Q$. The automorphic form $\Phi$ is then also called reflective for $Q$. (Here, for further considerations, we assume that a reflective automorphic form $\Phi$ has non-zero weight, but might be this condition can be weakened by the condition that $\Phi$ is not a constant.)

Obviously, reflectivity of $Q$ does not change by multiplication of the form of the lattice $Q$ by rational numbers.

We suggested in [40, Conjecture 2.2.1]) and [65] 
rk $Q \geq 5$ is finite up to multiplication of forms of lattices $Q$ by positive rational numbers.

We expect the statement of Conjecture 1.6.2 because of Koecher principle (e. g. see [1]): Any non-constant meromorphic automorphic form on a Hermitian symmetric domain $\Omega$ should have non-trivial divisor in $\Omega$ if $\operatorname{dim} \Omega-\operatorname{dim} \Omega_{\infty} \geq$ 2. Here $\Omega_{\infty}$ is the set of points at infinity of $\Omega$ which is added to get Satake compactification $G \backslash \Omega \subset G \backslash\left(\Omega \cup \Omega_{\infty}\right)$ of the arithmetic quotient $G \backslash \Omega$.

For a lattice $Q$ with two negative squares and $G \subset O(Q)$ of a finite index, $\operatorname{dim} \Omega(Q)=\operatorname{rk} Q-2$ and $\operatorname{dim} \Omega(Q)_{\infty}=s(Q)-1$ where $s(Q)$ is the rank of a maximal isotropic sublattice of $Q$. We have $0 \leq s(Q) \leq 2$. In particular, the Koecher principle is valid for $\Omega(Q)$ if rk $Q \geq 5$ or rk $Q \geq 3+s(Q)$.

We apply Koecher principle to restrictions $\Phi \mid \Omega\left(Q_{1}\right)$ of a reflective automorphic form $\Phi$ on all subdomains $\Omega\left(Q_{1}\right) \subset \Omega(Q)$ where $Q_{1} \subset Q$ is a sublattice of $Q$ with two negative squares and rk $Q_{1} \geq 3+s\left(Q_{1}\right)$. Here $\Phi \mid \Omega\left(Q_{1}\right)$ is an automorphic form on the Hermitian symmetric domain $\Omega\left(Q_{1}\right)$ of the same weight as $\Phi$, and it is not a constant, if it is not zero, since its weight is not 0 . Thus, we get

Proposition 1.6.3. Suppose that $Q$ is a reflective lattice with two negative squares. Then

$$
\Omega\left(Q_{1}\right) \bigcap\left(\bigcup_{\text {root } \alpha \in Q} \Omega(Q)_{\alpha}\right) \neq \varnothing
$$

for any sublattice $Q_{1} \subset Q$ such that $Q_{1}$ has two negative squares and rk $Q_{1} \geq$ $3+s\left(Q_{1}\right)$. Here $\Omega(Q)_{\alpha}$ is the rational quadratic divisor which is orthogonal to a root $\alpha \in Q$.

Below we give an example (from [65]) which shows that this condition is very strong.

For a lattice $Q$ any element $\alpha \in Q$ with $\alpha^{2}=2$ is a root. If for the definition of reflective lattices $Q$ and reflective automorphic forms $\Phi$ we shall consider only roots with square 2, we shall get 2-reflective lattices $Q$ and 2-reflective automorphic forms $\Phi$. This is a special case of reflective lattices and reflective automorphic forms.

We consider lattices

$$
T_{n}=H \oplus H \oplus E_{8} \oplus E_{8} \oplus\langle 2 n\rangle
$$

where $n \in \mathbb{N}$. Here $E_{8}$ is an even unimodular positive definite lattice of the rank 8. A lattice $\langle A\rangle$ is the lattice with the matrix $A$ for some basis. We want to show that the lattices $T=T_{n}$ are not 2-reflective for big $n$.

This example is interesting because arithmetic quotients $G \backslash \Omega\left(T_{n}\right)$ by subgroups $G \subset O^{+}\left(T_{n}\right)$ of finite index give moduli of K3 surfaces of degree $2 n$, and

$$
\text { Discr }=\bigcup_{\alpha \in T_{n} \text { with } \alpha^{2}=2} \Omega\left(T_{n}\right)_{\alpha}
$$

is discriminant of the moduli. Points of Discr give K3 surfaces with singularities. Thus, we want to show that the discriminant of K3 surfaces moduli cannot be given as a divisor of an automorphic form of non-zero weight if the degree of K3 surfaces 
Let us consider an even unimodular lattice $L=3 H \oplus E_{8} \oplus E_{8}$. Consider a primitive element $h \in L$ with $h^{2}=-2 n$. Using standard results about indefinite lattices, and discriminant forms technique (see [57]), one can prove that the orthogonal complement $h^{\perp}$ to the element $h$ in $L$ is isomorphic to $T_{n}$.

We use the following general construction. Assume that $K \subset L$ is a primitive sublattice with two negative squares, rk $K \geq 3+s(K)$ where $s(K)$ is the rank of the maximal isotropic sublattice of $K$, and $K$ does not have elements with square 2 . Let $S=K^{\perp}$ be the orthogonal complement to $K$ in $L$. The lattice $S$ is hyperbolic since $L$ has exactly three negative squares.

Let us consider the set $\Delta \subset S^{*}$ with the following properties: if $\delta_{1} \in \Delta$, then (i) $\delta_{1}^{2}>0$; (ii) there exists a $\delta_{2} \in K^{*}$ such that either $\delta_{2}=0$ or $\delta_{2}^{2}>0$, and $\delta_{1}+\delta_{2} \in L$; (iii) $\delta_{1}^{2}+\delta_{2}^{2}=2$. In particular, $0<\delta_{1}^{2} \leq 2$ and $0 \leq \delta_{2}^{2}<2$.

We have a simple

Lemma 1.6.4. Let $h \in S$ is primitive, $h^{2}=-2 n$ and the lattice $h^{\perp} \simeq T_{n}$ is 2-reflective. Then there exists $\delta \in \Delta \subset S^{*}$ such that $h \in \delta^{\perp}$.

Proof. Assume that $T_{n}=h^{\perp}$ is reflective. We apply Proposition 1.6.3 to $Q=T_{n}$, $Q_{1}=K$ and roots with square two. We get that there exists $\delta \in h^{\perp}$ such that $\delta^{2}=2$ and $\Omega\left(h^{\perp}\right)_{\delta} \cap \Omega(K) \neq \emptyset$. We have $\delta=\delta_{1}+\delta_{2}$ where $\delta_{1} \in S^{*}$ and $\delta_{2} \in K^{*}$. It follows that $\delta_{1}^{2}+\delta_{2}^{2}=2$. Since $(h, \delta)=0$ and $h \in S$, it follows that $\left(h, \delta_{1}\right)=0$. The lattice $S$ is hyperbolic and $h^{2}<0$. It follows that either $\delta_{1}^{2}>0$ or $\delta_{1}=0$. The last case is impossible because then $\delta=\delta_{2} \in K$ and $\delta^{2}=2$. But we assume that $K$ does not have elements with square 2. Thus, $\delta_{1}^{2}>0$. If $\delta_{2}=0$, then $\delta_{1} \in \Delta$ and $\left(h, \delta_{1}\right)=0$ as we want. Let $\delta_{2} \neq 0$. Let $\mathbb{C} \omega \in \Omega\left(h^{\perp}\right)_{\delta} \cap \Omega(K)$. Then $\omega \in K \otimes \mathbb{C}$, $(\omega, \omega)=0,(\omega, \bar{\omega})<0$ and $\left(\omega, \delta_{2}\right)=0$. Writing $\omega=a+b i$ where $a, b \in K \otimes \mathbb{R}$, we then get $a^{2}=b^{2}<0$ and $(a, b)=\left(a, \delta_{2}\right)=\left(b, \delta_{2}\right)=0$. Since $K$ has exactly two negative squares, it follows that $\delta_{2}^{2}>0$. It proves the statement.

We can interpret the statement of Lemma 1.6.4 geometrically as follows. Let $\mathcal{L}(S)=V^{+}(S) / \mathbb{R}_{++}$be the hyperbolic space related with the hyperbolic lattice $S$. Each element $\delta \in \Delta$ defines a hyperplane $\mathcal{H}_{\delta} \subset \mathcal{L}(S)$ orthogonal to $\delta$. Elements $\delta \in \Delta$ have $\delta^{2}<2$ and $m \delta \in S$ where $m$ is the exponent of $S^{*} / S$. It follows that the set of hyperplanes $\mathcal{H}_{\delta}, \delta \in \Delta$, is locally finite in $\mathcal{L}(S)$. The lattice $h^{\perp} \simeq T_{n}$ is not reflective if the point $\mathbb{R}_{++} h \in \mathcal{L}(S)$ does not belong to this locally finite set of hyperplanes (one should change $h$ by $-h$ if it is necessary). The set of points $\mathbb{R}_{++} h \in \mathcal{L}(S), h \in S$, is everywhere dense in the hyperbolic space $\mathcal{L}(S)$. There are plenty of these points which do not belong to the locally finite set of hyperplanes $\mathcal{H}_{\delta}, \delta \in \Delta$, and define then non-reflective lattices $h^{\perp} \simeq T_{n}$. For example, it follows that there exists an infinite sequence of integers $n$ such that the lattice $T_{n}$ is not 2-reflective. Exactly that had been demonstrated in [65].

Let us take a concrete lattice $K=H(2) \oplus H(2) \oplus\langle 4\rangle$ where $M(k)$ is a lattice which is obtained from a lattice $M$ by multiplication of the form of the lattice $M$ by $k \in \mathbb{Q}$. Then $S=\langle-4\rangle \oplus D_{8} \oplus D_{8}$ where $D_{8}$ is the root lattice of the root system $\mathbb{D}_{8}$. Thus the lattice $S$ is the set of integral vectors $h=\left(x, u_{1}, \ldots, u_{8}, v_{1}, \ldots, v_{8}\right)$ such that $u_{1}+\cdots+u_{8} \equiv 0 \bmod 2$ and $v_{1}+\cdots+v_{8} \equiv 0 \bmod 2$. The form is given by $-4 x x^{\prime}+u_{1} u_{1}^{\prime}+\cdots+u_{8} u_{8}^{\prime}+v_{1} v_{1}^{\prime}+\cdots+v_{8} v_{8}^{\prime}$.

We consider $h_{0}=(1,0,0, \ldots, 0)$ and $\Delta_{0}=\left\{\delta \in \Delta \mid\left(\delta, h_{0}\right)=0\right\}$ (geometrically, it is the set of hyperplanes $H_{\delta}, \delta \in \Delta$, which contain the point $\left.\mathbb{R}_{++} h_{0}\right)$. We consider 
between points $\mathbb{R}_{++} h_{0}$ and $\mathbb{R}_{++} h$ of the hyperbolic space $\mathcal{L}(S)$ is small enough for the point $\mathbb{R}_{++} h$ would not belong to other hyperplanes $H_{\delta}, \delta \in \Delta$. By Lemma 1.6.4, for these $h$ the lattice $T_{n}=h^{\perp}$ is not 2-reflective. As the result we get

Theorem 1.6.5. Let us consider integers $y$ of the form

$$
y=y_{1}^{2}+y_{2}^{2}+y_{3}^{2}+y_{4}^{2}+y_{5}^{2}+y_{6}^{2}+y_{7}^{2}+y_{8}^{2}
$$

where all $y_{i}$ are natural,

$$
y_{1}+y_{2}+y_{3}+y_{4}+y_{5}+y_{6}+y_{7}+y_{8} \equiv 0 \bmod 2,
$$

and

$$
0<y_{1}<y_{2}<y_{3}<y_{4}<y_{5}<y_{6}<y_{7}<y_{8} .
$$

Let $u$ and $v$ are two numbers of the form (1.6.4) for the corresponding vectors $\left(u_{1}, \ldots, u_{8}\right),\left(v_{1}, \ldots, v_{8}\right)$, the vector $\left(x, u_{1}, \ldots, u_{8}, v_{1}, \ldots, v_{8}\right)$ is primitive (it is sufficient to suppose that it is primitive in the sublattice $u_{1}+\cdots+u_{8} \equiv v_{1}+\cdots+v_{8} \equiv 0$ $\bmod 2)$ and $x^{2}>(9 / 4)(u+v)$. Then for

$$
2 n=4 x^{2}-u-v
$$

the lattice $T_{n}$ is not 2-reflective.

Any sufficiently large even integer $y>N$ can be represented in the form (1.6.4) for some primitive $\left(y_{1}, \ldots, y_{8}\right)$. It follows that any sufficiently large even $2 n$ can be represented in the form (1.6.5), and the lattice $T_{n}$ is not 2-reflective. More exactly, elementary estimates show that it is true for

$$
n>\left(\frac{32}{3}+\sqrt{128+8 N}\right)^{2}
$$

and lattices $T_{n}$ are not 2-reflective for these $n$.

1.7. An example of classification of Lorentzian Kac-Moody algebras or the rank 3. Below and in what follows we consider hyperbolic lattices $S_{t}=$ $H \oplus\langle 2 t\rangle$ and lattices with two negative squares $L_{t}=H \oplus S_{t}=2 H \oplus\langle 2 t\rangle$. We denote

$$
\widehat{O}^{+}\left(L_{t}\right)=\left\{g \in O^{+}\left(L_{t}\right) \mid g \text { is trivial on } L_{t}^{*} / L_{t}\right\} .
$$

The group $\widehat{O}^{+}\left(L_{t}\right)$ is called extended paramodular group. In $\S 2$ below we give classification of Lorentzian Kac-Moody algebras with the root lattice $S_{t}^{*}$, symmetry lattice $L_{t}^{*}$ and the symmetry group $\widehat{O}^{+}\left(L_{t}\right)$.

The lattices $S_{t}^{*}=H \oplus\left\langle\frac{1}{2 t}\right\rangle$ and $L_{t}^{*}=2 H \oplus\left\langle\frac{1}{2 t}\right\rangle$ will be integral after multiplication of their forms by $2 t$. Automorphism groups $O\left(L_{t}\right)=O\left(L_{t}^{*}\right)$ of lattices $L_{t}$ and $L_{t}^{*}$ are naturally identified. Thus, we can consider the group $\widehat{O}^{+}\left(L_{t}\right)$ as a subgroup of $O\left(L_{t}^{*}\right)$.

This case is especially interesting since the lattices $S_{t}$ and $L_{t}$ are maximal even if $t$ is square-free. It follows that many hyperbolic even lattices $S$ of the rank three and many even lattices $L$ of the rank five with two negative squares have equivariant embeddings to $S_{t}$ and $L_{t}$ respectively. Here embedding of lattices $M_{1} \subset M$ of the 
automorphism groups. Any lattice has an equivariant embedding to a maximal one. Thus, studying lattices $S_{t}$ and $L_{t}$, we at the same time study Lorentzian Kac-Moody algebras with root lattices $S^{*}$ and symmetry groups $G \subset O^{+}(L)$ (of finite index) where $S$ has an equivariant embedding to $S_{t}$, and $L$ has an equivariant embedding to $L_{t}$. See (2.2.7) below.

More generally, the same will be true for $m$-dual lattices of lattices $S_{t}$ and $L_{t}$. Here, for a lattice $M$ and a square-free $m \in \mathbb{N}$ the $m$-dual lattice of $M$ is

$$
M^{*, m}=\left(\bigcap_{p \mid m}\left(M \otimes \mathbb{Q} \cap\left(M \otimes \mathbb{Z}_{p}\right)^{*}\right)\right) \bigcap\left(\bigcap_{p \nmid m}\left(M \otimes \mathbb{Q} \cap M \otimes \mathbb{Z}_{p}\right)\right) .
$$

Another importance of this example is its relation with the theory of Abelian surfaces $A$ over $\mathbb{C}$ with polarization of type $(1, t)$. We remind that it means an algebraic integral 2-dimensional cohomology class of $A$ given by a symplectic integral form

$$
J_{t}=\left(\begin{array}{cccc}
0 & 0 & 1 & 0 \\
0 & 0 & 0 & t \\
-1 & 0 & 0 & 0 \\
0 & -t & 0 & 0
\end{array}\right)
$$

in some basis of $H_{1}(A, \mathbb{Z})$. The lattice $\langle 2 t\rangle$ is the Neron-Severi lattice of general Abelian surfaces with polarization of the type $(1, t)$. The lattice $L_{t}(-1)$ is the lattice of transcendental cycles (2-dimensional) of a general Abelian surface with polarization of the type $(1, t)$. The arithmetic quotient $\widehat{O}^{+}\left(L_{t}\right) \backslash \Omega\left(L_{t}\right)$ gives moduli space of Abelian surfaces with polarization of the type $(1, t)$ when one identifies an Abelian surface $A$ with its dual $\widehat{A}$. See [35] and [41] for details. Thus, all automorphic forms with respect to the extended paramodular group $\widehat{O}^{+}\left(L_{t}\right)$ have some geometric interpretation for the moduli space of Abelian surfaces.

\section{Classification of Lorentzian KaC-Moody algebras} WITH THE HYPERBOLIC ROOT LATTICE $S_{t}^{*}$, THE SYMMETRY LATTICE $L_{t}^{*}$, AND THE SYMMETRY GROUP $\widehat{O}^{+}\left(L_{t}\right)$.

Here we give classification (we follow [43]) of Lorentzian Kac-Moody algebras $\mathfrak{g}$ with the root lattice $S_{t}^{*}$, symmetry lattice $L_{t}^{*}$ and the symmetry group $\widehat{O}^{+}\left(L_{t}\right)$. Here $t$ is any natural number. See definitions in Sect. 1.7. Perhaps, this is the first case when a large class of Lorentzian Kac-Moody algebras is classified. We try to remind main definitions and notations from $\S 1$ to make reading easier.

2.1. The formulation of the classification result about Lorentzian KacMoody algebras. By Sect. 1.4, a Lorentzian Kac-Moody algebra $\mathfrak{g}$ with the root lattice $S_{t}^{*}$, the symmetry lattice $L_{t}^{*}$ and the symmetry group $\widehat{O}^{+}\left(L_{t}\right)$ is given by a holomorphic automorphic form $\Phi(z), z \in \Omega\left(V^{+}\left(S_{t}\right)\right)=S_{t} \otimes \mathbb{R}+i V^{+}\left(S_{t}\right)$, with respect to the group $\widehat{O}^{+}\left(L_{t}\right)$ with the Fourier expansion

$$
\Phi(z)=\sum_{w \in W} \varepsilon(w)\left(\exp (-2 \pi i(w(\rho), z))-\sum_{a \in S_{t}^{*} \cap \mathbb{R}_{++} \mathcal{M}} m(a) \exp (-2 \pi i(w(\rho+a), z))\right)
$$

where all coefficients $m(a)$ should be integral; $W \subset O^{+}\left(S_{t}\right)$ is a reflection subgroup 
$W \rightarrow\{ \pm 1\}$ is its quadratic character; $\mathcal{M} \subset \mathcal{L}\left(S_{t}\right)=V^{+}\left(S_{t}\right) / \mathbb{R}_{++}$is a fundamental chamber of $W ; \rho \in S_{t} \otimes \mathbb{Q}$ is the Weyl vector (see (1.4.2)) for the $\operatorname{set} P(\mathcal{M}) \subset S_{t}^{*}$ of orthogonal roots to $\mathcal{M}$ (it is the set of simple real roots of the algebra). Additionally, the automorphic form $\Phi(z)$ should be reflective, i. e. it should have zeros only in rational quadratic divisors which are orthogonal to roots of $L_{t}$. A minor additional condition from Sect. 1.4 is that the semi-direct product $W \rtimes \operatorname{Sym}\left(P(\mathcal{M})_{\overline{1}} \subset P(\mathcal{M})\right)$ should have finite index in $O\left(S_{t}\right)$. Here $P(\mathcal{M})_{\overline{1}} \subset P(\mathcal{M})$ is the set of odd simple real roots defined by the condition that $\varepsilon\left(s_{\alpha}\right)=1$ for the reflection $s_{\alpha}$ with respect to a root $\alpha \in P(\mathcal{M})_{\overline{1}}$. The set $P(\mathcal{M})_{\overline{0}}=P(\mathcal{M})-P(\mathcal{M})_{\overline{1}}$ is the set of even simple real roots.

The automorphic form $\Phi$ automatically has an infinite product expansion

$$
\Phi(z)=\exp (-2 \pi i(\rho, z)) \prod_{\alpha \in \Delta_{+}}(1-\exp (-2 \pi i(\alpha, z)))^{\operatorname{mult}(\alpha)}
$$

where $\operatorname{mult}(\alpha) \in \mathbb{Z}$ are multiplicities of roots of $\mathfrak{g}$ and $\Delta_{+} \subset S_{t}^{*}$ is the set of positive roots of the algebra $\mathfrak{g}$ defined by the condition $\left(\Delta_{+}, \mathcal{M}\right) \leq 0$. The infinite product (2.1.2) can be also used to define the automorphic form $\Phi$ and the Kac-Moody algebra $\mathfrak{g}$. The identity $(2.1 .1)=(2.1 .2)$ is called the denominator identity.

The infinite sum part (2.1.1) of the denominator identity defines generators and defining relations of the Lorentzian Kac-Moody algebra $\mathfrak{g}$, which is a generalized Kac-Moody (or Borcherds) superalgebra. The algebra $\mathfrak{g}$ is graded by the lattice $S_{t}^{*}$

$$
\mathfrak{g}=\bigoplus_{\alpha \in S_{t}^{*}} \mathfrak{g}_{\alpha}=\mathfrak{g}_{0} \bigoplus\left(\bigoplus_{\alpha \in \Delta_{+}} \mathfrak{g}_{\alpha}\right) \bigoplus\left(\bigoplus_{\alpha \in \Delta_{+}} \mathfrak{g}_{-\alpha}\right), \quad \mathfrak{g}_{0}=S_{t} \otimes \mathbb{C}
$$

and the product part (2.1.2) of the denominator identity gives multiplicities

$$
\operatorname{mult}(\alpha):=\operatorname{dim} \mathfrak{g}_{\alpha}=\operatorname{dim} \mathfrak{g}_{\alpha, \overline{0}}-\operatorname{dim} \mathfrak{g}_{\alpha, \overline{1}}
$$

of root-spaces $\mathfrak{g}_{\alpha}, \alpha \in \Delta_{+}$, of the superalgebra $\mathfrak{g}$. We also have $\operatorname{mult}(-\alpha)=$ $\operatorname{mult}(\alpha)$.

Our classification result is

Theorem 2.1.1. There are exactly 29 Lorentzian Kac-Moody algebras $\mathfrak{g}$ with the root lattice $S_{t}^{*}$, the symmetry lattice $L_{t}^{*}$ and the symmetry group $\widehat{O}^{+}\left(L_{t}\right)$ (equivalently, there are exactly 29 automorphic forms (2.1.1)) for all natural $t \in \mathbb{N}$. They exist only for

$$
\begin{aligned}
t= & 1 \text { (three), } 2(\text { seven }), 3 \text { (seven }), 4(\text { seven }), 8 \text { (one }), 9 \text { (one }), \\
& 12 \text { (one), } 16 \text { (one), } 36 \text { (one). }
\end{aligned}
$$

where for each $t$ we show in brackets the number of forms. All these 29 forms are given in Sect. 2.6 below.

The proof of Theorem 2.1.1 is divided in two parts. First, one should construct all 29 automorphic forms of the theorem. Second, one should prove that there are no 
the 29 automorphic forms in Sect. 2.2. The completeness of their list is considered in Sects $2.2-2.4$.

All 29 automorphic forms of Theorem 2.1.1 were found in [36], [37], [39], [41] together with their infinite sum and product expansions. Formally, the forms for $t=8,12,16$ are new but they coincide with some forms for $t=2,3,4$ respectively after appropriate change of variables. Thus, in fact, they are not new.

We describe all 29 automorphic forms of Theorem 2.1.1 in Sect. 2.6 below. To construct these automorphic forms, we used in [41] a variant of the Borcherds exponential lifting [7] which we applied to Jacobi modular forms, see [41, Theorem 2.1]. It gives the infinite product expansion (2.1.2) of the forms, and construction of these forms using the infinite product expansion. We consider this variant in the next Sect. 2.2.

To find Fourier expansion of the 29 automorphic forms, we have used in [36], [37], [39], [41] several different methods, there are no general methods for that. One of these methods is arithmetic lifting from [29] - [32] of Jacobi forms which gives simple Fourier expansions (2.1.1) of some of the 29 automorphic forms. In this paper we don't discuss the methods of construction of the Fourier expansions of the 29 automorphic forms, and only give the corresponding formulae. We don't need these Fourier expansions for the proof of Theorem 2.1.1, but they are very important for the construction of the algebras $\mathfrak{g}$ using generators and defining relations.

2.2. A variant for Jacobi forms of Borcherds automorphic products. We use a general result from [41] which permits to construct as products similar to (2.1.2) many automorphic forms with respect to the extended paramodular group.

We use a basis $f_{2}, f_{-2}$ for $H$ with the Gram matrix $\left(\begin{array}{cc}0 & -1 \\ -1 & 0\end{array}\right)$ and $f_{3}$ for $\langle 2 t\rangle$. Together they give a basis $f_{2}, f_{3}, f_{-2}$ for the lattice $S_{t}=H \oplus\langle 2 t\rangle$ with the Gram $\operatorname{matrix}\left(\begin{array}{ccc}0 & 0 & -1 \\ 0 & 2 t & 0 \\ -1 & 0 & 0\end{array}\right)$. The dual lattice $S_{t}^{*}$ has the basis $f_{2}, \widehat{f_{3}}=f_{3} / 2 t, f_{-2}$. We denote $\alpha=(n, l, m):=n f_{2}-l \widehat{f_{3}}+m f_{-2} \in S_{t}^{*}$, where $\alpha^{2}=-2 n m+\frac{l^{2}}{2 t}$. We denote by $D(\alpha)=2 t \alpha^{2}=-4 t n m+l^{2}$ the discriminant or norm of $\alpha$. For the dual lattice $S_{t}^{*}$ we usually use this form. Thus, the discriminant or the norm gives the integral lattice $S_{t}^{*}(2 t)$. Let $z=z_{3} f_{2}+z_{2} f_{3}+z_{1} f_{-2} \in \Omega\left(V^{+}\left(S_{t}\right)\right)$. Then $\exp (-2 \pi i(\alpha, z))=q^{n} r^{l} s^{m}$ where $q=\exp \left(2 \pi i z_{1}\right), r=\exp \left(2 \pi i z_{2}\right), s=\exp \left(2 \pi i z_{3}\right)$.

In [41] a variant of the Borcherds exponential lifting was proved. Borcherds exponential lifting [7] gives lifting of modular forms of one variable. In [41] we constructed its variant for Jacobi modular forms. We formulate that in Theorem 2.2.1 below. See Sect. 3 for definitions and results about Jacobi modular forms which we need.

Let

$$
\phi_{0, t}(\tau, z)=\sum_{k, l \in \mathbb{Z}} f(k, l) q^{k} r^{l} \in J_{0, t}^{n h}
$$

be a nearly holomorphic Jacobi form of weight 0 and index $t \in \mathbb{N}$ (i.e. $k$ might be negative for the Fourier expansion), where $q=\exp (2 \pi i \tau), \operatorname{Im} \tau>0, r=\exp (2 \pi i z)$, $z \in \mathbb{C}$. It is automorphic with respect to the Jacobi group $H(\mathbb{Z}) \rtimes S L_{2}(\mathbb{Z})$ where $H(\mathbb{Z})$ is the integral Heisenberg group which is the central extension 
Nearly holomorphic means that the form $\phi_{0, t}$ is holomorphic except a possible pole of a finite order at infinity $q=0$. The Fourier coefficient $f(k, l)$ of $\phi_{0, t}$ depends only on the norm $4 t k-l^{2}$ of $(k, l)$ and $l \bmod 2 t$. Moreover, $f(k, l)=f(k,-l)$. From the definition of nearly holomorphic forms, it follows that the norm $4 t k-l^{2}$ of indices of non-zero Fourier coefficients $f(k, l)$ are bounded from bellow.

Let

$$
\phi_{0, t}^{(0)}(z)=\sum_{l \in \mathbb{Z}} f(0, l) r^{l}
$$

be the $q^{0}$-part of $\phi_{0, t}(\tau, z)$. Its Fourier coefficients are especially important for the Theorem below.

Theorem 2.2.1 [41, Theorem 2.1]. Assume that $t \in \mathbb{N}$ and the Fourier coefficients $f(k, l)$ of a Jacobi form $\phi_{0, t}$ from (2.2.1) are integral. Then the infinite product

$$
B_{\phi}(z)=q^{A} r^{B} s^{C} \prod_{\substack{n, l, m \in \mathbb{Z} \\(n, l, m)>0}}\left(1-q^{n} r^{l} s^{m}\right)^{f(n m, l)}
$$

where

$$
A=\frac{1}{24} \sum_{l} f(0, l), \quad B=\frac{1}{2} \sum_{l>0} l f(0, l), \quad C=\frac{1}{4 t} \sum_{l} l^{2} f(0, l),
$$

and $(n, l, m)>0$ means that either $m>0$ or $m=0$ and $n>0$ or $m=n=0$ and $l<0$, defines a meromorphic automorphic form of weight $\frac{f(0,0)}{2}$ with respect to $\widehat{O}^{+}\left(L_{t}\right)$ with a character (or a multiplier system if the weight is half-integral). All components of the divisor of $B_{\phi}(z)$ are the rational quadratic divisors orthogonal to $\alpha=(a, b, 1)$ of the discriminant $D=-4 t a+b^{2}>0$ (up to the action of $\widehat{O}^{+}\left(L_{t}\right)$ ) with multiplicities

$$
m_{D, b}=\sum_{n>0} f\left(n^{2} a, n b\right)
$$

See other details in [41, Theorem 2.1].

All 29 automorphic forms of Theorem 2.1.1 are given by some automorphic products of Theorem 2.2.1. It is how we call all automorphic forms of Theorem 2.2.1. Thus, to give all these 29 automorphic forms, we should give the corresponding 29 Jacobi forms. We give all these forms in Sect. 2.6, Table 2.

More generally, we describe all reflective meromorphic automorphic forms which are given by the Theorem 2.2.1. Here a meromorphic automorphic form on the domain $\Omega\left(L_{t}\right)$ is called reflective if its divisor is a sum with some multiplicities of rational quadratic divisors orthogonal to roots of $L_{t}$. We remind that an element $\alpha \in L_{t}$ is called root if $\alpha^{2}>0$ and $\alpha^{2} \mid 2\left(\alpha, L_{t}\right)$. Using description of the divisor of $B_{\phi}$ in Theorem 2.2.1, it is easy to prove

Lemma 2.2.2. An infinite product $B_{\phi}$ given by a Jacobi form $\phi=\phi_{0, t}$ with integral Fourier coefficients (from Theorem 2.2.1) defines a reflective automorphic form if and only if each non-zero Fourier coefficient $f(k, l)$ of $\phi_{0, t}$ with negative norm $4 t k-l^{2}<0$ satisfies 
Let us denote by $R J_{t}$ the space of all Jacobi forms $\phi=\phi_{0, t}$ of the index $t$ of Theorem 2.2.1 which give reflective automorphic products $B_{\phi}$ (equivalently, $\phi=$ $\phi_{0, t}$ satisfies Lemma 2.2.2). It is natural to call these Jacobi forms reflective either. The space $R J_{t}$ of all reflective Jacobi forms is a free $\mathbb{Z}$-module with respect to addition. We have

Main Theorem 2.2.3. For $t \in \mathbb{N}$ the space $R J_{t}$ of reflective Jacobi forms of the index $t$ is not trivial (i.e. it is not equal to zero) if and only if $t$ is equal to

$$
\begin{aligned}
& \text { 1(2), 2(3), 3(3), 4(3), 5(3), 6(4), 7(2), 8(3), 9(3), 10(3), 11(1), 12(4), 13(2), } \\
& \text { 14(3), 15(2), 16(2), 17(1), 18(3), 20(3), 21(3), 22(1), 24(2), 25(1), 26(1), } \\
& 28(1), 30(3), 33(1), 34(2), 36(3), 39(2), 42(1), 45(1), 48(1), 63(1), 66(1) .
\end{aligned}
$$

where in brackets we show the rank of the corresponding $\mathbb{Z}$-module $R J_{t}$ of reflective Jacobi forms.

In Table 1 of Sect. 2.5 below we give a basis of the $\mathbb{Z}$-module $R J_{t}$ for $t=1,2,3$, 4, 8, 9, 12, 16, 36 when the subspace $R J_{t}$ also contains a Jacobi form which gives the denominator identity for a Lorentzian Kac-Moody algebra (i.e. it gives some forms of Theorem 2.1.1).

All automorphic forms of Theorem 2.1.1 have the property that they have multiplicity 1 for components of their divisors. This follows from properties of real roots of $\mathfrak{g}$ and the infinite product decomposition (2.1.2). If a simple real root $\alpha \in P(\mathcal{M})$ of $\mathfrak{g}$ is even (i. e. $\alpha \in P(\mathcal{M})_{\overline{0}}$ ), then $m \alpha, m \in \mathbb{N}$, is a root of $\mathfrak{g}$ if and only if $m=1$, and multiplicity of the root $\alpha$ is one. If a simple real root $\alpha \in P(\mathcal{M})$ is odd, then $m \alpha, m \in \mathbb{N}$, is a root if and only if $m=1$ or $m=2$. The root $\alpha$ has multiplicity $(-1)$, and the root $2 \alpha$ has multiplicity 1 . From the full list of reflective Jacobi forms of Main Theorem 2.2.3, it is not hard to find all Jacobi forms $\phi$ with the divisor of multiplicity one for $B_{\phi}$, since Theorem 2.2.1 gives multiplicities of divisors of its automorphic products. See Table 1 of Sect. 2.5 for $t=1,2,3,4,8$, $9,12,16,36$. For all other $t$ the list of all reflective Jacobi forms of Theorem 2.2.3 will be given in a forthcoming publication. [44]. It is too long to be presented here.

Potentially, Main Theorem 2.2.3 contains information about all reflective automorphic forms with infinite product expansion of the type of Theorem 2.2.1 for all equivariant sublattices $L \subset L_{t}$ of finite index. Here equivariant means that $O(L) \subset O\left(L_{t}\right)$; in particular, every root of $L$ is multiple to a root of $L_{t}$. If $L$ has a reflective automorphic form $\Phi$ with respect to $O(L)$ with an infinite product expansion, then its symmetrization

$$
\prod_{g \in O(L) \backslash O\left(L_{t}\right)} g^{*} \Phi
$$

is a reflective automorphic form with an infinite product expansion for the lattice $L_{t}$.

Thus, potentially, Main Theorem 2.2.3 contains important information about reflective automorphic forms with infinite products and about automorphic forms of denominator identities of Lorentzian Kac-Moody algebras with the symmetry lattices $L^{*}$ instead of $L_{t}^{*}$ and the corresponding hyperbolic root lattices $S^{*}$ where $S=S_{t} \cap L$ instead of $S_{t}$. Moreover, one can possibly consider more general class of Lie algebras for which reflective forms of Main Theorem 2.2.3 may give kind of 
2.3. The proof of Main Theorem 2.2.3 and reflective hyperbolic lattices. To classify finite-dimensional semi-simple and affine Kac-Moody algebras, one needs to classify corresponding finite and affine root systems. To prove Main Theorem 2.2.3, one needs description of appropriate hyperbolic root systems. They are root subsystems of reflective hyperbolic lattices which we have considered in Sect. 1.5 .

Let $S$ be a hyperbolic (i.e. of the signature $(m, 1)$ ) lattice, $W(S)$ its reflection group and $\mathcal{M} \subset V^{+}(S) / \mathbb{R}_{++}$its fundamental chamber, and $\operatorname{Sym}(\mathcal{M})$ is the symmetry group of the fundamental chamber. Thus, we have the corresponding semi-direct product $O^{+}(S)=W(S) \rtimes S y m(\mathcal{M})$. We remind (see Sect. 1.5) that $S$ is called reflective if $\mathcal{M}$ has a generalized Weyl vector $\rho \in S \otimes \mathbb{Q}$. It means that $\rho \neq 0$ and the orbit $\operatorname{Sym}(\mathcal{M})(\rho)$ is finite. A reflective lattice is called elliptically reflective if it has a generalized Weyl vector $\rho$ with $\rho^{2}<0$. It is called parabolically reflective if it is not elliptically reflective but has a generalized Well vector $\rho$ with $\rho^{2}=0$. It is called hyperbolically reflective if it is not elliptically or parabolically reflective, but it has a generalized Weyl vector $\rho$ with $\rho^{2}>0$.

Suppose that $B_{\phi}(z)$ is an automorphic form of Theorem 2.2.1 which is reflective. The inequality $(n, l, m)>0$ of Theorem 2.2.1 is a variant of choosing a fundamental chamber $\mathcal{M}$ of $W\left(S_{t}\right)$. The vector $\rho=(A, B, C)$ is invariant with respect to the group $\widehat{\operatorname{Sym}}(\mathcal{M})=\operatorname{Sym}(\mathcal{M}) \cap \widehat{O}^{+}\left(L_{t}\right)$ which has finite index in $\operatorname{Sym}(\mathcal{M})$. If $\rho=(A, B, C)$ is not zero, it then defines a generalized Weyl vector for $\operatorname{Sym}(\mathcal{M})$. If the form $B_{\phi}(z)$ has a zero vector $\rho=(A, B, C)$, one can change $B_{\phi}(z)$ by other form $w^{*}\left(B_{\phi}(z)\right)$ where $w \in W$ is an appropriate reflection, in such a way that $w^{*}\left(B_{\phi}(z)\right)$ will have a non-zero generalized Weyl vector $\rho$. Thus, we get

Lemma 2.3.1. If the space $R J_{t}$ of reflective Jacobi forms is not zero, then the lattice $S_{t}$ is reflective.

It is interesting that the space $R J_{t}$ may really have a Jacobi form with zero vector $\rho=(A, B, C)$. It happens for $t=6$ and $t=12$ when $\operatorname{rk} R J_{t}=4$.

The monograph [68] was devoted to classification of reflective hyperbolic lattices and appropriate (for Lorentzian Kac-Moody algebras) hyperbolic root systems of the rank three. This classification contains 122 main elliptic types and 66 main hyperbolic types (there are no main parabolic types). In particular, all reflective hyperbolic lattices of the rank three with square-free determinant were classified (e. g. it gives classification of all reflective lattices $S_{t}$ when $t$ is square-free, see Theorem 2.3.2 below). It follows classification of all maximal reflective hyperbolic lattices of rank three. For arbitrary reflective hyperbolic lattices of rank three, effective methods of their enumeration and effective estimates of their invariants were obtained.

Using these results and direct calculations, we get

Theorem 2.3.2. The lattice $S_{t}=H \oplus\langle 2 t\rangle$ is reflective for the following and the 
(e) for elliptic, (p) for parabolic and (h) for the hyperbolic type:

$$
\begin{aligned}
t= & 1-22(e), 23(h), 24-26(e), 28(e), 29(h), 30(e), 31(h), \\
& 33(e), 34(e), 35(h), 36(e), 37(h), 38(h), 39(e), 40(h), 42(e), \\
& 44(h), 45(e), 46(h), 48(h), 49(e), 50(e), 52(e), 55(e), 56(h), \\
& 57(h), 60(h), 63(h), 66(e), 70(h), 72(h), 78(h), 84(h), 90(h), \\
& 100(h), 105(h) .
\end{aligned}
$$

In particular, there are no reflective lattices $S_{t}$ of parabolic type.

To prove Main Theorem 2.2.3 and to find a basis of $R J_{t}$, one needs to analyze only the $t$ of Theorem 2.3.2. In particular, $t \leq 105$. To find the rank of $R J_{t}$ and to find a basis of $R J_{t}$, one can use known generators of the graded ring of weak Jacobi forms. Over $\mathbb{Q}$ (with rational Fourier coefficients) these generators were found in [24] (see Sect. 3), and this is sufficient for calculation of $\mathrm{rk} R J_{t}$ (e. g. one can use computer). Generators of the graded ring of weak Jacobi forms with integral Fourier coefficients were found in [33] and [34] (see Sect. 3). There results permit to find basis of $R J_{t}$ for all $t$ of Theorem 2.2.3. We give it for $t=1,2,3,4,8,9,12$, 16, 36 in Table 1 of Sect. 2.5 below. To simplify these calculations, one can also use arguments which we give below for the proof of Theorem 2.1.1.

Classification of all reflective hyperbolic lattices of rank three in [68] gives a hope that all Lorentzian Kac-Moody algebras of the rank three (with arbitrary hyperbolic root lattice $S$ of the rank three) can be classified.

Finiteness results for all reflective hyperbolic lattices (Theorems 1.5.3 and 1.5.5) and the reasonable number of main types for reflective hyperbolic lattices of the rank three $(122+66)$ give a hope that all Lorentzian Kac-Moody algebras of rank $\geq 3$ can be classified in a future. We expect that number of cases drops when the rank is increasing. We think that the rank three case is the most reach and complicated.

2.4. Proof of Theorem 2.1.1 and reflective hyperbolic lattices with a Weyl vector. Here we sketch the proof of Theorem 2.1.1 to emphasize importance of reflective hyperbolic lattices and hyperbolic root systems with a Weyl vector.

Using Theorem 2.2.3 and considerations above, we have proved that Theorem 2.1.1 gives all automorphic forms (satisfying its conditions) which can be obtained by the general construction of Theorem 2.2.1. Here we want to show that there are no other automorphic forms satisfying conditions of Theorem 2.1.1. Thus, any of forms of Theorem 2.1.1 can be obtained by the construction of Theorem 2.2.1.

For Theorem 2.1.1, the fundamental polyhedron $\mathcal{M}$ and the set $P(\mathcal{M})$ of orthogonal roots to $\mathcal{M}$ have a Weyl vector $\rho$ (satisfying (1.4.2)). The $P(\mathcal{M})$ and $\rho$ are invariant with respect to the group $\widehat{\operatorname{Sym}}(P(\mathcal{M}))$ which has finite index in $\operatorname{Sym}(\mathcal{M})$ (we use notation $\widehat{G}=G \cap \widehat{O}^{+}\left(L_{t}\right)$ ). In particular, the corresponding lattices $S_{t}$ are reflective. For any reflective lattice $S_{t}$ (it belongs to the list of Theorem 2.3.2) the fundamental chamber $\mathcal{M}_{0}$ for the full reflection group $W\left(S_{t}\right)$ can be calculated and is known (for $t=1,2,3,4,8,9,12,16,36$ these calculations are presented in Table 1 below). Thus, the fundamental chamber $\mathcal{M}$ is composed from the known polygon $\mathcal{M}_{0}$ by some reflections, and it has a Weyl vector $\rho$. Using this information, we can find all possible $\mathcal{M}, P(\mathcal{M}), \rho$ and predict the divisor of the reflective automorphic 
of them (for the corresponding $t$ ) has the same divisor. By Koecher principle (we discussed this principle in Sect. 1.6), the form $\Phi(z)$ is equal to that form.

Similar arguments can be used to classify all reflective meromorphic automorphic forms with infinite product of the type (2.1.2) and with a generalized Weyl vector $\rho \neq 0$. But we don't require that multiplicities of the infinite product are related with Fourier coefficients of any modular form. Like the product (2.1.2), this product should be related with a reflection subgroup $W \subset W\left(S_{t}\right)$, its fundamental chamber $\mathcal{M}$, the set $P(\mathcal{M}) \subset S_{t}^{*}$ of orthogonal roots to $\mathcal{M}$ (they define $\Delta_{+} \subset S_{t}^{*}$ ), and a generalized Weyl vector $\rho \in S_{t} \otimes \mathbb{Q}$ (i. e. $\rho \neq 0$, the orbit $\operatorname{Sym}(P(\mathcal{M}))(\rho)$ is finite and $W \rtimes \operatorname{Sym}(P(\mathcal{M}))$ has finite index in $\left.O\left(S_{t}\right)\right)$. The function mult $(\alpha), \alpha \in \Delta_{+}$, should be integral and invariant with respect to $\widehat{\operatorname{Sym}}(P(\mathcal{M}))$. The product should converge in a neighborhood $i m(z)^{2}<<0$ of the cusp at infinity. All the definitions are similar. We have

Theorem 2.4.1. Any reflective meromorphic automorphic form with the root lattice $S_{t}^{*}$, the symmetry lattice $L_{t}^{*}$ and the symmetry group $\widehat{O}^{+}\left(L_{t}\right)$ having infinite product of the type (2.1.2) with a non-zero generalized Weyl vector $\rho$ is $B_{\phi}$ where $\phi$ belongs to the list of Main Theorem 2.2.3.

Applying to the forms of Theorem 2.4.1 reflections with respect to roots in $S_{t}$, one can get some automorphic forms with zero generalized Weyl vector and with infinite product. They appear only for $t=6$ and $t=12$.

2.5. Reflective Jacobi forms from $R J_{t}$ for $t=1,2,3,4,8,9,12,16,36$. For these $t$ we give the basis $\xi_{0, t}^{(1)}, \ldots \xi_{0, t}^{(r k)}$ of the $\mathbb{Z}$-module $R J_{t}$. For each Jacobi form of the basis we show the leading part of its Fourier expansion which defines the Jacobi form uniquely. We give all its Fourier coefficients with negative norm (up to equivalence); the corresponding negative norm is shown in brackets [.]. We also give a formula for the form which uses basic Jacobi forms. In these formulae $E_{4}=E_{4}(\tau)$ and $\Delta_{12}=\Delta(\tau)$ are the Eisenstein series of weight 4 and the Ramanujan function of weight 12 for $S L_{2}(\mathbb{Z})$ respectively, $E_{4, m}(m=1,2,3)$ are Eisenstein-Jacobi series of weight 4 and index $m$ (see [24]), and $\phi_{0,1}, \phi_{0,2}, \phi_{0,3}, \phi_{0,4}$ are the four generators from [33] and [41] of the graded ring of the weak Jacobi forms of weight zero with integral Fourier coefficients. See Sect. 3 about these Jacobi forms.

We give the set $\bar{R}$ of primitive roots in $S_{t}^{*}$ up to equivalence (up to the action of the group $\left.\pm \widehat{O}\left(L_{t}\right)\right)$. Up to this equivalence, a root $\alpha=(n, l, m)$ is defined by its norm $-2 t \alpha^{2}=-4 n m+l^{2}$ and $\pm l \bmod 2 t$. We also give the matrix

$$
\operatorname{Mul}(\bar{R}, \xi)=\operatorname{mul}\left(\gamma_{i}, \xi_{0, t}^{(j)}\right)
$$

where $\operatorname{mul}\left(\gamma_{i}, \xi_{0, t}^{(j)}\right)$ is the multiplicity of the form $\Phi_{\xi_{0, t}^{(j)}}$ in the rational quadratic divisor which is orthogonal to the root from the equivalence class $\gamma_{i} \in \bar{R}$.

We give the set $P\left(\mathcal{M}_{0}\right)$ of primitive roots in $S_{t}^{*}$ which is orthogonal to the fundamental chamber $\mathcal{M}_{0}$ of the reflection group $W\left(S_{t}\right)$ (this is equivalent to the ordering $(n, l, m)>0$ used in Theorem 2.2.1), and its Gram matrix

$$
G\left(P\left(\mathcal{M}_{0}\right)\right)=2 t((\alpha, \beta)), \alpha, \beta \in P\left(\mathcal{M}_{0}\right) .
$$

Thus, we identify the dual lattice $S_{t}^{*}$ with the integral lattice $S_{t}^{*}(2 t)=H(2 t) \oplus\langle 1\rangle$ 
All these data are given in Table 1 below.

Table 1. Basis of the space $R J_{t}$ of reflective

Jacobi forms for $t=1,2,3,4,8,9,12,16,36$.

Case $t=1$. The space $R J_{1}$ has the basis

$$
\begin{aligned}
\xi_{0,1}^{(1)} & =\phi_{0,1}=\left(r[-1]+10+r^{-1}[-1]\right)+O(q) ; \\
\xi_{0,1}^{(2)} & =E_{4}{ }^{2} E_{4,1} / \Delta_{12}-57 \phi_{0,1} \\
& =q^{-1}[-4]+\left(r^{2}[-4]-r[-1]+60-r^{-1}[-1]+r^{-2}[-4]\right)+O(q) .
\end{aligned}
$$

We have $\bar{R}=\overline{P\left(\mathcal{M}_{0}\right)}$ and

$$
\begin{gathered}
P\left(\mathcal{M}_{0}\right)=\left(\begin{array}{ccc}
1 & 2 & 0 \\
0 & -1 & 0 \\
-1 & 0 & 1
\end{array}\right) \equiv\left[\begin{array}{c}
4, \overline{0} \\
1, \overline{1} \\
4, \overline{0}
\end{array}\right] ; \quad \operatorname{Mul}\left(P\left(\mathcal{M}_{0}\right), \xi\right)=\left(\begin{array}{ll}
0 & 1 \\
1 & 0 \\
0 & 1
\end{array}\right) ; \\
G\left(P\left(\mathcal{M}_{0}\right)\right)=\left(\begin{array}{ccc}
4 & -2 & -2 \\
-2 & 1 & 0 \\
-2 & 0 & 4
\end{array}\right) .
\end{gathered}
$$

Case $t=2$. The space $R J_{2}$ has the basis

$$
\begin{aligned}
& \xi_{0,2}^{(1)}=\phi_{0,2}=\left(r[-1]+4+r^{-1}[-1]\right)+O(q) ; \\
& \xi_{0,2}^{(2)}=\left(\phi_{0,1}\right)^{2}-21 \phi_{0,2}=\left(r^{2}[-4]-r[-1]+18-r^{-1}[-1]+r^{-2}[-4]\right)+O(q) ; \\
& \xi_{0,2}^{(3)}=E_{4}{ }^{2} E_{4,2} / \Delta_{12}-14\left(\phi_{0,1}\right)^{2}+216 \phi_{0,2}=q^{-1}[-8]+24+O(q) .
\end{aligned}
$$

We have $\bar{R}=\overline{P\left(\mathcal{M}_{0}\right)}$ where

$$
\begin{gathered}
P\left(\mathcal{M}_{0}\right)=\left(\begin{array}{ccc}
1 & 2 & 0 \\
0 & -1 & 0 \\
-1 & 0 & 1
\end{array}\right) \equiv\left[\begin{array}{l}
4, \overline{2} \\
1, \overline{1} \\
8, \overline{0}
\end{array}\right] ; \quad \operatorname{Mul}\left(P\left(\mathcal{M}_{0}\right), \xi\right)=\left(\begin{array}{lll}
0 & 1 & 0 \\
1 & 0 & 0 \\
0 & 0 & 1
\end{array}\right) ; \\
G\left(P\left(\mathcal{M}_{0}\right)\right)=\left(\begin{array}{ccc}
4 & -2 & -4 \\
-2 & 1 & 0 \\
-4 & 0 & 8
\end{array}\right) .
\end{gathered}
$$

Case $t=3$. The space $R J_{3}$ has the basis

$\xi_{0,3}^{(1)}=\phi_{0,3}=\left(r[-1]+2+r^{-1}[-1]\right)+O(q) ;$

$\xi_{0,3}^{(2)}=\phi_{0,1} \phi_{0,2}-15 \phi_{0,3}=\left(r^{2}[-4]-r[-1]+12-r^{-1}[-1]+r^{-2}[-4]\right)+O(q)$; 
We have $\bar{R}=\overline{P\left(\mathcal{M}_{0}\right)}$ where

$$
\begin{gathered}
P\left(\mathcal{M}_{0}\right)=\left(\begin{array}{ccc}
1 & 2 & 0 \\
0 & -1 & 0 \\
-1 & 0 & 1
\end{array}\right) \equiv\left[\begin{array}{c}
4, \overline{2} \\
1, \overline{1} \\
12, \overline{0}
\end{array}\right] ; \quad \operatorname{Mul}\left(P\left(\mathcal{M}_{0}\right), \xi\right)=\left(\begin{array}{lll}
0 & 1 & 0 \\
1 & 0 & 0 \\
0 & 0 & 1
\end{array}\right) ; \\
G\left(P\left(\mathcal{M}_{0}\right)\right)=\left(\begin{array}{ccc}
4 & -2 & -6 \\
-2 & 1 & 0 \\
-6 & 0 & 12
\end{array}\right) .
\end{gathered}
$$

Case $t=4$. The space $R J_{4}$ has the basis

$$
\begin{aligned}
\xi_{0,4}^{(1)} & =\phi_{0,4}=\left(r[-1]+1+r^{-1}[-1]\right)+O(q) \\
\xi_{0,4}^{(2)} & =\left(\phi_{0,2}\right)^{2}-9 \phi_{0,4}=\left(r^{2}[-4]-r[-1]+9-r^{-1}[-1]+r^{-2}[-4]\right)+O(q) \\
\xi_{0,4}^{(3)} & =E_{4} E_{4,1} E_{4,3} / \Delta_{12}-2\left(\phi_{0,1}\right)^{2} \phi_{0,2}+20 \phi_{0,1} \phi_{0,3}+16 \phi_{0,4} \\
& =q^{-1}[-16]+24+O(q) .
\end{aligned}
$$

We have $\bar{R}=\overline{P\left(\mathcal{M}_{0}\right)}$ where

$$
\begin{gathered}
P\left(\mathcal{M}_{0}\right)=\left(\begin{array}{ccc}
1 & 2 & 0 \\
0 & -1 & 0 \\
-1 & 0 & 1
\end{array}\right) \equiv\left[\begin{array}{c}
4, \overline{2} \\
1, \overline{1} \\
16, \overline{0}
\end{array}\right] ; \quad \operatorname{Mul}\left(P\left(\mathcal{M}_{0}\right), \xi\right)=\left(\begin{array}{lll}
0 & 1 & 0 \\
1 & 0 & 0 \\
0 & 0 & 1
\end{array}\right) ; \\
G\left(P\left(\mathcal{M}_{0}\right)\right)=\left(\begin{array}{ccc}
4 & -2 & -8 \\
-2 & 1 & 0 \\
-8 & 0 & 16
\end{array}\right) .
\end{gathered}
$$

Case $t=8$. The space $R J_{8}$ has the basis

$$
\begin{aligned}
\xi_{0,8}^{(1)} & =\left(\phi_{0,2}\right)^{2} \phi_{0,4}-\phi_{0,2}\left(\phi_{0,3}\right)^{2}-\left(\phi_{0,4}\right)^{2}=\left(2 r[-1]-1+2 r^{-1}[-1]\right) \\
& +\left(-r^{6}[-4]-2 r^{5}+4 r^{4}-4 r^{3}+r^{2}+6 r-8+\cdots\right) q+O\left(q^{2}\right) ; \\
\xi_{0,8}^{(2)} & =\phi_{0,2}(\tau, 2 z)=\phi_{0,1} \phi_{0,3} \phi_{0,4}+\phi_{0,2}\left(\phi_{0,3}\right)^{2}-2\left(\phi_{0,2}\right)^{2} \phi_{0,4}-2\left(\phi_{0,4}\right)^{2} \\
& =\left(r^{2}[-4]+4+r^{-2}[-4]\right)+\left(r^{6}[-4]-8 r^{4}-r^{2}+16-\cdots\right) q+O\left(q^{2}\right) ; \\
\xi_{0,8}^{(3)} & =E_{4} E_{4,3}\left(E_{4,2} \phi_{0,3}-E_{4,1} \phi_{0,4}\right) / \Delta_{12}-3\left(\phi_{0,1}\right)^{2}\left(\phi_{0,3}\right)^{2}+2\left(\phi_{0,1}\right)^{2} \phi_{0,2} \phi_{0,4} \\
& +\phi_{0,1} \phi_{0,3} \phi_{0,4}-16\left(\phi_{0,4}\right)^{2}=q^{-1}[-32]+24+\left(8 r^{6}[-4]+256 r^{5}+2268 r^{4}\right. \\
& \left.+9472 r^{3}+23608 r^{2}+39424 r+46812+\cdots\right) q+O\left(q^{2}\right) .
\end{aligned}
$$

We have $\bar{R}=\overline{P\left(\mathcal{M}_{0}\right)}$ where

$$
P\left(\mathcal{M}_{0}\right)=\left(\begin{array}{ccc}
1 & 2 & 0 \\
0 & -1 & 0 \\
-1 & 0 & 1
\end{array}\right) \equiv\left[\begin{array}{c}
4, \overline{2} \\
1, \overline{1} \\
32, \overline{0}
\end{array}\right] ; \quad \operatorname{Mul}\left(P\left(\mathcal{M}_{0}\right), \xi\right)=\left(\begin{array}{lll}
0 & 1 & 0 \\
2 & 1 & 0 \\
0 & 0 & 1
\end{array}\right)
$$




$$
G\left(P\left(\mathcal{M}_{0}\right)\right)=\left(\begin{array}{cccc}
4 & -2 & -16 & -4 \\
-2 & 1 & 0 & -6 \\
-16 & 0 & 32 & 0 \\
-4 & -6 & 0 & 4
\end{array}\right)
$$

Case $t=9$. The space $R J_{9}$ has the basis

$$
\begin{aligned}
\xi_{0,9}^{(1)} & =-\phi_{0,1}\left(\phi_{0,4}\right)^{2}+6 \phi_{0,2} \phi_{0,3} \phi_{0,4}-5\left(\phi_{0,3}\right)^{3}=\left(3 r[-1]-2+3 r^{-1}[-1]\right) \\
& +\left(-4 r^{6}+6 r^{5}-12 r^{4}+22 r^{3}-30 r^{2}+36 r-36+\cdots\right) q+\left(-r^{9}[-9]-6 r^{8}\right. \\
& \left.+15 r^{7}-36 r^{6}+72 r^{5}-120 r^{4}+171 r^{3}-216 r^{2}+255 r-268+\cdots\right) q^{2}+O\left(q^{3}\right) ; \\
\xi_{0,9}^{(2)} & =\phi_{0,1}\left(\phi_{0,4}\right)^{2}-5 \phi_{0,2} \phi_{0,3} \phi_{0,4}+4\left(\phi_{0,3}\right)^{3}=\left(r^{2}[-4]-r[-1]+4-\cdots\right) \\
& +\left(3 r^{6}-8 r^{5}+9 r^{4}-24 r^{3}+31 r^{2}-32 r+42-\cdots\right) q+\left(r^{9}[-9]+7 r^{8}-15 r^{7}\right. \\
& \left.+33 r^{6}-80 r^{5}+110 r^{4}-177 r^{3}+219 r^{2}-241 r+286-\cdots\right) q^{2}+O\left(q^{3}\right) ; \\
\xi_{0,9}^{(3)} & =E_{4,2} E_{4,3}\left(E_{4,1} \phi_{0,3}-E_{4} \phi_{0,4}\right) / \Delta_{12}-3 \phi_{0,1} \phi_{0,2}\left(\phi_{0,3}\right)^{2}+2\left(\phi_{0,1}\right)^{2} \phi_{0,3} \phi_{0,4} \\
& -30 \phi_{0,1}\left(\phi_{0,4}\right)^{2}+27 \phi_{0,2} \phi_{0,3} \phi_{0,4}+9\left(\phi_{0,3}\right)^{3}=q^{-1}[-36]+24 \\
& +\left(33 r^{6}+486 r^{5}+3159 r^{4}+10758 r^{3}+24057 r^{2}+37908 r+44082+\cdots\right) q \\
& +\left(2 r^{9}[-9]+243 r^{8}+5346 r^{7}+44055 r^{6}+204120 r^{5}+642978 r^{4}+1483416 r^{3}\right. \\
& \left.+2632905 r^{2}+3679020 r+4109590+\cdots\right) q^{2}+O\left(q^{3}\right) .
\end{aligned}
$$

We have $\bar{R}=\overline{P\left(\mathcal{M}_{0}\right)}$ where

$$
\begin{gathered}
P\left(\mathcal{M}_{0}\right)=\left(\begin{array}{ccc}
1 & 2 & 0 \\
0 & -1 & 0 \\
-1 & 0 & 1 \\
2 & 9 & 1
\end{array}\right) \equiv\left[\begin{array}{cc}
4 & \overline{2} \\
1 & \overline{1} \\
36 & \overline{0} \\
9 & \overline{9}
\end{array}\right] ; \operatorname{Mul}\left(P\left(\mathcal{M}_{0}\right), \xi\right)=\left(\begin{array}{ccc}
0 & 1 & 0 \\
3 & 0 & 0 \\
0 & 0 & 1 \\
-1 & 1 & 3
\end{array}\right) ; \\
G\left(P\left(\mathcal{M}_{0}\right)\right)=\left(\begin{array}{cccc}
4 & -2 & -18 & 0 \\
-2 & 1 & 0 & -9 \\
-18 & 0 & 36 & -18 \\
0 & -9 & -18 & 9
\end{array}\right) .
\end{gathered}
$$

Case $t=12$. The space $R J_{12}$ has the basis

$$
\begin{aligned}
\xi_{0,12}^{(1)} & =(\vartheta(\tau, z) / \eta(\tau))^{12}=\left(r^{8}[-16]-8 r^{7}[-1]+24 r^{6}-24 r^{5}-36 r^{4}+120 r^{3}\right. \\
& \left.-88 r^{2}-88 r+198-\cdots\right) q+\left(-4 r^{10}[-4]+24 r^{9}-32 r^{8}-104 r^{7}+396 r^{6}\right. \\
& \left.-352 r^{5}-512 r^{4}+1440 r^{3}-904 r^{2}-1008 r+2112-\cdots\right) q^{2}+O\left(q^{3}\right) ; \\
\xi_{0,12}^{(2)}= & 3 \phi_{0,2}\left(\phi_{0,3}\right)^{2} \phi_{0,4}-\left(\phi_{0,2}\right)^{2}\left(\phi_{0,4}\right)^{2}-2\left(\phi_{0,3}\right)^{4}-\left(\phi_{0,4}\right)^{3} \\
= & \left(r[-1]-1+r^{-1}[-1]\right)+\left(-r^{7}[-1]+r^{6}-r^{5}+r^{4}-r^{2}+2 r-2+\cdots\right) q
\end{aligned}
$$




$$
\begin{aligned}
\xi_{0,12}^{(3)} & =2\left(\phi_{0,2}\right)^{2}\left(\phi_{0,4}\right)^{2}-5 \phi_{0,2}\left(\phi_{0,3}\right)^{2} \phi_{0,4}+3\left(\phi_{0,3}\right)^{4}+\left(\phi_{0,4}\right)^{3}=\left(r^{2}[-4]-r[-1]\right. \\
& +3-\cdots)+\left(r^{7}[-1]-3 r^{6}+r^{5}-3 r^{4}+3 r^{3}-2 r+6-\cdots\right) q \\
& +\left(2 r^{10}[-4]-3 r^{8}+2 r^{7}-9 r^{6}+3 r^{5}-6 r^{4}+7 r^{2}-5 r+18-\cdots\right) q^{2}+O\left(q^{3}\right) ; \\
\xi_{0,12}^{(4)} & =E_{4,3}\left(E_{4,1} E_{4,2}\left(\phi_{0,3}\right)^{2}-2 E_{4} E_{4,2} \phi_{0,3} \phi_{0,4}+E_{4} E_{4,1}\left(\phi_{0,4}\right)^{2}\right) / \Delta_{12} \\
& -2\left(\phi_{0,1}\right)^{2} \phi_{0,2}\left(\phi_{0,4}\right)^{2}++5\left(\phi_{0,1}\right)^{2}\left(\phi_{0,3}\right)^{2} \phi_{0,4}-3 \phi_{0,1} \phi_{0,2}\left(\phi_{0,3}\right)^{3} \\
& -36 \phi_{0,1} \phi_{0,3}\left(\phi_{0,4}\right)^{2}+24 \phi_{0,2}\left(\phi_{0,3}\right)^{2} \phi_{0,4}+9\left(\phi_{0,3}\right)^{4}+16\left(\phi_{0,4}\right)^{3} \\
& =q^{-1}[-48]+24+\left(24 r^{7}[-1]+264 r^{6}+1608 r^{5}+5610 r^{4}+13464 r^{3}\right. \\
& \left.+24312 r^{2}+34056 r+38208+\cdots\right) q+\left(12 r^{10}[-4]+440 r^{9}+5544 r^{8}\right. \\
& +34104 r^{7}+135388 r^{6}+395808 r^{5}+902352 r^{4}+1667360 r^{3}+2550552 r^{2} \\
& +3276240 r+3558160+\cdots) q^{2}+O\left(q^{3}\right) .
\end{aligned}
$$

We have

$$
P\left(\mathcal{M}_{0}\right)=\left(\begin{array}{ccc}
1 & 2 & 0 \\
0 & -1 & 0 \\
-1 & 0 & 1 \\
1 & 8 & 1
\end{array}\right) \equiv\left[\begin{array}{cc}
4 & \overline{2} \\
1 & \overline{1} \\
48 & \overline{0} \\
16 & \overline{8}
\end{array}\right] ; G\left(P\left(\mathcal{M}_{0}\right)\right)=\left(\begin{array}{cccc}
4 & -2 & -24 & -8 \\
-2 & 1 & 0 & -8 \\
-24 & 0 & 48 & 0 \\
-8 & -8 & 0 & 16
\end{array}\right)
$$

$$
\bar{R}=\left[\begin{array}{c}
4, \overline{2} \\
1, \overline{1} \\
48, \overline{0} \\
16, \overline{8} \\
4, \overline{10} \\
1, \overline{7}
\end{array}\right] ; \quad \operatorname{Mul}(\bar{R}, \xi)=\left(\begin{array}{cccc}
0 & 0 & 1 & 0 \\
0 & 1 & 0 & 0 \\
0 & 0 & 0 & 1 \\
1 & 0 & 0 & 0 \\
-4 & -1 & 2 & 12 \\
-12 & -2 & 3 & 36
\end{array}\right)
$$

Case $t=16$. The space $R J_{16}$ has the basis

$$
\xi_{0,16}^{(1)}=\phi_{0,4}(\tau, 2 z)=\left(r^{2}[-4]+1+r^{-2}[-4]\right)+\left(-r^{8}-r^{6}+r^{2}+2+\cdots\right) q^{2}
$$




$$
\begin{aligned}
\xi_{0,16}^{(2)} & =E_{4,3}\left(E_{4} E_{4,1}\left(\phi_{0,3}\right)^{4}-\left(E_{4}\right)^{2}\left(\phi_{0,3}\right)^{3} \phi_{0,4}-2 E_{4,1} E_{4,2}\left(\phi_{0,3}\right)^{2} \phi_{0,4}\right. \\
& +E_{4} E_{4,2} \phi_{0,3}\left(\phi_{0,4}\right)^{2}-E_{4,1} E_{4,2}\left(\phi_{0,3}\right)^{2} \phi_{0,4}+2 E_{4} E_{4,2} \phi_{0,3}\left(\phi_{0,4}\right)^{2} \\
& \left.-E_{4} E_{4,1}\left(\phi_{0,4}\right)^{3}\right) / \Delta_{12}+2\left(\phi_{0,1}\right)^{3}\left(\phi_{0,3}\right)^{3} \phi_{0,4}-3\left(\phi_{0,1}\right)^{2} \phi_{0,2}\left(\phi_{0,3}\right)^{4} \\
& -7\left(\phi_{0,1}\right)^{2}\left(\phi_{0,3}\right)^{2}\left(\phi_{0,4}\right)^{2}-31 \phi_{0,1} \phi_{0,2}\left(\phi_{0,3}\right)^{3} \phi_{0,4}+46 \phi_{0,1}\left(\phi_{0,3}\right)^{5} \\
& +72 \phi_{0,1} \phi_{0,3}\left(\phi_{0,4}\right)^{3}+7\left(\phi_{0,2}\right)^{3}\left(\phi_{0,3}\right)^{2} \phi_{0,4}-72 \phi_{0,2}\left(\phi_{0,3}\right)^{2}\left(\phi_{0,4}\right)^{2} \\
& -197\left(\phi_{0,3}\right)^{4} \phi_{0,4}+2\left(\phi_{0,1}\right)^{2} \phi_{0,2}\left(\phi_{0,4}\right)^{3}+21\left(\phi_{0,3}\right)^{4} \phi_{0,4}-26\left(\phi_{0,4}\right)^{4} \\
& +2 \phi_{0,1}\left(\phi_{0,2}\right)^{2} \phi_{0,3}\left(\phi_{0,4}\right)^{2}-\left(\phi_{0,2}\right)^{2}\left(\phi_{0,3}\right)^{4}-4\left(\phi_{0,2}\right)^{2}\left(\phi_{0,4}\right)^{3}-2\left(\phi_{0,2}\right)^{4}\left(\phi_{0,4}\right)^{2} \\
& =q^{-1}[-64]+\left(8 r[-1]+14+8 r^{-1}[-1]\right)+\left(21 r^{8}+200 r^{7}+1036 r^{6}\right. \\
& \left.+3360 r^{5}+8100 r^{4}+15240 r^{3}+23604 r^{2}+30352 r+33058+\cdots\right) q \\
& +\left(56 r^{11}+1008 r^{10}+7336 r^{9}+32932 r^{8}+108800 r^{7}+283504 r^{6}+610344 r^{5}\right. \\
& \left.+1112832 r^{4}+1750728 r^{3}+2401952 r^{2}+2896688 r^{1}+3081400+\cdots\right) q^{2} \\
& +\left(4 r^{14}[-4]+560 r^{13}+8092 r^{12}+58328 r^{11}+283784 r^{10}+1042328 r^{9}\right. \\
& +3082176 r^{8}+7616904 r^{7}+16136000 r^{6}+29802144 r^{5}+48582612 r^{4} \\
& \left.+70497736 r^{3}+91619124 r^{2}+107054192 r+112732002+\cdots\right) q^{3}+O\left(q^{4}\right) .
\end{aligned}
$$

We have $\bar{R}=\overline{P\left(\mathcal{M}_{0}\right)}$ where

$$
\begin{gathered}
P\left(\mathcal{M}_{0}\right)=\left(\begin{array}{ccc}
1 & 2 & 0 \\
0 & -1 & 0 \\
-1 & 0 & 1 \\
5 & 32 & 3 \\
3 & 14 & 1
\end{array}\right) \equiv\left[\begin{array}{cc}
4 & \overline{2} \\
1 & \overline{1} \\
64 & \overline{0} \\
64 & \overline{0} \\
4 & \overline{14}
\end{array}\right] ; \operatorname{Mul}\left(P\left(\mathcal{M}_{0}\right), \xi\right)=\left(\begin{array}{ll}
1 & 0 \\
1 & 8 \\
0 & 1 \\
0 & 1 \\
1 & 4
\end{array}\right) ; \\
G\left(P\left(\mathcal{M}_{0}\right)\right)=\left(\begin{array}{ccccc}
4 & -2 & -32 & -32 & -4 \\
-2 & 1 & 0 & -32 & -14 \\
-32 & 0 & 64 & -64 & -64 \\
-32 & -32 & -64 & 64 & 0 \\
-4 & -14 & -64 & 0 & 4
\end{array}\right)
\end{gathered}
$$

Case $t=36$. The space $R J_{36}$ has the basis

$$
\begin{aligned}
\xi_{0,36}^{(1)} & =3 \xi_{0,36}^{(2)}-\xi_{0,9}^{(1)}(\tau, 2 z) \\
& =\left(-3 r[-1]+5-3 r^{-1}[-1]\right)+\left(r^{12}+3 r^{11}+\cdots\right) q \\
& +\left(r^{18}[-36]-3 r^{17}[-1]+9 r^{16}+\cdots\right) q^{2}+\left(6 r^{20}-3 r^{19}+\cdots\right) q^{3} \\
& +\left(4 r^{24}-15 r^{22}+\cdots\right) q^{4}+\left(3 r^{27}[-9]-9 r^{26}+3 r^{25}+\cdots\right) q^{5} \\
& +\left(3 r^{29}+6 r^{28}+\cdots\right) q^{6}+\left(3 r^{32}[-16]-25 r^{30}+9 r^{29}+\cdots\right) q^{7}
\end{aligned}
$$




$$
\begin{aligned}
& \xi_{0,36}^{(2)}=(\vartheta(\tau, 10 z) \vartheta(\tau, z)) /(\vartheta(\tau, 5 z) \vartheta(\tau, 2 z)) \\
& =\left(\left(r^{2}[-4]-r[-1]+1-r^{-1}[-1]+r^{-2}[-4]\right)+\left(-r^{12}+r^{11}-r^{10}+\cdots\right) q\right. \\
& +\left(-r^{17}[-1]+r^{16}-r^{15}+\cdots\right) q^{2}+\left(-r^{19}+2 r^{18}-3 r^{17}+\cdots\right) q^{3} \\
& +\left(-r^{21}+2 r^{20}-4 r^{19}+\cdots\right) q^{4}+\left(r^{27}[-9]-r^{26}+r^{25}+\cdots\right) q^{5} \\
& +\left(r^{29}-2 r^{28}+3 r^{27}+\cdots\right) q^{6}+\left(r^{32}[-16]-r^{30}+3 r^{29}+\cdots\right) q^{7} \\
& +\left(r^{34}[-4]-r^{33}+r^{32}-3 r^{30}+\cdots\right) q^{8}+O\left(q^{9}\right) \text {; } \\
& \xi_{0,36}^{(3)}=\left[\left(-36 \phi_{0,3}^{4}+56 \phi_{0,4}^{3}\right) \phi_{0,4}^{3} \phi_{0,3}^{3} E_{4}^{2}+\left(45 \phi_{0,3}^{8}-126 \phi_{0,4}^{3} \phi_{0,3}^{4}+\phi_{0,4}^{6}\right) \phi_{0,4}^{2} E_{4} E_{4,1}\right. \\
& +\left(-10 \phi_{0,3}^{8}+126 \phi_{0,4}^{3} \phi_{0,3}^{4}-8 \phi_{0,4}^{6}\right) \phi_{0,4} \phi_{0,3} E_{4} E_{4,2} \\
& \left.+\left(\phi_{0,3}^{8}-84 \phi_{0,4}^{3} \phi_{0,3}^{4}+28 \phi_{0,4}^{6}\right) \phi_{0,3}^{2} E_{4,1} E_{4,2}\right] E_{4,3} / \Delta \\
& +\left[770 \phi_{0,4}^{6} \phi_{0,3}^{2}-731 \phi_{0,4}^{7} \phi_{0,2}-731 \phi_{0,4}^{6} \phi_{0,2}^{3}+2924 \phi_{0,4}^{5} \phi_{0,3}^{2} \phi_{0,2}^{2}\right. \\
& -3655 \phi_{0,4}^{4} \phi_{0,3}^{4} \phi_{0,2}-29 \phi_{0,4}^{4} \phi_{0,3}^{2} \phi_{0,2}^{4}+133 \phi_{0,4}^{3} \phi_{0,3}^{4} \phi_{0,2}^{3}++1472 \phi_{0,4}^{3} \phi_{0,3}^{6} \\
& \left.-225 \phi_{0,4}^{2} \phi_{0,3}^{6} \phi_{0,2}^{2}+167 \phi_{0,4} \phi_{0,3}^{8} \phi_{0,2}-46 \phi_{0,3}^{10}\right] D_{0,6} \\
& +\left(72 \phi_{0,3}^{4}-112 \phi_{0,4}^{3}\right) \phi_{0,4}^{3} \phi_{0,3}^{3} \phi_{0,1}^{3}+\left[-731 \phi_{0,4}^{6} \phi_{0,2}^{3}+1462 \phi_{0,4}^{5} \phi_{0,3}^{2} \phi_{0,2}^{2}\right. \\
& +\left(-126 \phi_{0,4} \phi_{0,3}^{8}+1039 \phi_{0,4}^{4} \phi_{0,3}^{4}-733 \phi_{0,4}^{7}\right) \phi_{0,2} \\
& \left.+29 \phi_{0,3}^{10}-1615 \phi_{0,4}^{3} \phi_{0,3}^{6}-714 \phi_{0,4}^{6} \phi_{0,3}^{2}\right] \phi_{0,4} \phi_{0,1}^{2} \\
& +\left[12425 \phi_{0,4}^{6} \phi_{0,3} \phi_{0,2}^{4}-50600 \phi_{0,4}^{5} \phi_{0,3}^{3} \phi_{0,2}^{3}\right. \\
& +\left(67608 \phi_{0,4}^{4} \phi_{0,3}^{5}+20633 \phi_{0,4}^{7} \phi_{0,3}\right) \phi_{0,2}^{2}-\left(3 \phi_{0,3}^{11}+37314 \phi_{0,4}^{3} \phi_{0,3}^{7}\right. \\
& \left.\left.+35785 \phi_{0,4}^{6} \phi_{0,3}^{3}\right) \phi_{0,2}+8144 \phi_{0,4}^{2} \phi_{0,3}^{9}+17917 \phi_{0,4}^{5} \phi_{0,3}^{5}+8005 \phi_{0,4}^{8} \phi_{0,3}\right] \phi_{0,1} \\
& -29 \phi_{0,4}^{5} \phi_{0,2}^{8}+162 \phi_{0,4}^{4} \phi_{0,3}^{2} \phi_{0,2}^{7}-\left(358 \phi_{0,3}^{4}+10464 \phi_{0,4}^{3}\right) \phi_{0,4}^{3} \phi_{0,2}^{6} \\
& +\left(392 \phi_{0,3}^{4}+45141 \phi_{0,4}^{3}\right) \phi_{0,4}^{2} \phi_{0,3}^{2} \phi_{0,2}^{5}-\left(213 \phi_{0,3}^{8}+66918 \phi_{0,4}^{3} \phi_{0,3}^{4}\right. \\
& \left.+30811 \phi_{0,4}^{6}\right) \phi_{0,4} \phi_{0,2}^{4}+\left(46 \phi_{0,3}^{8}+43947 \phi_{0,4}^{3} \phi_{0,3}^{4}+83053 \phi_{0,4}^{6}\right) \phi_{0,3}^{2} \phi_{0,2}^{3} \\
& -\left(14354 \phi_{0,3}^{8}+64611 \phi_{0,4}^{3} \phi_{0,3}^{4}+30093 \phi_{0,4}^{6}\right) \phi_{0,4}^{2} \phi_{0,2}^{2} \\
& +\left(3426 \phi_{0,3}^{8}-496 \phi_{0,4}^{3} \phi_{0,3}^{4}+37331 \phi_{0,4}^{6}\right) \phi_{0,4} \phi_{0,3}^{2} \phi_{0,2}-569 \phi_{0,3}^{12} \\
& +3899 \phi_{0,4}^{3} \phi_{0,3}^{8}-455 \phi_{0,4}^{6} \phi_{0,3}^{4}-9861 \phi_{0,4}^{9}-83 \xi_{0,36}^{(1)} \\
& =q^{-1}[-144]+24+\left(24 r^{12}+72 r^{11}+\cdots\right) q \\
& +\left(4 r^{18}[-36]+144 r^{16}+672 r^{15}+\cdots\right) q^{2}+\left(144 r^{20}+1008 r^{19}+\cdots\right) q^{3} \\
& +\left(24 r^{24}+288 r^{23}+\cdots\right) q^{4}+\left(8 r^{27}[-9]+216 r^{26}+3096 r^{25}+\cdots\right) q^{5} \\
& +\left(72 r^{29}+1584 r^{28}+15720 r^{27}+\cdots\right) q^{6}+\left(9 r^{32}[-16]+288 r^{31}\right. \\
& \left.+5304 r^{30}+\cdots\right) q^{7}+\left(672 r^{33}+12096 r^{32}+\cdots\right) q^{8}+O\left(q^{9}\right)
\end{aligned}
$$

where

$$
\begin{aligned}
D_{0,6} & =(\theta(\tau, z) / \eta(q))^{12}=-\phi_{0,1}^{2} \phi_{0,4}+9 \phi_{0,1} \phi_{0,2} \phi_{0,3}-8 \phi_{0,2}^{3}-27 \phi_{0,3}^{2} \\
& =q\left(r^{6}-12 r^{5}+66 r^{4}-220 r^{3}+495 r^{2}-792 r+924-\ldots\right)+q^{2}(\ldots)
\end{aligned}
$$


(see $[33])$. We have

$$
\begin{aligned}
& P\left(\mathcal{M}_{0}\right)=\left(\begin{array}{ccc}
1 & 2 & 0 \\
0 & -1 & 0 \\
-1 & 0 & 1 \\
2 & 18 & 1 \\
5 & 27 & 1 \\
7 & 32 & 1
\end{array}\right) ; \quad G\left(P\left(\mathcal{M}_{0}\right)\right)=\left(\begin{array}{cccccc}
4 & -2 & -72 & -36 & -18 & -8 \\
-2 & 1 & 0 & -18 & -27 & -32 \\
-72 & 0 & 144 & -72 & -288 & -432 \\
-36 & -18 & -72 & 36 & -18 & -72 \\
-18 & -27 & -288 & -18 & 9 & 0 \\
-8 & -32 & -432 & -72 & 0 & 16
\end{array}\right) ; \\
& \bar{R}=\left[\begin{array}{c}
1, \overline{1} \\
1, \overline{17} \\
4, \overline{2} \\
4, \overline{34} \\
9, \overline{27} \\
16, \overline{32} \\
36, \overline{18} \\
144, \overline{0}
\end{array}\right] ; \quad \operatorname{Mul}(\bar{R}, \xi)=\left(\begin{array}{ccc}
-3 & 0 & 0 \\
-3 & 0 & 0 \\
0 & 1 & 0 \\
0 & 1 & 0 \\
4 & 1 & 12 \\
3 & 1 & 9 \\
1 & 0 & 4 \\
0 & 0 & 1
\end{array}\right) .
\end{aligned}
$$

2.6. The list of algebras of Theorem 2.1.1. In Table 2 below we give the list of all Lorentzian Kac-Moody algebras from Theorem 2.1.1. For each algebra from the list, the infinite product part of its denominator identity is defined by the infinite product $B_{\xi}$ of Theorem 2.2 .1 for some Jacobi form $\xi$ from the space $R J_{t}$ which is described in Table 1 of Sect. 2.5 by its bases. This product is characterized by the property that its multiplicities are equal to 0 or 1 for any rational quadratic divisor which is orthogonal to a root of $L_{t}$. Since $B_{\xi}$ is reflective, it is the whole divisor of $B_{\xi}$. We denote the corresponding Lorentzian Kac-Moody algebra as $\mathfrak{g}(\xi)$ since it is defined by the Jacobi form $\xi$.

We also describe the Fourier expansion of the automorphic form $B_{\xi}$, which gives the infinite sum part of the denominator identity of the algebra $\mathfrak{g}(\xi)$. For some of these forms only rational expressions from known Fourier expansions are known. These results were obtained in [36], [37], [39], [41]. We should say that these calculations are very non-trivial because there does not exist a general method of finding these Fourier expansions. We don't discuss these calculations in this paper.

We describe the fundamental chamber $\mathcal{M}$ and the set $P(\mathcal{M})$ of orthogonal roots to $\mathcal{M}$ defining the Weyl group and the set of simple real roots of the algebra $\mathfrak{g}(\xi)$. We also give the subset $P(\mathcal{M})_{\overline{1}} \subset P(\mathcal{M})$ of odd roots. It means that the corresponding generators $e_{\alpha}, f_{\alpha}, \alpha \in P(\mathcal{M})_{\overline{1}}$, should be super (odd). If we don't mention the set $P(\mathcal{M})_{\overline{1}}$, it is empty. We also give the generalized Cartan matrix

$$
A=\left(\frac{2\left(\alpha_{i}, \alpha_{j}\right)}{\alpha_{i}^{2}}\right), \quad \alpha_{i}, \alpha_{j} \in P(\mathcal{M}),
$$

which is the main invariant of the algebra. Many of these matrices will be matrices of Theorem 1.5.6 or were considered in [41, Sect. 5.1]. Then we follow notations there. We also give the Weyl vector $\rho$.

All these polygons $\mathcal{M}$ are composed from the fundamental polyhedron $\mathcal{M}_{0}$ for $W\left(S_{t}\right)$ using some group of symmetries of the polyhedron $\mathcal{M}$. We use these symmetries to describe the sets $P(\mathcal{M})$ and $P(\mathcal{M})_{\overline{1}}$ using the set $P\left(\mathcal{M}_{0}\right)$. We numerate elements $\alpha_{1}, \ldots, \alpha_{k}$ of $P\left(\mathcal{M}_{0}\right)$ as they are given in Table 1 . We denote by $s_{\alpha}$ the reflection in the root $\alpha$. It is given by the formula

$$
\frac{2(x, \alpha)}{\alpha} \quad x \in S^{*}
$$


We denote by $\left[g_{1}, \ldots, g_{k}\right]$ the group generated by $g_{1}, \ldots, g_{k}$.

\section{Table 2. The list of all Lorentzian Kac-Moody algebras with the root lattice $S_{t}^{*}$, symmetry lattice $L_{t}^{*}$ and the symmetry group $\widehat{O}\left(L_{t}\right)$}

\section{Case $t=1$}

The Algebra $\mathfrak{g}\left(\xi_{0,1}^{(1)}\right)$. The fundamental chamber $\mathcal{M}=\left[s_{\alpha_{1}}, s_{\alpha_{3}}\right]\left(\mathcal{M}_{0}\right)$ is the right triangle with zero angles. We have

$$
P(\mathcal{M})=\left[s_{\alpha_{1}}, s_{\alpha_{3}}\right]\left(\alpha_{2}\right)=\{(0,-1,0),(1,1,0),(0,1,1)\}
$$

with the group of symmetries $\left[s_{\alpha_{1}}, s_{\alpha_{3}}\right]$ which is the dihedral group $D_{3}$ of order 6 (we use the same notation in general for the dihedral group $D_{n}$ ). The generalized Cartan matrix is

$$
A_{1, I I}=\left(\begin{array}{rrr}
2 & -2 & -2 \\
-2 & 2 & -2 \\
-2 & -2 & 2
\end{array}\right)
$$

The Weyl vector $\rho=\left(\frac{1}{2}, \frac{1}{2}, \frac{1}{2}\right)$. The automorphic form $B_{\xi_{0,1}^{(1)}}$ coincides with the classical automorphic form $\Delta_{5}$ of the weight 5 which is product (divided by 64) of ten even theta-constants of genus 2. This automorphic form also gives the discriminant of genus 2 algebraic curves. By Maass [55],

$$
\Delta_{5}=\sum_{\substack{n, l, m \equiv 1 \bmod 2 \\ n, m>0}} \sum_{d \mid(n, l, m)}(-1)^{\frac{l+d+2}{2}} d^{4} \tau_{9}\left(\frac{4 n m-l^{2}}{d^{2}}\right) q^{n / 2} r^{l / 2} s^{m / 2},
$$

where $\eta(\tau)^{9}=\sum_{n \geq 1} \tau_{9}(n) q^{n / 24}$ (see $\S 3,(3.4)$ about $\eta(\tau)$ ). It gives the infinite sum part of the denominator identity of the algebra $\mathfrak{g}\left(\xi_{0,1}^{(1)}\right)$. Thus, the denominator identity of the algebra $\mathfrak{g}\left(\xi_{0,1}^{(1)}\right)$ has the form

$$
\begin{aligned}
& \sum_{\substack{n, l, m \equiv 1 \bmod 2 \\
n, m>0}} \sum_{a \mid(n, l, m)}(-1)^{\frac{l+a+2}{2}} a^{4} \tau_{9}\left(\frac{4 n m-l^{2}}{a^{2}}\right) q^{n / 2} r^{l / 2} s^{m / 2} \\
& =(q r s)^{1 / 2} \prod_{\substack{n, l, m \in \mathbb{Z} \\
(n, l, m)>0}}\left(1-q^{n} r^{l} s^{m}\right)^{f_{1}(n m, l)}
\end{aligned}
$$

where

$$
\xi_{0,1}^{(1)}(\tau, z)=\phi_{0,1}(\tau, z)=\sum_{k, l \in \mathbb{Z}} f_{1}(k, l) q^{k} r^{l}
$$

is Fourier expansion of the Jacobi form $\xi_{0,1}^{(1)}=\phi_{0,1}$ from Table 1 , case $t=1$, and $\phi_{0,1}$ 
Denominator identities like (2.6.1) can be similarly written in all cases below (since Fourier expansions of the corresponding automorphic forms $\xi$ and $B_{\xi}$ are known). We leave this to a reader.

The Algebra $\mathfrak{g}\left(\xi_{0,1}^{(2)}\right)$. The chamber $\mathcal{M}=\mathcal{M}_{0}$ is a triangle with angles $0, \pi / 2$, $\pi / 3$. The set $P(\mathcal{M})=P\left(\mathcal{M}_{0}\right), P(\mathcal{M})_{\overline{1}}=\left\{\alpha_{2}\right\}$. The generalized Cartan matrix is

$$
A_{1, I, \overline{0}}=A_{1, I, \overline{1}}=\left(\begin{array}{ccc}
2 & -1 & -1 \\
-4 & 2 & 0 \\
-1 & 0 & 2
\end{array}\right) .
$$

The Weyl vector $\rho=\left(\frac{5}{2}, \frac{1}{2}, \frac{3}{2}\right)$. The automorphic form $B_{\xi_{0,1}^{(2)}}$ coincides with Igusa's [46] modular form $\Delta_{30}=\Delta_{35} / \Delta_{5}$ of the weight 30. See [39], [41]. Fourier expansion of $\Delta_{35}$ was found by Igusa in [46]. Another expression for Fourier expansion of $\Delta_{35}$ as Hecke product of $\Delta_{5}$ was found in [39]. Let

$$
\begin{aligned}
{\left[\Delta_{5}(z)\right]_{T(2)}=} & \prod_{a, b, c} \Delta_{5}\left(\frac{z_{1}+a}{2}, \frac{z_{2}+b}{2}, \frac{z_{3}+c}{2}\right) \\
& \times \prod_{a \bmod 2} \Delta_{5}\left(\frac{z_{1}+a}{2}, z_{2}, 2 z_{3}\right) \Delta_{5}\left(2 z_{1}, z_{2}, \frac{z_{3}+a}{2}\right) \\
& \times \Delta_{5}\left(2 z_{1}, 2 z_{2}, 2 z_{3}\right) \prod_{b \bmod 2} \Delta_{5}\left(2 z_{1},-z_{1}+z_{2}, \frac{z_{1}-2 z_{2}+z_{3}+b}{2}\right) .
\end{aligned}
$$

In [39], it was shown that

$$
\Delta_{35}(z)=\frac{\left[\Delta_{5}(z)\right]_{T(2)}}{\Delta_{5}(z)^{8}} .
$$

Thus, $\Delta_{30}(z)=\left[\Delta_{5}(z)\right]_{T(2)} / \Delta_{5}(z)^{9}$. This gives Fourier expansions of $\Delta_{35}$ and $\Delta_{30}$ as finite products and quotients of known Fourier expansions. See [39] and [41] for details.

The Algebra $\mathfrak{g}\left(\xi_{0,1}^{(1)}+\xi_{0,1}^{(2)}\right)$. The chamber $\mathcal{M}=\mathcal{M}_{0}$ (the same as for $\left.\mathfrak{g}\left(\xi_{0,1}^{(2)}\right)\right)$ and

$$
P(\mathcal{M})=\left\{\alpha_{1}, 2 \alpha_{2}, \alpha_{3}\right\}
$$

The generalized Cartan matrix is

$$
A_{1,0}=\left(\begin{array}{ccc}
2 & -2 & -1 \\
-2 & 2 & 0 \\
-1 & 0 & 2
\end{array}\right)
$$

The Weyl vector $\rho=(3,1,2)$. The automorphic form $B_{\xi_{0,1}^{(1)}+\xi_{0,1}^{(2)}}$ coincides with Igusa's [46] modular form $\Delta_{35}$ of the weight 35 which has been considered above.

\section{Case $t=2$}

The Algebra $\mathfrak{g}\left(\xi_{0,2}^{(1)}\right)$. The chamber $\mathcal{M}=\left[s_{\alpha_{1}}, s_{\alpha_{3}}\right]\left(\mathcal{M}_{0}\right)$ is the right quadrangle with zero angles; the set 
with the group of symmetries $\left[s_{\alpha_{1}}, s_{\alpha_{3}}\right]$ which is $D_{4}$. The generalized Cartan matrix is

$$
A_{2, I I}=\left(\begin{array}{rrrr}
2 & -2 & -6 & -2 \\
-2 & 2 & -2 & -6 \\
-6 & -2 & 2 & -2 \\
-2 & -6 & -2 & 2
\end{array}\right)
$$

The Weyl vector $\rho=\left(\frac{1}{4}, \frac{1}{2}, \frac{1}{4}\right)$. The automorphic form $B_{\xi_{0,2}^{(1)}}$ coincides with the automorphic form $\Delta_{2}$ of the weight 2 which was introduced in [36] and [41]. Its Fourier expansion is

$$
\Delta_{2}=\sum_{N \geq 1} \sum_{\substack{n, m>0, l \in \mathbb{Z} \\ n, m \equiv 1 \bmod 4 \\ 2 n m-l^{2}=N^{2}}} N\left(\frac{-4}{N l}\right) \sum_{a \mid(n, l, m)}\left(\frac{-4}{a}\right) q^{n / 4} r^{l / 2} s^{m / 4}
$$

Here $\left(\frac{m}{n}\right)$ is the Jacobi symbol (or the generalized Legendre symbol).

The Algebra $\mathfrak{g}\left(\xi_{0,2}^{(2)}\right)$. The chamber $\mathcal{M}=\left[s_{\alpha_{3}}\right]\left(\mathcal{M}_{0}\right)$ is a triangle with angles $0,0, \pi / 2$. The sets

$$
P(\mathcal{M})=\left\{\alpha_{1}, \alpha_{2}, s_{\alpha_{3}}\left(\alpha_{1}\right)=(0,2,1)\right\}, \quad P(\mathcal{M})_{\overline{1}}=\left\{\alpha_{2}\right\}
$$

with the group of symmetries $\left[s_{\alpha_{3}}\right]$ which is $D_{1}$. The generalized Cartan matrix is

$$
A_{2, I, \overline{0}}=\left(\begin{array}{ccc}
2 & -1 & 0 \\
-4 & 2 & -4 \\
0 & -1 & 2
\end{array}\right)
$$

The Weyl vector $\rho=\left(\frac{3}{4}, \frac{1}{2}, \frac{3}{4}\right)$. The automorphic form $B_{\xi_{0,2}^{(2)}}$ coincides with the automorphic form $\Delta_{9}=\Delta_{11} / \Delta_{2}$ of the weight 9 from [41]. There are two formulae for the Fourier expansion of $\Delta_{11}$. This automorphic form is the lifting of its first Fourier-Jacobi coefficient :

$$
\Delta_{11}(z)=\operatorname{Lift}\left(\eta\left(z_{1}\right)^{21} \vartheta\left(z_{1}, 2 z_{2}\right)\right)
$$

It gives us a simple exact formula for the Fourier coefficients of $\Delta_{11}$ in terms of the Fourier coefficients of the Jacobi form $\eta\left(z_{1}\right)^{21} \vartheta\left(z_{1}, 2 z_{2}\right)$. This formula is similar to the formula for $\Delta_{5}$ above (see [41, Example 1.15]).

The second expression for $\Delta_{11}$ is given by the multiplicative symmetrisation of $\Delta_{5}$ for $t=1$ above. Let

$$
\operatorname{Ms}_{2}\left(\Delta_{5}\right)\left(z_{1}, z_{2}, z_{3}\right)=\Delta_{5}\left(z_{1}, 2 z_{2}, 4 z_{3}\right) \Delta_{5}\left(z_{1}, z_{2}, z_{3}\right) \Delta_{5}\left(z_{1}, z_{2}, z_{3}+1\right) .
$$

By $[41,(3.10)]$,

$$
\Delta_{11}(z)=\frac{\mathrm{Ms}_{2}\left(\Delta_{5}\right)(z)}{\Delta_{2}(z)^{2}} .
$$

Thus, $\Delta_{9}=\mathrm{Ms}_{2}\left(\Delta_{5}\right)(z) / \Delta_{2}(z)^{3}$. It gives Fourier expansions of $\Delta_{9}$ as finite prod- 
The Algebra $\mathfrak{g}\left(\xi_{0,2}^{(1)}+\xi_{0,2}^{(2)}\right)$. The polygon $\mathcal{M}=\left[s_{\alpha_{3}}\right]\left(\mathcal{M}_{0}\right)$ is a triangle with angles $0,0, \pi / 2$ (the same as for $\left.\mathfrak{g}\left(\xi_{0,2}^{(2)}\right)\right)$; the set

$$
P(\mathcal{M})=\left\{\alpha_{1}, 2 \alpha_{2}, s_{\alpha_{3}}\left(\alpha_{1}\right)=(0,2,1)\right\}
$$

with the group of symmetries $\left[s_{\alpha_{3}}\right]$ which is $D_{1}$. The generalized Cartan matrix is

$$
A_{2,0}=\left(\begin{array}{ccc}
2 & -2 & 0 \\
-2 & 2 & -2 \\
0 & -2 & 2
\end{array}\right) \text {. }
$$

The Weyl vector $\rho=(1,1,1)$. The automorphic form $B_{\xi_{0,2}^{(1)}+\xi_{0,2}^{(2)}}$ coincides with the $\Delta_{11}$ of the weight 11 . We have discussed its Fourier expansion above. See [39], [41].

The Algebra $\mathfrak{g}\left(\xi_{0,2}^{(3)}\right)$. The chamber $\mathcal{M}=\left[s_{\alpha_{1}}, s_{\alpha_{2}}\right]\left(\mathcal{M}_{0}\right)$ is an infinite polygon with angles $\pi / 2$ and which is touching a horosphere with the center at $\mathbb{R}_{++} \rho$ where $\rho=(1,0,0)$ is the Weyl vector. The set

$$
P(\mathcal{M})=\left[s_{\alpha_{1}}, s_{\alpha_{2}}\right]\left(\alpha_{3}\right)
$$

with the group of symmetries $\left[s_{\alpha_{1}}, s_{\alpha_{2}}\right]$ which is $D_{\infty}$. The generalized Cartan matrix is the symmetric matrix

$$
A_{2, \overline{1}}=\left(\frac{\left(\alpha, \alpha^{\prime}\right)}{4}\right), \quad \alpha, \alpha^{\prime} \in P(\mathcal{M})
$$

The automorphic form $B_{\xi_{0,2}^{(3)}}$ is $\Psi_{12}^{(2)}$ of the weight 12 from [41].

It was shown in [41, Remark 4.4] that $\Psi_{12}^{(2)}$ can be obtained as restriction (possibly with some multiplicative constant) of the Borcherds automorphic form $\Phi$ from (1.3.7). The Borcherds automorphic form $\Phi$ is defined on Hermitian symmetric domain $\Omega(2 H \oplus \mathcal{L})$ where $\mathcal{L}$ is Leech lattice. One should restrict $\Phi$ on the subdomain $\Omega(2 H+\mathbb{Z} v)$ where $v \in \mathcal{L}$ is a primitive element with $v^{2}=2 t$ where $t=2$ for this case. Thus, we have

$$
\Psi_{12}^{(2)}=c \Phi \mid \Omega(2 H+\mathbb{Z} v)
$$

where $c$ is some constant. It gives some Fourier expansion of $\Psi_{12}^{(2)}$. The same construction is valid for automorphic forms $\Psi_{12}^{(t)}$ which we consider below when $t=3$ and $t=4$.

The Algebra $\mathfrak{g}\left(\xi_{0,2}^{(1)}+\xi_{0,2}^{(3)}\right)$. The polygon $\mathcal{M}=\left[s_{\alpha_{1}}\right]\left(\mathcal{M}_{0}\right)$ is a quadrangle with angles $\pi / 2, \pi / 2, \pi / 2,0$; the set

$$
P(\mathcal{M})=\left[s_{\alpha_{1}}\right]\left\{\alpha_{2}, \alpha_{3}\right\}=\left\{\alpha_{2}, \alpha_{3},(1,4,1),(1,1,0)\right\}
$$

with the group of symmetries $\left[s_{\alpha_{1}}\right]$ which is $D_{1}$. The generalized Cartan matrix is

$$
A_{2, I I, \overline{1}}=\left(\begin{array}{cccc}
2 & 0 & -8 & -2 \\
0 & 2 & 0 & -1 \\
-1 & 0 & 2 & 0
\end{array}\right)
$$


The Weyl vector $\rho=\left(\frac{5}{4}, \frac{1}{2}, \frac{1}{4}\right)$. The automorphic form $B_{\xi_{0,2}^{(1)}+\xi_{0,2}^{(3)}}$ coincides with the automorphic form $\Delta_{14}=\Delta_{2} \Psi_{12}^{(2)}$ of the weight 14. (We must correct the case $(2, I I, \overline{1})$ in $\left[41\right.$, page 264] in this way.) Fourier expansion of $\Delta_{14}$ is product of the Fourier expansions of $\Delta_{2}$ and $\Psi_{12}^{(2)}$.

The Algebra $\mathfrak{g}\left(\xi_{0,2}^{(2)}+\xi_{0,2}^{(3)}\right)$. The polygon $\mathcal{M}=\mathcal{M}_{0}$ is the triangle with angles $0, \pi / 2, \pi / 4$; the set

$$
P(\mathcal{M})=P\left(\mathcal{M}_{0}\right), \quad P(\mathcal{M})_{\overline{1}}=\left\{\alpha_{2}\right\}
$$

with the trivial group of symmetries and with the generalized Cartan matrix

$$
A_{2, I, \overline{1}}=\left(\begin{array}{ccc}
2 & -1 & -2 \\
-4 & 2 & 0 \\
-1 & 0 & 2
\end{array}\right) .
$$

The Weyl vector $\rho=\left(\frac{7}{4}, \frac{1}{2}, \frac{3}{4}\right)$. The automorphic form $B_{\xi_{0,2}^{(2)}+\xi_{0,2}^{(3)}}$ coincides with the automorphic form $\Delta_{9} \Psi_{12}^{(2)}$ of the weight 21. Its Fourier expansion is product of the Fourier expansions of $\Delta_{9}$ and $\Psi_{12}^{(2)}$.

The Algebra $\mathfrak{g}\left(\xi_{0,2}^{(1)}+\xi_{0,2}^{(2)}+\xi_{0,2}^{(3)}\right)$. The polygon $\mathcal{M}=\mathcal{M}_{0}$ is the triangle with angles $0, \pi / 2, \pi / 4$ (the same as for the $\left.\mathfrak{g}\left(\xi_{0,2}^{(2)}+\xi_{0,2}^{(3)}\right)\right)$; the set

$$
P(\mathcal{M})=\left\{\alpha_{1}, 2 \alpha_{2}, \alpha_{3}\right\}
$$

with the trivial group of symmetries and with the generalized Cartan matrix

$$
A_{2,0, \overline{1}}=\left(\begin{array}{ccc}
2 & -2 & -2 \\
-2 & 2 & 0 \\
-1 & 0 & 2
\end{array}\right)
$$

The Weyl vector $\rho=(2,1,1)$. The automorphic form $B_{\xi_{0,2}^{(1)}+\xi_{0,2}^{(2)}+\xi_{0,2}^{(3)}}$ coincides with the automorphic form $\Delta_{2} \Delta_{9} \Psi_{12}^{(2)}$ of the weight 23. Its Fourier expansion is given by products of the Fourier expansions of $\Delta_{2}, \Delta_{9}$ and $\Psi_{12}^{(2)}$. See [41].

\section{Case $t=3$}

The Algebra $\mathfrak{g}\left(\xi_{0,3}^{(1)}\right)$. The chamber $\mathcal{M}=\left[s_{\alpha_{1}}, s_{\alpha_{3}}\right]\left(\mathcal{M}_{0}\right)$ is the right hexagon with zero angles,

$P(\mathcal{M})=\left[s_{\alpha_{1}}, s_{\alpha_{3}}\right]\left(\alpha_{2}\right)=\{(0,-1,0),(1,1,0),(2,5,1),(2,7,2),(1,5,2),(0,1,1)\}$

with the group of symmetries $\left[s_{\alpha_{1}}, s_{\alpha_{3}}\right]$ which is $D_{6}$. The generalized Cartan matrix is

$$
A_{3, I I}=\left(\begin{array}{rrrrrc}
2 & -2 & -10 & -14 & -10 & -2 \\
-2 & 2 & -2 & -10 & -14 & -10 \\
-10 & -2 & 2 & -2 & -10 & -14 \\
-14 & -10 & -2 & 2 & -2 & -10 \\
-10 & -14 & -10 & -2 & 2 & -2
\end{array}\right)
$$


The Weyl vector $\rho=\left(\frac{1}{6}, \frac{1}{2}, \frac{1}{6}\right)$. The automorphic form $B_{\xi_{0,3}^{(1)}}$ coincides with the automorphic form $\Delta_{1}$ of the weight 1 introduced in [41]. Its Fourier expansion is

$$
\Delta_{1}=\sum_{M \geq 1} \sum_{\substack{n, m>0, l \in \mathbb{Z} \\ n, m \equiv 1 \bmod 6 \\ 4 n m-3 l^{2}=M^{2}}}\left(\frac{-4}{l}\right)\left(\frac{12}{M}\right) \sum_{a \mid(n, l, m)}\left(\frac{6}{a}\right) q^{n / 6} r^{l / 2} s^{m / 6} .
$$

See [39], [41].

The Algebra $\mathfrak{g}\left(\xi_{0,3}^{(2)}\right)$. The chamber $\mathcal{M}=\left[s_{\alpha_{3}}\right]\left(\mathcal{M}_{0}\right)$ is a triangle with angles $0,0, \pi / 3$. The sets

$$
P(\mathcal{M})=\left\{\alpha_{1}, \alpha_{2}, s_{\alpha_{3}}\left(\alpha_{1}\right)=(0,2,1)\right\}, \quad P(\mathcal{M})_{\overline{1}}=\left\{\alpha_{2}\right\}
$$

with the group of symmetries $\left[s_{\alpha_{3}}\right]$ which is $D_{1}$. The generalized Cartan matrix is

$$
A_{3, I, \overline{0}}=\left(\begin{array}{ccc}
2 & -1 & -1 \\
-4 & 2 & -4 \\
-1 & -1 & 2
\end{array}\right)
$$

The Weyl vector $\rho=\left(\frac{1}{2}, \frac{1}{2}, \frac{1}{2}\right)$. The automorphic form $B_{\xi_{0,3}^{(2)}}$ coincides with the automorphic form $D_{6}$ of the weight 6 introduced in [41]. The form $D_{6}$ is the lifting of its first Fourier-Jacobi coefficient $\eta\left(z_{1}\right)^{11} \vartheta_{3 / 2}\left(z_{1}, z_{2}\right)$ where $\vartheta_{3 / 2}(\tau, z)=$ $\eta(\tau) \vartheta(\tau, 2 z) / \vartheta(\tau, z)$. Thus there is an exact formula for the Fourier coefficients of $D_{6}$ in terms of the Fourier coefficients of this Jacobi form (see [41, Example 1.17]).

Fourier expansion of $\Delta_{1} D_{6}$ is also given by the finite Hecke product of $\Delta_{1}$ (which is similar to the Fourier expansion of $\Delta_{35}$ for $t=1$ above). Let

$$
\begin{aligned}
{\left[\Delta_{1}(z)\right]_{T(2)} } & =\prod_{a, b, c \bmod 2} \Delta_{1}\left(\frac{z_{1}+a}{2}, \frac{z_{2}+b}{2}, \frac{z_{3}+c}{2}\right) \\
& \times \prod_{a \bmod 2} \Delta_{1}\left(\frac{z_{1}+a}{2}, z_{2}, 2 z_{3}\right) \Delta_{1}\left(2 z_{1}, z_{2}, \frac{z_{3}+a}{2}\right) \\
& \times \Delta_{1}\left(2 z_{1}, 2 z_{2}, 2 z_{3}\right) \prod_{b \bmod 2} \Delta_{1}\left(2 z_{1},-z_{1}+z_{2}, \frac{z_{1}-2 z_{2}+z_{3}+b}{2}\right) .
\end{aligned}
$$

In [41], it is shown that

$$
\Delta_{1}(z) D_{6}(z)=\frac{2^{22}\left[\Delta_{1}(z)\right]_{T(2)}}{\Delta_{1}(z)^{8}} .
$$

Thus, Fourier expansion of $\Delta_{1} D_{6}$ is given by finite products and quotients of known Fourier expansions. See [39], [41] for details.

The Algebra $\mathfrak{g}\left(\xi_{0,3}^{(1)}+\xi_{0,3}^{(2)}\right)$. The polygon $\mathcal{M}=\left[s_{\alpha_{3}}\right]\left(\mathcal{M}_{0}\right)$ is a triangle with angles $0,0, \pi / 3$ (the same as for $\left.\xi_{0,3}^{(2)}\right)$; the set 
with the group of symmetries $\left[s_{\alpha_{3}}\right]$ which is $D_{2}$. The generalized Cartan matrix is

$$
A_{3,0}=\left(\begin{array}{ccc}
2 & -2 & -1 \\
-2 & 2 & -2 \\
-1 & -2 & 2
\end{array}\right) \text {. }
$$

The Weyl vector $\rho=\left(\frac{2}{3}, 1, \frac{2}{3}\right)$. The automorphic form $B_{\xi_{0,3}^{(1)}+\xi_{0,3}^{(2)}}$ is $\Delta_{1} D_{6}$ of the weight 7 . We have discussed Fourier expansion of $\Delta_{1} D_{6}$ above. See [39], [41].

The Algebra $\mathfrak{g}\left(\xi_{0,3}^{(3)}\right)$. The chamber $\mathcal{M}=\left[s_{\alpha_{1}}, s_{\alpha_{2}}\right]\left(\mathcal{M}_{0}\right)$ is an infinite polygon with angles $\pi / 3$ and which is touching a horosphere with the center at the Weyl vector $\rho=(1,0,0)$. The set

$$
P(\mathcal{M})=\left[s_{\alpha_{1}}, s_{\alpha_{2}}\right]\left(\alpha_{3}\right)
$$

with the group of symmetries $\left[s_{\alpha_{1}}, s_{\alpha_{2}}\right]$ which is $D_{\infty}$. The generalized Cartan matrix is the symmetric matrix

$$
A_{3, \overline{1}}=\left(\frac{\left(\alpha, \alpha^{\prime}\right)}{6}\right), \quad \alpha, \alpha^{\prime} \in P(\mathcal{M}) .
$$

The automorphic form $B_{\xi_{0,3}^{(3)}}$ coincides with the automorphic form $\Psi_{12}^{(3)}$ of the weight 12 from [41]. Like $\Psi_{12}^{(2)}$ above, the automorphic form $\Psi_{12}^{(3)}$ and its Fourier expansion can be obtained as the restriction of Borcherds automorphic form $\Phi$ from (1.3.7). See [41, Remark 4.4].

The Algebra $\mathfrak{g}\left(\xi_{0,3}^{(1)}+\xi_{0,3}^{(3)}\right)$. The polygon $\mathcal{M}=\left[s_{\alpha_{1}}\right]\left(\mathcal{M}_{0}\right)$ is a quadrangle with angles $0, \pi / 2, \pi / 3, \pi / 2$; the set

$$
P(\mathcal{M})=\left[s_{\alpha_{1}}\right]\left\{\alpha_{2}, \alpha_{3}\right\}=\left\{\alpha_{2}, \alpha_{3},(2,6,1),(1,1,0)\right\}
$$

with the group of symmetries $\left[s_{\alpha_{1}}\right]$ which is $D_{1}$. The generalized Cartan matrix is

$$
A_{3, I I, \overline{1}}=\left(\begin{array}{cccc}
2 & 0 & -12 & -2 \\
0 & 2 & -1 & -1 \\
-1 & -1 & 2 & 0 \\
-2 & -12 & 0 & 2
\end{array}\right)
$$

The Weyl vector $\rho=\left(\frac{7}{6}, \frac{1}{2}, \frac{1}{6}\right)$. The automorphic form $B_{\xi_{0,3}^{(1)}+\xi_{0,3}^{(3)}}$ coincides with $\Delta_{1} \Psi_{12}^{(3)}$ of the weight 13 . (We must correct the case $(3, I I, \overline{1})$ in $[41$, page 264$]$ in this way.) Its Fourier expansion is product of the Fourier expansions of $\Delta_{1}$ and $\Psi_{12}^{(3)}$.

The Algebra $\mathfrak{g}\left(\xi_{0,3}^{(2)}+\xi_{0,3}^{(3)}\right)$. The polygon $\mathcal{M}=\mathcal{M}_{0}$ is the triangle with angles $0, \pi / 2, \pi / 6$; the set

$$
P(\mathcal{M})=P\left(\mathcal{M}_{0}\right), \quad P(\mathcal{M})_{\overline{1}}=\left\{\alpha_{2}\right\}
$$

with the trivial group of symmetries and with the generalized Cartan matrix

$$
A_{3, I, \overline{1}}=\left(\begin{array}{ccc}
2 & -1 & -3 \\
-4 & 2 & 0 \\
1 & 0
\end{array}\right)
$$


The Weyl vector $\rho=\left(\frac{3}{2}, \frac{1}{2}, \frac{1}{2}\right)$. The automorphic form $B_{\xi_{0,3}^{(2)}+\xi_{0,3}^{(3)}}$ coincides with $D_{6} \Psi_{12}^{(3)}$ of the weight 18. See [41]. Its Fourier expansion is product of the Fourier expansions of $D_{6}$ and $\Psi_{12}^{(3)}$.

The Algebra $\mathfrak{g}\left(\xi_{0,3}^{(1)}+\xi_{0,3}^{(2)}+\xi_{0,3}^{(3)}\right)$. The polygon $\mathcal{M}=\mathcal{M}_{0}$ is the triangle with angles $0, \pi / 2, \pi / 6$ (the same as for the $\left.\mathfrak{g}\left(\xi_{0,3}^{(2)}+\xi_{0,3}^{(3)}\right)\right)$; the set

$$
P(\mathcal{M})=\left\{\alpha_{1}, 2 \alpha_{2}, \alpha_{3}\right\}
$$

with the trivial group of symmetries and with the generalized Cartan matrix

$$
A_{3,0, \overline{1}}=\left(\begin{array}{ccc}
2 & -2 & -3 \\
-2 & 2 & 0 \\
-1 & 0 & 2
\end{array}\right)
$$

The Weyl vector $\rho=\left(\frac{5}{3}, 1, \frac{2}{3}\right)$. The automorphic form $B_{\xi_{0,3}^{(1)}+\xi_{0,3}^{(2)}+\xi_{0,3}^{(3)}}$ is $\Delta_{1} D_{6} \Psi_{12}^{(3)}$ of the weight 19. See [41]. Its Fourier expansion is product of the Fourier expansions of $\Delta_{1}, D_{6}$ and $\Psi_{12}^{(3)}$.

\section{Case $t=4$}

The Algebra $\mathfrak{g}\left(\xi_{0,4}^{(1)}\right)$. The chamber $\mathcal{M}=\left[s_{\alpha_{1}}, s_{\alpha_{3}}\right]\left(\mathcal{M}_{0}\right)$ is the infinite polygon with zero angles touching a horosphere with the center at $\mathbb{R}_{++} \rho$, where $\rho=\left(\frac{1}{8}, \frac{1}{2}, \frac{1}{8}\right)$ is the Weyl vector; the set

$$
P(\mathcal{M})=\left[s_{\alpha_{1}}, s_{\alpha_{3}}\right]\left(\alpha_{2}\right)
$$

with the group of symmetries $\left[s_{\alpha_{1}}, s_{\alpha_{3}}\right]$ which is $D_{\infty}$. The generalized Cartan matrix is

$$
A_{4, I I, \overline{0}}=\left(2\left(\alpha, \alpha^{\prime}\right)\right), \quad \alpha, \alpha^{\prime} \in P(\mathcal{M})
$$

The automorphic form $B_{\xi_{0,4}^{(1)}}$ coincides with $\Delta_{1 / 2}$ of the weight $1 / 2$ which is the theta-constant of the genus 2. Its Fourier expansion is

$$
\Delta_{1 / 2}=\frac{1}{2} \sum_{n, m \in \mathbb{Z}}\left(\frac{-4}{n}\right)\left(\frac{-4}{m}\right) q^{n^{2} / 8} r^{n m / 2} s^{m^{2} / 8} .
$$

See [41].

The Algebra $\mathfrak{g}\left(\xi_{0,4}^{(2)}\right)$. The chamber $\mathcal{M}=\left[s_{\alpha_{3}}\right]\left(\mathcal{M}_{0}\right)$ is a triangle with angles $0,0,0$. The sets

$$
P(\mathcal{M})=\left\{\alpha_{1}, \alpha_{2}, s_{\alpha_{3}}\left(\alpha_{1}\right)=(0,2,1)\right\} ; \quad P(\mathcal{M})_{\overline{1}}=\left\{\alpha_{2}\right\}
$$

with the group of symmetries $\left[s_{\alpha_{3}}\right]$ which is $D_{1}$. The generalized Cartan matrix is

$$
A_{4, I, \overline{0}}=\left(\begin{array}{ccc}
2 & -1 & -2 \\
-4 & 2 & -4 \\
- & 1
\end{array}\right)
$$


The Weyl vector $\rho=\left(\frac{3}{8}, \frac{1}{2}, \frac{3}{8}\right)$. The automorphic form $B_{\xi_{0,4}^{(2)}}$ coincides with $\Delta_{5}^{(4)} / \Delta_{1 / 2}$ of the weight $\frac{9}{2}$ where $\Delta_{5}^{(4)}=\Delta_{5}\left(z_{1}, 2 z_{2}, z_{3}\right)$ and $\Delta_{5}(z)$ was used for $t=1$. Thus, $\Delta_{5}^{(4)}$ has Fourier expansion

$$
\Delta_{5}^{(4)}=\sum_{\substack{n, l, m \equiv 1 \bmod 2 \\ n, m>0}} \sum_{d \mid(n, l, m)}(-1)^{\frac{l+d+2}{2}} d^{4} \tau_{9}\left(\frac{4 n m-l^{2}}{d^{2}}\right) q^{n / 2} r s^{m / 2}
$$

and Fourier expansion of $\Delta_{5}^{(4)} / \Delta_{1 / 2}$ is quotient of the Fourier expansions of $\Delta_{5}^{(4)}$ and $\Delta_{1 / 2}$. See [41].

The Algebra $\mathfrak{g}\left(\xi_{0,4}^{(1)}+\xi_{0,4}^{(2)}\right)$. The polygon $\mathcal{M}=\left[s_{\alpha_{3}}\right]\left(\mathcal{M}_{0}\right)$ is a triangle with angles $0,0,0$ (the same as for $\mathfrak{g}\left(\xi_{0,1}^{(1)}\right)$ and $\left.\mathfrak{g}\left(\xi_{0,4}^{(2)}\right)\right)$; the set

$$
P(\mathcal{M})=\left\{\alpha_{1}, 2 \alpha_{2}, s_{\alpha_{3}}\left(\alpha_{1}\right)=(0,2,1)\right\}
$$

with the group of symmetries $\left[s_{\alpha_{3}}\right]$ which is $D_{1}$. The generalized Cartan matrix is

$$
A_{4,0, \overline{0}}=A_{1, I I}=\left(\begin{array}{ccc}
2 & -2 & -2 \\
-2 & 2 & -2 \\
-2 & -2 & 2
\end{array}\right)
$$

(it is the same as for $\left.\mathfrak{g}\left(\xi_{0,1}^{(1)}\right)\right)$. The Weyl vector $\rho=\left(\frac{1}{2}, 1, \frac{1}{2}\right)$. The automorphic form $B_{\xi_{0,4}^{(1)}+\xi_{0,4}^{(2)}}$ coincides with $\Delta_{5}^{(4)}\left(z_{1}, z_{2}, z_{3}\right)=\Delta_{5}\left(z_{1}, 2 z_{2}, z_{3}\right)$ of the weight 5 with Fourier expansion above. This case is equivalent to the case $\mathfrak{g}\left(\xi_{0,1}^{(1)}\right)$ above. See [41].

The Algebra $\mathfrak{g}\left(\xi_{0,4}^{(3)}\right)$. The chamber $\mathcal{M}=\left[s_{\alpha_{1}}, s_{\alpha_{2}}\right]\left(\mathcal{M}_{0}\right)$ is an infinite polygon with zero angles which is touching a horosphere with the center at $\mathbb{R}_{++} \rho$, where $\rho=(1,0,0)$ is the Weyl vector. The set

$$
P(\mathcal{M})=\left[s_{\alpha_{1}}, s_{\alpha_{2}}\right]\left(\alpha_{3}\right)
$$

with the group of symmetries $\left[s_{\alpha_{1}}, s_{\alpha_{2}}\right]$ which is $D_{\infty}$. The generalized Cartan matrix is the symmetric matrix

$$
A_{4, \overline{1}}=\left(\frac{\left(\alpha, \alpha^{\prime}\right)}{8}\right), \quad \alpha, \alpha^{\prime} \in P(\mathcal{M})
$$

The automorphic form $B_{\xi_{0,4}^{(3)}}$ coincides with the automorphic form $\Psi_{12}^{(4)}$ of the weight 12 from [41]. Like $\Psi_{12}^{(2)}$ above, the automorphic form $\Psi_{12}^{(4)}$ and its Fourier expansion can be obtained as restriction of the Borcherds automorphic form $\Phi$ from (1.3.7). See [41, Remark 4.4].

The Algebra $\mathfrak{g}\left(\xi_{0,4}^{(1)}+\xi_{0,4}^{(3)}\right)$. The polygon $\mathcal{M}=\left[s_{\alpha_{1}}\right]\left(\mathcal{M}_{0}\right)$ is a quadrangle with angles $\pi / 2,0, \pi / 2,0$; the set 
with the group of symmetries $\left[s_{\alpha_{1}}\right]$ which is $D_{1}$. The generalized Cartan matrix is

$$
A_{4, I I, \overline{1}}=\left(\begin{array}{cccc}
2 & 0 & -16 & -2 \\
0 & 2 & -2 & -1 \\
-1 & -2 & 2 & 0 \\
-2 & -16 & 0 & 2
\end{array}\right)
$$

The Weyl vector $\rho=\left(\frac{9}{8}, \frac{1}{2}, \frac{1}{8}\right)$. The automorphic form $B_{\xi_{0,4}^{(1)}+\xi_{0,4}^{(3)}}$ coincides with $\Delta_{1 / 2} \Psi_{12}^{(4)}$ of the weight $\frac{25}{2}$. (We must correct the case $(4, I I, \overline{1})$ in [41, page 264] in this way.) Its Fourier expansion is product of the Fourier expansions of $\Delta_{1 / 2}$ and $\Psi_{12}^{(4)}$.

The Algebra $\mathfrak{g}\left(\xi_{0,4}^{(2)}+\xi_{0,4}^{(3)}\right)$. The polygon $\mathcal{M}=\mathcal{M}_{0}$ is the triangle with angles $0, \pi / 2,0 ;$ the sets

$$
P(\mathcal{M})=P\left(\mathcal{M}_{0}\right), \quad P(\mathcal{M})_{\overline{1}}=\left\{\alpha_{2}\right\}
$$

with the trivial group of symmetries and with the generalized Cartan matrix

$$
A_{4, I, \overline{1}}=\left(\begin{array}{ccc}
2 & -1 & -4 \\
-4 & 2 & 0 \\
-1 & 0 & 2
\end{array}\right)
$$

The Weyl vector $\rho=\left(\frac{11}{8}, \frac{1}{2}, \frac{3}{8}\right)$. The automorphic form $B_{\xi_{0,4}^{(2)}+\xi_{0,4}^{(3)}}$ coincides with $\Psi_{12}^{(4)} \Delta_{5}^{(4)} / \Delta_{1 / 2}$ of the weight $\frac{33}{2}$. Its Fourier expansion is product and quotient of known Fourier expansions of $\Psi_{12}^{(4)}, \Delta_{5}^{(4)}$ and $\Delta_{1 / 2}$. See [41].

The Algebra $\mathfrak{g}\left(\xi_{0,4}^{(1)}+\xi_{0,4}^{(2)}+\xi_{0,4}^{(3)}\right)$. The polygon $\mathcal{M}=\mathcal{M}_{0}$ is the triangle with angles $0, \pi / 2,0$ (the same as for the $\left.\mathfrak{g}\left(\xi_{0,4}^{(2)}+\xi_{0,4}^{(3)}\right)\right)$; the set

$$
P(\mathcal{M})=\left\{\alpha_{1}, 2 \alpha_{2}, \alpha_{3}\right\}
$$

with the trivial group of symmetries and with the generalized Cartan matrix

$$
A_{4,0, \overline{1}}=\left(\begin{array}{ccc}
2 & -2 & -4 \\
-2 & 2 & 0 \\
-1 & 0 & 2
\end{array}\right)
$$

The Weyl vector $\rho=\left(\frac{3}{2}, 1, \frac{1}{2}\right)$. The automorphic form $B_{\xi_{0,4}^{(1)}+\xi_{0,4}^{(2)}+\xi_{0,4}^{(3)}}$ coincides with $\Delta_{5}^{(4)} \Psi_{12}^{(4)}$ of the weight 17 . Its Fourier expansion is product of the Fourier expansions of $\Delta_{5}^{(4)}$ and $\Psi_{12}^{(4)}$. See [41].

\section{Case $t=8$}

The Algebra $\mathfrak{g}\left(\xi_{0,8}^{(2)}\right)$. The chamber $\mathcal{M}=\left[s_{\alpha_{3}}\right]\left(\mathcal{M}_{0}\right)$ is the right quadrangle with zero angles; the set 
with the group of symmetries $\left[s_{\alpha_{3}}\right]$ which is $D_{1}$. The generalized Cartan matrix is $A_{2, I I}$ (the same as for $\mathfrak{g}\left(\xi_{0,2}^{(1)}\right)$ for $t=2$ ). The Weyl vector $\rho=\left(\frac{1}{4}, 1, \frac{1}{4}\right)$. The automorphic form $B_{\xi_{0,8}^{(2)}}$ coincides with $\Delta_{2}^{(8)}\left(z_{1}, z_{2}, z_{3}\right)=\Delta_{2}\left(z_{1}, 2 z_{2}, z_{3}\right)$ of the weight 2 where $\Delta_{2}$ corresponds to $\mathfrak{g}\left(\xi_{0,2}^{(1)}\right)$. Thus, Fourier expansion of $\Delta_{2}^{(8)}$ is

$$
\Delta_{2}^{(8)}=\sum_{N \geq 1} \sum_{\substack{n, m>0, l \in \mathbb{Z} \\ n, m \equiv 1 \text { mod } 4 \\ 2 n m-l^{2}=N^{2}}} N\left(\frac{-4}{N l}\right) \sum_{a \mid(n, l, m)}\left(\frac{-4}{a}\right) q^{n / 4} r s^{m / 4} .
$$

This case is equivalent to $\mathfrak{g}\left(\xi_{0,2}^{(1)}\right)$.

$$
\text { Case } t=9
$$

The algebra $\mathfrak{g}\left(\xi_{0,9}^{(2)}\right)$. The chamber $\mathcal{M}=\left[s_{\alpha_{3}}\right]\left(\mathcal{M}_{0}\right)$ is the pentagon with angles $0,0, \pi / 2,0, \pi / 2$; the set

$$
\begin{aligned}
& P(\mathcal{M})=\left[s_{\alpha_{3}}\right]\left(\alpha_{1}, \alpha_{2}, \alpha_{4}\right)=\left\{\alpha_{1}, \alpha_{2}, s_{\alpha_{3}}\left(\alpha_{1}\right)=(0,2,1), s_{\alpha_{3}}\left(\alpha_{4}\right)=(1,9,2), \alpha_{4}\right\} \\
& P(\mathcal{M})_{\overline{1}}=\left\{\alpha_{2}\right\}
\end{aligned}
$$

with the group of symmetries $\left[s_{\alpha_{3}}\right]$ which is $D_{1}$. The generalized Cartan matrix is

$$
\left(\begin{array}{ccccc}
2 & -1 & -7 & -9 & 0 \\
-4 & 2 & -4 & -18 & -18 \\
-7 & -1 & 2 & 0 & -9 \\
-4 & -2 & 0 & 2 & -2 \\
0 & -2 & -4 & -2 & 2
\end{array}\right) .
$$

The Weyl vector $\rho=\left(\frac{1}{6}, \frac{1}{2}, \frac{1}{6}\right)$. The automorphic form $B_{\xi_{0,9}^{(2)}}$ coincides with the automorphic form $D_{2}$ of the weight 2 with Fourier expansion

$$
D_{2}=\sum_{N \geq 1} \sum_{\substack{m>0, l \in \mathbb{Z} \\ n, m \equiv 1 \text { mod } 6 \\ 4 n m-l^{2}=N^{2}}} N\left(\frac{-4}{N}\right)\left(\frac{12}{l}\right) \sum_{a \mid(n, l, m)}\left(\frac{6}{a}\right) q^{n / 6} r^{l / 2} s^{m / 6} .
$$

See $[41,(5.1 .2)]$.

\section{Case $t=12$}

The Algebra $\mathfrak{g}\left(\xi_{0,12}^{(2)}+\xi_{0,12}^{(3)}\right)$. The chamber $\mathcal{M}=\left[s_{\alpha_{3}}, s_{\alpha_{4}}\right]\left(\mathcal{M}_{0}\right)$ is the right hexagon with zero angles; the set

$$
\begin{aligned}
P(\mathcal{M})= & {\left[s_{\alpha_{3}}, s_{\alpha_{4}}\right]\left(\alpha_{1}, 2 \alpha_{2}\right)=\left\{\alpha_{1}, 2 \alpha_{2}, s_{\alpha_{3}}\left(\alpha_{1}\right)=(0,2,1),\right.} \\
& \left.s_{\alpha_{4}} s_{\alpha_{3}}\left(\alpha_{1}\right)=(1,10,2), s_{\alpha_{4}}\left(2 \alpha_{2}\right)=(2,14,2), s_{\alpha_{4}}\left(\alpha_{1}\right)=(2,10,1)\right\}
\end{aligned}
$$

with the group of symmetries $\left[s_{\alpha_{3}}, s_{\alpha_{4}}\right]$ which is $D_{4}$. The generalized Cartan matrix 
form $B_{\xi_{0,12}^{(2)}+\xi_{0,12}^{(3)}}$ is $\Delta_{1}^{(12)}\left(z_{1}, z_{2}, z_{3}\right)=\Delta_{1}\left(z_{1}, 2 z_{2}, z_{3}\right)$ of the weight 1 where $\Delta_{1}$ corresponds to $\mathfrak{g}\left(\xi_{0,3}^{(1)}\right)$. Thus, Fourier expansion of $\Delta_{1}^{(12)}$ is

$$
\Delta_{1}^{(12)}=\sum_{\substack { M \geq 1 \\
\begin{subarray}{c}{n, m>0, l \in \mathbb{Z} \\
n, m \equiv 1 \bmod 6 \\
4 n m-3 l^{2}=M^{2}{ M \geq 1 \\
\begin{subarray} { c } { n , m > 0 , l \in \mathbb { Z } \\
n , m \equiv 1 \operatorname { m o d } 6 \\
4 n m - 3 l ^ { 2 } = M ^ { 2 } } }\end{subarray}}\left(\frac{-4}{l}\right)\left(\frac{12}{M}\right) \sum_{a \mid(n, l, m)}\left(\frac{6}{a}\right) q^{n / 6} r s^{m / 6} .
$$

This case is equivalent to $\mathfrak{g}\left(\xi_{0,3}^{(1)}\right)$ above.

\section{Case $t=16$}

The Algebra $\mathfrak{g}\left(\xi_{0,16}^{(1)}\right)$. The chamber $\mathcal{M}=\left[s_{\alpha_{3}}, s_{\alpha_{4}}\right]\left(\mathcal{M}_{0}\right)$ is an infinite polygon with zero angles touching a horosphere with the center $\mathbb{R}_{++} \rho$ where $\rho=\left(\frac{1}{8}, 1, \frac{1}{8}\right)$ is the Weyl vector. The set

$$
P(\mathcal{M})=\left[s_{\alpha_{3}}, s_{\alpha_{4}}\right]\left(\alpha_{1}, 2 \alpha_{2}, \alpha_{5}\right)
$$

with the group of symmetries $\left[s_{\alpha_{3}}, s_{\alpha_{4}}\right]$ which is $D_{\infty}$. The generalized Cartan matrix is

$$
\left(\frac{\left(\alpha, \alpha^{\prime}\right)}{2}\right), \quad \alpha, \alpha^{\prime} \in P(\mathcal{M})
$$

which is the same as for $\mathfrak{g}\left(\xi_{0,4}^{(1)}\right)$. The automorphic form $B_{\xi_{0,16}^{(1)}}$ coincides with $\Delta_{1 / 2}^{(16)}\left(z_{1}, z_{2}, z_{3}\right)=\Delta_{1 / 2}\left(z_{1}, 2 z_{2}, z_{3}\right)$ of the weight $1 / 2$ where $\Delta_{1 / 2}$ corresponds to $\mathfrak{g}\left(\xi_{0,4}^{(1)}\right)$. Thus, Fourier expansion of $\Delta_{1 / 2}^{(16)}$ is

$$
\Delta_{1 / 2}^{(16)}=\frac{1}{2} \sum_{n, m \in \mathbb{Z}}\left(\frac{-4}{n}\right)\left(\frac{-4}{m}\right) q^{n^{2} / 8} r^{n m} s^{m^{2} / 8} .
$$

This case is equivalent to $\mathfrak{g}\left(\xi_{0,4}^{(1)}\right)$.

\section{Case $t=36$}

The Algebra $\mathfrak{g}\left(\xi_{0,36}^{(2)}\right)$. The chamber $\mathcal{M}=\left[s_{\alpha_{3}}, s_{\alpha_{4}}\right]\left(\mathcal{M}_{0}\right)$ is the infinite periodic polygon with angles $\ldots, 0, \pi / 2,0,0,0,0, \pi / 2,0, \ldots$, with the center at the Weyl vector $\rho=\left(\frac{1}{24}, \frac{1}{2}, \frac{1}{24}\right)$ at infinity. The set

$$
P(\mathcal{M})=\left[s_{\alpha_{3}}, s_{\alpha_{4}}\right]\left(\alpha_{1}, \alpha_{2}, \alpha_{5}, \alpha_{6}\right), \quad P(\mathcal{M})_{\overline{1}}=\left[s_{\alpha_{3}}, s_{\alpha_{4}}\right]\left(\alpha_{2}\right)
$$

with the group of symmetries $\left[s_{\alpha_{3}}, s_{\alpha_{4}}\right]$ which is $D_{\infty}$. The generalized Cartan matrix is

$$
\left(\frac{2\left(\alpha, \alpha^{\prime}\right)}{(\alpha, \alpha)}\right), \quad \alpha, \alpha^{\prime} \in P(\mathcal{M}) .
$$

The automorphic form $B_{\xi_{0,36}^{(2)}}$ coincides with $D_{1 / 2}$ of the weight $1 / 2$ with Fourier expansion

$$
D_{1 / 2}=\frac{1}{2} \sum_{m, n \in \mathbb{Z}}\left(\frac{12}{n}\right)\left(\frac{12}{m}\right) q^{n^{2} / 24} r^{n m / 2} s^{m^{2} / 24} .
$$

See $[41 ;(5.1 .3)]$. 


\section{Appendix: On JaCOBi MOdUlar FORMS WITH INTEGRAL FOURIER COEFFICIENTS}

Here we concentrate on calculational aspects of Jacobi modular forms (or just Jacobi forms) which we use in this paper. We try to avoid complicated subjects related with modular forms and try to be as short as possible. We follow [41], [33], [34].

We shall consider Jacobi forms with respect to Jacobi group $\Gamma^{J}$ which is a semidirect product $\Gamma^{J}=S L_{2}(\mathbb{Z}) \ltimes H(\mathbb{Z})$ where $H(\mathbb{Z})$ is the integral Heisenberg group. The group $H(\mathbb{Z})$ is the central extension of $\mathbb{Z}^{2}$ by $\mathbb{Z}$ which is the center of $\Gamma^{J}$. We denote by $(\lambda, \mu)$ an element of the $\mathbb{Z}^{2}$ and by $\kappa$ an element of $\mathbb{Z}$. The Jacobi group $\Gamma^{J}$ can be identified with $\Gamma_{\infty} /\left\{ \pm E_{4}\right\}$ where $\Gamma_{\infty}$ is a maximal parabolic subgroup of $S p_{4}(\mathbb{Z})$, which consists of all elements preserving a line.

The binary (to $\{ \pm 1\}$ ) character $v_{H}$ on $H(\mathbb{Z})$ which is

$$
v_{H}([\lambda, \mu ; \kappa]):=(-1)^{\lambda+\mu+\lambda \mu+\kappa}
$$

can be extended to a binary character $v_{J}$ of the Jacobi group if one puts $\left.v_{J}\right|_{S L_{2}(\mathbb{Z})}=$ 1.

Definition 3.1. Let $k \in \mathbb{Z} / 2, t \in \mathbb{Z} / 2, t \geq 0$ and $v: S L_{2}(\mathbb{Z}) \rightarrow \mathbb{C}^{*}$ a character (or a multiplier system) of finite order of $S L_{2}(\mathbb{Z})$. A holomorphic function $\phi(\tau, z)$ on $\mathbb{H} \times \mathbb{C}$ (where $\mathbb{H}$ is the upper-half plane $\operatorname{Im} \tau>0$ ) is called a weak Jacobi form of weight $k$ and index $t$ with respect to $\Gamma^{J}$ with the character (or the multiplier system) $v$ if

$$
\phi\left(\frac{a \tau+b}{c \tau+d}, \frac{z}{c \tau+d}\right)=v\left(\left(\begin{array}{ll}
a & b \\
c & d
\end{array}\right)\right)(c \tau+d)^{k} e^{\frac{2 \pi i t c z^{2}}{c \tau+d}} \phi(\tau, z) \quad\left(\left(\begin{array}{ll}
a & b \\
c & d
\end{array}\right) \in S L_{2}(\mathbb{Z})\right),
$$

and

$$
\phi(\tau, z+\lambda \tau+\mu)=(-1)^{2 t(\lambda+\mu)} e^{-2 \pi i t\left(\lambda^{2} \tau+2 \lambda z\right)} \phi(\tau, z) \quad(\lambda, \mu \in \mathbb{Z}),
$$

and it has Fourier expansion of type

$$
\phi(\tau, z)=\sum_{n \geq 0, l \in t+\mathbb{Z}} f(n, l) \exp (2 \pi i(n \tau+l z)) .
$$

We denote the space of all weak Jacobi forms of weight $k$ and index $t$ with $v$ as $J_{k, t}(v)$.

Further we denote $q=\exp (2 \pi i \tau)$ and $r=\exp (2 \pi i z)$, thus $\exp (2 \pi i(n \tau+l z))=$ $q^{n} r^{l}$.

The Dedekind $\eta$-function is

$$
\eta(\tau)=q^{\frac{1}{24}} \prod_{n \geq 1}\left(1-q^{n}\right)=\sum_{n \in \mathbb{N}}\left(\frac{12}{n}\right) q^{n^{2} / 24}
$$

where

$$
\left(\frac{12}{n}\right)=\left\{\begin{aligned}
1 & \text { if } n \equiv \pm 1 \bmod 12 \\
-1 & \text { if } n \equiv \pm 5 \bmod 12
\end{aligned}\right.
$$


The $\eta(\tau)$ is $S L_{2}(\mathbb{Z})$-modular form of the weight $1 / 2$ with some multiplier system $v_{\eta}$ which takes values in 24th roots of unity. The function $\Delta_{12}=\eta^{24}$ (the Ramanujan function) can be considered as a weak Jacobi form $\Delta_{12}(\tau, z)=\Delta_{12}(\tau)$ of weight 12 and index 0, i.e. $\Delta_{12} \in J_{12,0}$. It had been used in (1.3.5). The function $\Delta_{12}$ is equal to zero only at infinity $q=0$.

We remind the classical Eisenstein series

$$
E_{4}(\tau)=1+240 \sum_{n=1}^{\infty} \sigma_{3}(n) q^{n}, \quad E_{6}(\tau)=1-504 \sum_{n=1}^{\infty} \sigma_{5}(n) q^{n},
$$

where $\sigma_{k}(n)=\sum_{m \mid n} m^{k}$. They are $S L_{2}(\mathbb{Z})$-modular forms of the weight 4 and 6 respectively. They give weak Jacobi forms $E_{4} \in J_{4,0}$ and $E_{6} \in J_{6,0}$ of index 0 . We have $\Delta_{12}=\left(E_{4}^{3}-E_{6}^{2}\right) / 1728$ which gives another formula for $\Delta_{12}$. The modular forms $E_{4}, E_{6}$ and $\Delta_{12}$ generate over $\mathbb{Z}$ the ring of holomorphic $S L_{2}(\mathbb{Z})$-modular forms of even weight with integral Fourier coefficients. Over $\mathbb{Q}$ similar ring has two free generators $E_{4}$ and $E_{6}$.

A function $\phi(\tau, z)$ is called a nearly holomorphic Jacobi form if $\Delta^{N} \phi(\tau, z)$ for some $N \geq 0$ is a weak Jacobi form $\left(\Delta^{N} \phi(\tau, z) \in J_{k, t}(v)\right.$ for some $\left.k, t\right)$. Nearly holomorphic Jacobi forms is the most general class of Jacobi forms which we consider here. They may have poles at infinity $q=0$. Their Fourier coefficients $f(n, l)$ depend only on the norm $4 t n-l^{2}$ and $\pm l \bmod 2 t$; the norm $4 t n-l^{2}$ of non-zero Fourier coefficients $f(n, l)$ is bounded from below. A nearly holomorphic Jacobi form is holomorphic at infinity iff its non-zero Fourier coefficients have nonnegative norm. We denote by $J_{k, t}^{n h}$ the space of all nearly holomorphic Jacobi forms of weight $k \in \mathbb{Z} / 2$ and index $t \in \mathbb{Z} / 2, t \geq 0$ with the trivial $S L_{2}(\mathbb{Z})$-character. Respectively, $J_{k, t}$ denotes its subspace of all weak Jacobi forms.

Below we shall describe generators of the ring $J_{0, *}^{\mathbb{Z}}$ of weak Jacobi forms of zero weight, integral index and with integral Fourier coefficients.

The Jacobi theta-series

$$
\begin{aligned}
\vartheta(\tau, z) & =\sum_{n \equiv 1 \bmod 2}(-1)^{\frac{n-1}{2}} \exp \left(\frac{\pi i n^{2}}{4} \tau+\pi i n z\right)=\sum_{m \in \mathbb{Z}}\left(\frac{-4}{m}\right) q^{m^{2} / 8} r^{m / 2} \\
& =-q^{1 / 8} r^{-1 / 2} \prod_{n \geq 1}\left(1-q^{n-1} r\right)\left(1-q^{n} r^{-1}\right)\left(1-q^{n}\right)
\end{aligned}
$$

is a holomorphic Jacobi form of weight $1 / 2$ and index $1 / 2$ with the multiplier system $v_{\eta}^{3}$ where

$$
\left(\frac{-4}{n}\right)=\left\{\begin{array}{cl} 
\pm 1 & \text { if } n \equiv \pm 1 \bmod 4 \\
0 & \text { if }(n, 4) \neq 1
\end{array}\right.
$$

Using $\vartheta(\tau, z)$, we get weak Jacobi forms

$$
\begin{gathered}
\phi_{0, \frac{3}{2}}(\tau, z)=\frac{\vartheta(\tau, 2 z)}{\vartheta(\tau, z)} \\
=r^{-\frac{1}{2}} \prod_{n \geq 1}\left(1+q^{n-1} r\right)\left(1+q^{n} r^{-1}\right)\left(1-q^{2 n-1} r^{2}\right)\left(1-q^{2 n-1} r^{-2}\right) \in J_{0, \frac{3}{2}} \\
\phi_{-1, \frac{1}{2}}(\tau, z)=\frac{\vartheta(\tau, z)}{n(\tau)^{3}}=-r^{-1 / 2} \prod\left(1-q^{n-1} r\right)\left(1-q^{n} r^{-1}\right)\left(1-q^{n}\right)^{-2} \in J_{-1, \frac{1}{2}}
\end{gathered}
$$


and weak Jacobi forms

$$
\begin{gathered}
\phi_{0,3}(\tau, z)=\phi_{0, \frac{3}{2}}(\tau, z)^{2}=\frac{\vartheta(\tau, 2 z)^{2}}{\vartheta(\tau, z)^{2}} \in J_{0,3}, \\
\phi_{-2,1}(\tau, z)=\phi_{-1, \frac{1}{2}}(\tau, z)^{2}=\frac{\vartheta(\tau, z)^{2}}{\eta(\tau)^{6}} \in J_{-2,1} .
\end{gathered}
$$

One can define two other weak Jacobi forms with integral Fourier coefficients

$$
\begin{gathered}
\phi_{0,2}(\tau, z)=\frac{1}{2} \eta(\tau)^{-4} \sum_{m, n \in \mathbb{Z}}(3 m-n)\left(\frac{-4}{m}\right)\left(\frac{12}{n}\right) q^{\frac{3 m^{2}+n^{2}}{24}} r^{\frac{m+n}{2}} \in J_{0,2} \\
\phi_{0,4}(\tau, z)=\frac{\vartheta(\tau, 3 z)}{\vartheta(\tau, z)}=r^{-1} \prod_{m \geq 1}\left(1+q^{m-1} r+q^{2 m-2} r^{2}\right)\left(1+q^{m} r^{-1}+q^{2 m} r^{-2}\right) \\
\times \prod_{n \equiv 1,2 \bmod 3}\left(1-q^{n} r^{3}\right)\left(1-q^{n} r^{-3}\right) \in J_{0,4} .
\end{gathered}
$$

A weak Jacobi form $\phi_{0,1} \in J_{0,1}$ with integral Fourier coefficients is defined by the relation

$$
4 \phi_{0,4}=\phi_{0,1} \phi_{0,3}-\phi_{0,2}^{2}
$$

The Jacobi forms $\phi_{0,1}$ and $\phi_{-2,1}$ were introduced in [24] using different definition. The Jacobi forms $\phi_{0, \frac{1}{2}}, \phi_{-1, \frac{1}{2}}, \phi_{0,2}, \phi_{0,3}, \phi_{0,4}$ were introduced in [41].

By [33], the ring $J_{0, *}^{\mathbb{Z}}$ of all weak Jacobi forms of weight 0 with integral index * and with integral Fourier coefficients is generated over $\mathbb{Z}$ by the weak Jacobi forms $\phi_{0,1}, \phi_{0,2}, \phi_{0,3}$ and $\phi_{0,4}$ with the relation (3.13).

Eisenstein-Jacobi series $E_{4,1} \in J_{4,1}, E_{4,2} \in J_{4,2}, E_{6,1} \in J_{6,1}, E_{6,2} \in J_{6,2}, E_{6,3} \in$ $J_{6,3}$ (see [24] about general results on Eisenstein-Jacobi series) which have integral Fourier coefficients (and the Fourier coefficient 1 for $q^{0} r^{0}$ ) can be found by relations

$$
\begin{gathered}
E_{4} \phi_{0,1}-E_{6} \phi_{-2,1}=12 E_{4,1}, \\
E_{6} \phi_{0,1}-E_{4}^{2} \phi_{-2,1}=12 E_{6,1}, \\
E_{4,1} \phi_{0,1}-E_{6,1} \phi_{-2,1}=12 E_{4,2}, \\
E_{6,1} \phi_{0,1}-E_{4} E_{4,1} \phi_{-2,1}=12 E_{6,2}, \\
E_{4,1} \phi_{0,2}-E_{4} \phi_{0,3}=2 E_{4,3}, \\
E_{6,1} \phi_{0,2}-E_{6} \phi_{0,3}=2 E_{6,3}^{\prime}, \\
E_{6,3}^{\prime}=E_{6,3}+\frac{22}{61} \Delta_{12} \phi_{-2,1}^{3} .
\end{gathered}
$$

By [34], the ring $J_{*, *}^{\mathbb{Z}}$ of weak Jacobi forms of integral weight and integral index with integral Fourier coefficients is generated over $\mathbb{Z}$ by the weak Jacobi forms $E_{4}$, 
proved in $[24]$ that similar ring $J_{*, *}^{\mathbb{Q}}$ over $\mathbb{Q}$ is generated by free generators $E_{4}, E_{6}$, $\phi_{0,1}$ and $\phi_{-2,1}$.

Using $\phi_{0, \frac{3}{2}}(\tau, z) \in J_{0, \frac{3}{2}}$ and $\phi_{-1, \frac{1}{2}}(\tau, z) \in J_{-1, \frac{1}{2}}$, one can get similar results for half-integral index. Using $\Delta_{12}$, one can generalize these results for nearly holomorphic Jacobi forms with integral Fourier coefficients. For example, any nearly holomorphic Jacobi form $\phi_{0, t} \in J_{0, t}^{n h}, t \in \mathbb{N}$, with integral Fourier coefficients needed in Theorem 2.2.1 can be written as

$$
\phi_{0, t}=\frac{P\left(E_{4}, E_{6}, \Delta_{12}, E_{4,1}, E_{4,2}, E_{4,3}, E_{6,1}, E_{6,2}, E_{6,3}^{\prime}, \phi_{0,1}, \phi_{0,2}, \phi_{0,3}, \phi_{0,4}, \phi_{-2,1}\right)}{\Delta_{12}^{N}}
$$

where $P$ is a polynomial with integral coefficients and $N \geq 0$.

\section{REFERENCES}

[1] W.L. Baily, Fourier-Jacobi series, Algebraic groups and discontinuous subgroups. Proc. Symp. Pure Math. Vol. IX (Borel A., Mostow G.D., eds.), Amer. Math. Soc., Providence, Rhode Island, 1966, pp. 296-300.

[2] R. Borcherds, Vertex algebras, Kac-Moody algebras, and the monster, Proc. Natl. Acad. Sci. USA 83 (1986), 3068 - 3071.

[3] R. Borcherds, Generalised Kac-Moody algebras, J. of Algebra 115 (1988), 501-512.

[4] R. Borcherds, The monster Lie algebra, Adv. Math. 83 (1990), 30-47.

[5] R. Borcherds, The monstrous moonshine and monstrous Lie superalgebras, Invent. Math. 109 (1992), 405-444.

[6] R. Borcherds, Sporadic groups and string theory, Proc. European Congress of Mathem. 1992, pp. 411-421.

[7] R. Borcherds, Automorphic forms on $\mathrm{O}_{s+2,2}$ and infinite products, Invent. Math. 120 (1995), 161-213.

[8] R. Borcherds, The moduli space of Enriques surfaces and the fake monster Lie superalgebra, Topology 35 (1996), no. 3, 699-710.

[9] R. Borcherds, Automorphic forms with singularities on Grassmanians, Invent. Math. 132 (1998), no. 3, 491-562; alg-geom/9609022.

[10] R. Borcherds, What is moonshine?, Proc. Int. Congr. Math. Berlin 1998, vol. 1, pp. 607-615; math.QA/9809110.

[11] R. Borcherds, Vertex algebras, Topological field theory, primitive forms and related topics (Kyoto, 1996), Progr. Math. 160., Birkhäuser Boston, Boston, MA, 1998, pp. 35-77; a-alg/9706008.

[12] R. Borcherds, Reflection groups of Lorentzian lattices, Duke Math. J. 104 (2000), no. 2, 319-366; math.GR/9909123.

[13] R. Borcherds, L. Katzarkov, T. Pantev T, N.I. Shepherd-Barron, Families of K3 surfaces, J. Algebraic Geom. 7 (1998), no. 1, 183-193; alg-geom/9701013.

[14] J.H. Bruinier, Borcherdsprodukte und Chernsche Klassen von Hirzebruch-Zagier-Zykeln, Dissertation, Universität Heidelberg (1998).

[15] J.H. Bruinier, Borcherds products and Chern classes of Hirzebruch-Zagier divisors, Invent. Math. 138 (1999), no. 1, $51-83$.

[16] J.H. Bruinier, Borcherds products on $O(2, l)$ and Chern classes of Heegner divisors, Habilitationsschrift, Universität Heidelberg (2000).

[17] G.L. Cardoso, Perturbative gravitational couplings and Siegel modular forms in $D=4, N=2$ string models, Nucl. Phys. Proc. Suppl. 56B (1997), 94-101; hep-th/9612200.

[18] G.L. Cardoso, G. Curio, D. Lust, Perturbative coupling and modular forms in $N=2$ string models with a Wilson line, Nucl. Phys. B491 (1997), 147-183; hep-th/9608154.

[19] J.H. Conway, The automorphism group of the 26 dimensional even Lorentzian lattice, J. Algebra 80 (1983), 159-163.

[20] J.H.Conway, S. Norton, Monstrous moonshine, Bull. London Math. Soc. 11 (1979), 308-339.

[21] R. Dijkgraaf, The mathematics of fivebranes, Proc. Int. Congr. Math. Berlin 1998, vol. 3, 
[22] R. Dijkgraaf, G. Moore, E. Verlinde, H. Verlinde, Elliptic genera of symmetric products and second quantized strings, Commun. Math. Phys. (1997), 197-209; hep-th/9608096.

[23] R. Dijkgraaf, E. Verlinde, H. Verlinde, Counting dyons in $N=4$ string theory, Nucl. Phys. (1997), 543-561; hep-th/9607026.

[24] M. Eichler, D. Zagier, The theory of Jacobi forms, Progress in Math. 55, Birkhäuser, 1985.

[25] I.B. Frenkel, J. Lepowsky, A. Meurmann, Vertex operator algebras and the monster, Academic Press, Boston, MA, 1988.

[26] H. Garland, J. Lepowsky,, Lie algebra homology and the Macdonald-Kac formulas, Invent. Math. 34 (1976), 37-76.

[27] P. Goddard, The work of Richard Ewen Borcherds, Proc. Int. Congr. Math. Berlin 1998, vol. 1, pp. 99-108.

[28] P. Goddard, C.B. Thorn, Compatibility of the dual Pomeron with unitarity and the absence of ghosts in the dual resonance model, Phys. Lett. B40 (1972), no. 2, 235 -238.

[29] V.A. Gritsenko, Jacobi functions of n-variables, Zap. Nauk. Sem. LOMI 168 (1988), 32-45 (Russian); English transl. in J. Soviet Math. 53 (1991), 243-252.

[30] V.A. Gritsenko, Arithmetical lifting and its applications, Number Theory. Proceedings of Paris Seminar 1992-93 (S. David, eds.), Cambridge Univ. Press, 1995, pp. 103-126.

[31] V.A. Gritsenko, Modular forms and moduli spaces of Abelian and K3 surfaces, Algebra i Analyz 6:6 (1994), 65-102 (Russian); English transl. in St.Petersburg Math. Jour. 6:6 (1995), $1179-1208$.

[32] V.A. Gritsenko, Irrationality of the moduli spaces of polarized Abelian surfaces, The International Mathematics Research Notices 6 (1994), 235-243, In full form in "Abelian varieties", Proc. of the Egloffstein conference (1993) de Gruyter, Berlin, 1995, pp. 63-81.

[33] V.A. Gritsenko, Elliptic genus of Calabi-Yau manifolds and Jacobi and Siegel modular forms, St. Petersburg Math. J. 11:5 (1999), 100-125; math.AG/9906190.

[34] V.A. Gritsenko, Complex vector bundles and Jacobi forms, Preprint MPI 76 (1999); math.AG /9906191.

[35] V.A. Gritsenko, K. Hulek, Minimal Siegel modular threefolds, Mathem. Proc. Cambridge Phil. Soc. 123 (1998), 461-485; alg-geom/9506017.

[36] V.A. Gritsenko, V.V. Nikulin, Siegel automorphic form correction of some Lorentzian KacMoody Lie algebras, Amer. J. Math. 119 (1997), no. 1, 181-224; alg-geom/ 9504006.

[37] V.A. Gritsenko, V.V. Nikulin, Siegel automorphic form correction of a Lorentzian KacMoody algebra, C. R. Acad. Sci. Paris Sér. A-B 321 (1995), 1151-1156.

[38] V.A. Gritsenko, V.V. Nikulin, K3 surfaces, Lorentzian Kac-Moody algebras and mirror symmetry, Math. Res. Lett. 3 (1996), no. 2, 211-229; alg-geom/9510008.

[39] V.A. Gritsenko, V.V. Nikulin, The Igusa modular forms and 'the simplest' Lorentzian KacMoody algebras, Mat. Sb. 187 (1996), no. 11, 27-66 (Russian); English transl. in Sb. Math. 187, no. 11, 1601-1641; alg-geom/9603010.

[40] V.A. Gritsenko, V.V. Nikulin, Automorphic forms and Lorentzian Kac-Moody algebras. Part I, Intern. J. Math. 9 (1998), no. 2, 153-199; alg-geom/9610022.

[41] V.A. Gritsenko, V.V. Nikulin, Automorphic forms and Lorentzian Kac-Moody algebras. Part II, Intern. J. Math. 9 (1998), no. 2, 201-275; llg-geom/9611028.

[42] V.A. Gritsenko, V.V. Nikulin, The arithmetic mirror symmetry and Calabi-Yau manifolds, Comm. Math. Phys. 210 (2000), 1-11; alg-geom/9612002.

[43] V.A. Gritsenko, V.V. Nikulin, A lecture about classification of Lorentzian Kac-Moody algebras of the rank three, Preprint Newton Inst. Math. Sci. NI00036-SGT (2000), 1-26; alg-geom/0010329.

[44] V.A. Gritsenko, V.V. Nikulin, On the classification of meromorphic automorphic products and related Lorentzian Kac-Moody algebras with respect to the extended paramodular group (to appear).

[45] J. Harvey, G. Moore, Algebras, BPS-states, and strings, Nucl. Physics. B463 (1996), 315368 ; hep-th/9510182.

[46] J. Igusa, On Siegel modular forms of genus two (II), Amer. J. Math. 84 (1964), no. 2, 392-412.

[47] V. Kac, Infinite dimensional Lie algebras, Cambridge Univ. Press, 1990.

[48] V. Kac, Infinite-dimensional algebras, Dedekind's $\eta$-function, classical Möbius function and 
[49] V. Kac, Vertex algebras for beginners, Univ. Lect. Series (Providence, R.I.). Vol. 10, Amer. Math. Soc., 1998.

[50] V. Kac, M. Wakimoto, Integrable highest weight modules over affine superalgebras and number theory, Lie theory and geometry, Progr. Math., 123, Birkhäuser Boston, Boston, MA, 1994, pp. 415-456.

[51] T. Kawai, $N=2$ heterotic string threshold correction, K3 surfaces and generalized KacMoody superalgebra, Phys. Lett. B371 (1996), 59-64; hep-th/9512046.

[52] T. Kawai, String duality and modular forms, Phys. Lett. B397 (1997), 51-62; hep-th] 9607078 .

[53] T. Kawai, K. Yoshioka, String partition functions and infinite products, Adv. Theor. Math. Phys. 4 (2000), 397-485; hep-th/0002169.

[54] Sh. Kondo, On the Kodaira dimension of the moduli space of K3 surfaces. II, Compositio Math. 116 (1999), no. 2, 111 - 117.

[55] H. Maass, Über ein Analogon zur Vermutung von Saito-Kurokawa, Invent. math. 60 (1980), 85-104.

[56] G. Moore, String duality, automorphic forms and generalised Kac-Moody algebras, Nucl. Phys. Proc. Suppl. 67 (1998), 56-67; hep-th/9710198.

[57] V.V. Nikulin, Integral symmetric bilinear forms and some of their geometric applications, Izv. Akad. Nauk SSSR Ser. Mat. 43 (1979), 111-177 (Russian); English transl. in Math. USSR Izv. 14 (1980).

[58] V.V. Nikulin, On the quotient groups of the automorphism groups of hyperbolic forms by the subgroups generated by 2-reflections, Algebraic-geometric applications, Current Problems in Math. Vsesoyuz. Inst. Nauchn. i Tekhn. Informatsii, Moscow (1981), 3-114 (Russian); English transl. in J. Soviet Math. 22 (1983), 1401-1476.

[59] V.V. Nikulin, On arithmetic groups generated by reflections in Lobachevsky spaces, Izv. Akad. Nauk SSSR Ser. Mat. 44 (1980), 637-669 (Russian); English transl. in Math. USSR Izv. 16 (1981).

[60] V.V. Nikulin, On the classification of arithmetic groups generated by reflections in Lobachevsky spaces, Izv. Akad. Nauk SSSR Ser. Mat. 45 (1981), no. 1, 113-142 (Russian); English transl. in Math. USSR Izv. 18 (1982).

[61] V.V. Nikulin, Discrete reflection groups in Lobachevsky spaces and algebraic surfaces, Proc. Int. Congr. Math. Berkeley 1986, vol. 1, pp. 654-669.

[62] V.V. Nikulin, A lecture on Kac-Moody Lie algebras of the arithmetic type, Preprint Queen's University, Canada \#1994-16, 1994 ; alg-geom/9412003.

[63] V.V. Nikulin, Basis of the diagram method for generalized reflection groups in Lobachevsky spaces and algebraic surfaces with nef anticanonical class, Int. J. Mathem. 7 (1996), no. 1, $71-108$.

[64] V.V. Nikulin, Reflection groups in Lobachevsky spaces and the denominator identity for Lorentzian Kac-Moody algebras, Izv. Akad. Nauk of Russia. Ser. Mat. 60 (1996), no. 2, 73-106 (Russian); English transl. in Russian Acad. Sci. Izv. Math. ; alg-geom/9503003.

[65] V.V. Nikulin, The remark on discriminants of K3 surfaces moduli as sets of zeros of automorphic forms, J. Math. Sci., New York 81 (1996), no. 3, 2738-2743; alg-geom/9512018.

[66] V.V. Nikulin, K3 surfaces with interesting groups of automorphisms, J. Math. Sci., New York 95 (1999), no. 1, 2028-2048; alg-geom/9701011.

[67] V.V. Nikulin, A theory of Lorentzian Kac-Moody algebras, Trudy Mezhdunar. konf. posvyashch. 90-letiyu so dnya rozhdeniya L.S. Pontryagina, T.8: Algebra (Proc. Int. Conf. Devoted to the 90-th Anniversary of L.S. Pontryagin, vol. 8: Algebra), VINITI (Itogi nauki i tekhniki. Sovremennaya matematika i ee prilozheniya. Tematicheskie obzory, vol. 69), Moscow, 1999, pp. 148-167 (Russian); math.AG/9810001.

[68] V.V. Nikulin, On the classification of hyperbolic root systems of the rank three, Trudy Matem. Instit. V.A. Steklov, T. 230, Moscow, 2000, pp. 255 (Russian); English translation in Proc. Steklov Math. Institute. Vol. 230, Moscow, 2000; alg-geom/9711032; alg-geom/9712033; math.AG/9905150.

[69] U. Ray, A character formula for generalised Kac-Moody superalgebras, J. of Algebra 177 (1995), 154-163.

[70] U. Ray, Generalized Kac-Moody algebras and some related topics, Bull. Amer. Math. Soc. 
[71] É.B. Vinberg, The absence of crystallographic reflection groups in Lobachevsky spaces of large dimension, Trudy Moscow. Mat. Obshch. 47 (1984), 68 - 102 (Russian); English transl. in Trans. Moscow Math. Soc. 47 (1985).

[72] É.B. Vinberg, Hyperbolic reflection groups, Uspekhi Mat. Nauk 40 (1985), 29-66 (Russian); English transl. in Russian Math. Surveys 40 (1985).

[73] É.B. Vinberg, Discrete reflection groups in Lobachevsky spaces, Proc. Int. Congr. Math. Warsaw, 1983, vol. 1, 2, pp. 593-601.

University Lille 1, UfR de Mathematiques, F-59655 Villeneuve D'AscQ Cedex, FRANCE

POMi, St. Petersburg, Russia

E-mail address: Valery.Gritsenko@agat.univ-lille1.fr

Deptm. of Pure Mathem. The University of Liverpool, Liverpool L69 3BX, UK; Steklov Mathematical Institute, ul. Gubkina 8, Moscow 117966, GSP-1, Russia E-mail address: vnikulin@liv.ac.uk slava@nikulin.mian.su 